\title{
EXTENSION PROPERTY OF SEMIPOSITIVE INVERTIBLE SHEAVES OVER A NON-ARCHIMEDEAN FIELD
}

\author{
HUAYI CHEN AND ATSUSHI MORIWAKI
}

\begin{abstract}
АвstRAct. In this article, we prove an extension property of semipositively metrized ample invertible sheaves on a projective scheme over a complete non-archimedean valued field. As an application, we establish a Nakai-Moishezon type criterion for adelically normed graded linear series.
\end{abstract}

\section{INTRODUCTION}

Let $k$ be a field and $X$ be a projective scheme over Speck, equipped with an ample invertible $\mathscr{O}_{X}$-module $L$. If $Y$ is a closed subscheme of $X$, then for sufficiently positive integer $n$, any section $\ell$ of $\left.L\right|_{Y} ^{\otimes n}$ on $Y$ extends to a global section of $L^{\otimes n}$ on $X$. In other words, the restriction map $H^{0}\left(X, L^{\otimes n}\right) \rightarrow H^{0}\left(Y,\left.L\right|_{Y} ^{\otimes n}\right)$ is surjective. A simple proof of this result relies on Serre's vanishing theorem, which ensures that $H^{1}\left(X, \mathcal{I}_{Y} \otimes L^{\otimes n}\right)=0$ for sufficiently positive integer $n$, where $\mathcal{I}_{Y}$ is the ideal sheaf of $Y$.

The metrized version (with $k=\mathbb{C}$ ) of this result has been widely studied in the literature and has divers applications in complex analytic geometry and in arithmetic geometry. We assume that the ample invertible sheaf $L$ is equipped with a continuous (with respect to the analytic topology) metric $|\cdot|_{h}$, which induces a continuous metric $|\cdot|_{h^{n}}$ on each tensor power sheaf $L^{\otimes n}$, where $n \in \mathbb{N}, n \geqslant 1$. The metric $|\cdot|_{h^{n}}$ leads to a supremum norm $\|\cdot\|_{h^{n}}$ on the global section space $H^{0}(X, L)$ such that

$$
\forall s \in H^{0}(X, L),\|s\|_{h^{n}}=\sup _{x \in X(\mathbb{C})}|s|_{h^{n}}(x) .
$$

Similarly, it induces a supremum norm $\|\cdot\|_{Y, h^{n}}$ on the space $H^{0}\left(Y,\left.L\right|_{Y} ^{\otimes n}\right)$ with

$$
\|s\|_{Y, h^{n}}=\sup _{y \in Y(\mathbb{C})}|s|_{h^{n}}(y) .
$$

Note that for any section $s \in H^{0}\left(X, L^{\otimes n}\right)$ one has $\left\|\left.s\right|_{Y}\right\|_{Y, h^{n}} \leqslant\|s\|_{h^{n}}$. The metric extension problem consists of studying the extension of global sections of $\left.L\right|_{Y}$ to those of $L$ with an estimation on the supremum norms. Note that a positivity condition on the metric $h$ is in general necessary to obtain interesting upper bounds. This problem has been studied by using Hörmander's $L^{2}$ estimates (see [8] for example), under smoothness conditions on the metric. More recently, it has been proved (without any regularity condition) that, if the metric $|\cdot|_{h}$ is semi-positive, then for any $\epsilon>0$ and any section $l \in H^{0}\left(Y,\left.L\right|_{Y}\right)$ there exist an integer $n \geqslant 1$

Date: 23 November, 2015 (Version 1.5).

2010 Mathematics Subject Classification. Primary 14C20; Secondary 14G40. 
and $s \in H^{0}\left(X, L^{\otimes n}\right)$ such that $\left.s\right|_{Y}=l^{\otimes n}$ and that $\|s\|_{h^{n}} \leqslant e^{\epsilon n}\left\|\left.s\right|_{Y}\right\|_{Y, h^{n}}$. We refer the readers to [18, 16] for more details.

The purpose of this article is to study the non-archimedean counterpart of the above problem. We will establish the following result (see Theorem 4.2 and Corollary 1.2).

Theorem 0.1. Let $k$ be a field equipped with a complete and non-archimedean absolute value $|\cdot|$ (which could be trivial). Let X be a projective scheme over Speck and $L$ be an ample invertible sheaf on $X$, equipped with a continuous and semi-positive metric $|\cdot|_{h}$. Let $Y$ be a closed subscheme of $X$ and $l \in H^{0}\left(Y,\left.L\right|_{Y}\right)$. For any $\epsilon>0$ there exists an integer $n_{0} \geq 1$ such that, for any integer $n \geq n_{0}$, the section $l \otimes n$ extends to a section $s \in H^{0}\left(X, L^{\otimes n}\right)$ verifying $\|s\|_{h} \leq e^{\epsilon n}\|l\|_{Y, h}^{n}$.

The semi-positivity condition of the metric means that the metric $|\cdot|_{h}$ can be written as a uniform limit of Fubini-Study metrics. We will show that, if the absolute value $|\cdot|$ is non-trivial, then this condition is equivalent to the classical semi-positivity condition (namely uniform limit of nef model metrics, see Proposition 3.17) of Zhang [20], see also [9, 15], and compare with the complex analytic case [19]. The advantage of the new definition is that it also works in the trivial valuation case, where the model metrics are too restrictive. We use an argument of extension of scalars to the ring of formal Laurent series to obtain the result of the above theorem in the trivial valuation case.

As an application, we establish an adelic version of the arithmetic NakaiMoishezon criterion as follows, see Theorem 5.5 and Corollary 5.6 infra.

Theorem 0.2. Let $X$ be a geometrically integral projective scheme over a number field $K$ and $L$ be an invertible sheaf on $X$. For any place $v$ of $K$, let $h_{v}$ be a continuous semipositive metric on the pull-back of $L$ on the analytic space $X_{v}^{\text {an }}$, such that $\left(H^{0}\left(X, L^{\otimes n}\right),\left\{\|\cdot\|_{X_{v},}, h_{v}^{n}\right\}\right)$ forms an adelically normed vector space over $K$ for any $n \in \mathbb{N}$ (see Definition [5.1]. Suppose that for any integral closed subscheme $Y$ of $X$, the restriction of $L$ on $Y$ is big and there exist a positive integer $n$ and a non-zero section $s \in H^{0}\left(Y,\left.L\right|_{Y} ^{\otimes n}\right)$ such that $\|s\|_{Y_{v}, h_{v}^{n}} \leqslant 1$ for any place $v$ of $K$, and that the inequality is strict when $v$ is an infinite place. Then for sufficiently positive integer $n$, the Q-vector space $H^{0}\left(X, L^{\otimes n}\right)$ has a basis $\left(\omega_{1}, \ldots, \omega_{r_{n}}\right)$ with $\left\|\omega_{i}\right\|_{X_{v}, h_{v}^{n}} \leqslant 1$ for any place $v$, where the inequality is strict if $v$ is an infinite place.

This result generalizes simultaneously [20, Theorem 4.2] and [14, Theorem 4.2] since here we have a weaker assumption on the adelic metric on $L$. The main idea is to combine the estimation on normed Noetherian graded linear series developed in [14] and the non-archimedean extension property established in the current paper. In the archimedean case we also use the archimedean extension property proved in [16].

The article is organized as follows. In the first section we introduce the notation of the article and prove some preliminary results, most of which concern finite dimensional normed vector spaces over a non-archimedean field. In the second section, we discuss some property of model metrics. In the third section, we study various properties of continuous metrics on an invertible sheaf, where an emphasis is made on the positivity of such metrics. In the fourth section, we prove the extension theorem. Finally, in the fifth and last section, we apply the extension property to prove a generalized arithmetic Nakai-Moishezon's criterion. 


\section{NotATION AND PRELIMINARIES}

1.1. Notation. Throughout this paper, we fix the following notation.

1.1.1. Fix a field $k$ with a non-archimedean absolute value $|\cdot|$. Unless otherwise noted, we assume that $k$ is complete. The valuation ring of $k$ and the maximal ideal of the valuation ring are denoted by $\mathfrak{o}_{k}$ and $\mathfrak{m}_{k}$, respectively, that is,

$$
\mathfrak{o}_{k}:=\{a \in k|| a \mid \leq 1\} \quad \text { and } \quad \mathfrak{m}_{k}:=\{x \in k|| x \mid<1\} .
$$

In the case where $|\cdot|$ is discrete, we fix a uniformizing parameter $\mathscr{\omega}$ of $\mathfrak{m}_{k}$, that is, $\mathfrak{m}_{k}=\omega^{\mathfrak{o}_{k}}$.

1.1.2. A norm $\|\cdot\|$ of a finite-dimensional vector space $V$ over the non-archimedean field $k$ is always assumed to be ultrametric, that is, $\|x+y\| \leq \max \{\|x\|,\|y\|\}$. A pair $(V,\|\cdot\|)$ is called a normed finite-dimensional vector space over $k$.

1.1.3. In Section $1 \sim$ Section 4 , we fix an algebraic scheme $X$ over Speck, that is, $X$ is a scheme of finite type over $\operatorname{Spec}(k)$. Let $X^{\text {an }}$ be the analytification of $X$ in the sense of Berkovich [2]. For $x \in X^{\text {an }}$, the residue field of the associated scheme point of $x$ is denoted by $\kappa(x)$. Note that the seminorm $|\cdot|_{x}$ at $x$ yields an absolute value of $\kappa(x)$. By abuse of notation, it is denoted by $|\cdot|_{x}$. Let $\hat{\kappa}(x)$ be the completion of $\kappa(x)$ with respect to $|\cdot|_{x}$. The extension of $|\cdot|_{x}$ to $\hat{\kappa}(x)$ is also denoted by the same symbol $|\cdot|_{x}$. The valuation ring of $\hat{\kappa}(x)$ and the maximal ideal of the valuation ring are denoted by $\mathfrak{o}_{x}$ and $\mathfrak{m}_{x}$, respectively. Let $L$ be an invertible sheaf on $X$. For $x \in X^{\text {an }}, L \otimes_{\mathscr{O}_{X}} \hat{\kappa}(x)$ is denoted by $L(x)$.

1.1.4. By continuous metric on $L$, we refer to a family $h=\left\{|\cdot|_{h}(x)\right\}_{x \in X}$ an, where $|\cdot|_{h}(x)$ is a norm on $L \otimes_{\mathscr{O}_{X}} \hat{\kappa}(x)$ over $\hat{\kappa}(x)$ for each $x \in X^{\text {an }}$, such that for any local basis $\omega$ of $L$ over a Zariski open subset $U,|\omega|_{h}(\cdot)$ is a continuous function on $U^{\text {an }}$. We assume that $X$ is projective. Given a continuous metric $h$ on $L$, we define a norm $\|\cdot\|_{h}$ on $H^{0}(X, L)$ such that

$$
\forall s \in H^{0}(X, L), \quad\|s\|_{h}:=\sup _{x \in X^{\text {an }}}|s|_{h}(x) .
$$

Similarly, if $Y$ is a closed subscheme of $X$, we define a norm $\|\cdot\|_{Y, h}$ on $H^{0}(Y, L)$ such that

$$
\forall l \in H^{0}(Y, L), \quad\|l\|_{Y, h}:=\sup _{y \in Y_{\text {an }}}|l|_{h}(y) .
$$

Clearly one has

$$
\|s\|_{h} \geqslant\left\|\left.s\right|_{Y}\right\|_{Y, h}
$$

for any $s \in H^{0}(X, L)$.

- In the following 1.1.5, 1.1.6 and 1.1.7 $X$ is always assumed to be projective. 
1.1.5. Given a continuous metric $h$ on $L$, the metric induces for each integer $n \geqslant 1$ a continuous metric on $L^{\otimes n}$ which we denote by $h^{n}$ : for any point $x \in X^{\text {an }}$ and any local basis $\omega$ of $L$ over a Zariski open neighborhood of $x$ one has

$$
\left|\omega^{\otimes n}\right|_{h^{n}}(x)=|\omega|_{h}(x)^{n} .
$$

Note that for any section $s \in H^{0}(X, L)$ one has $\left\|s^{\otimes n}\right\|_{h^{n}}=\|s\|_{h}^{n}$. By convention, $h^{0}$ denotes the trivial metric on $L^{\otimes 0}=\mathscr{O}_{X}$, namely $|\mathbf{1}|_{h^{0}}(x)=1$ for any $x \in X^{\text {an }}$, where 1 denotes the section of unity of $\mathscr{O}_{X}$.

Conversely, given a continuous metric $g=\left\{|\cdot|_{g}(x)\right\}_{x \in X^{\text {an }}}$ on $L^{\otimes n}$, there is a unique continuous metric $h$ on $L$ such that $h^{n}=g$. We denote by $g^{1 / n}$ this metric. This observation allows to define continuous metrics on an element in $\operatorname{Pic}(X) \otimes \mathbb{Q}$ as follows. Given $M \in \operatorname{Pic}(X) \otimes \mathbb{Q}$, we denote by $\Gamma(M)$ the subsemigroup of $\mathbb{N}_{\geq 1}$ of all positive integers $n$ such that $M^{\otimes n} \in \operatorname{Pic}(X)$. We call continuous metric on $M$ any family $g=\left(g_{n}\right)_{n \in \Gamma(M)}$ with $g_{n}$ being a continuous metric on $M^{\otimes n}$, such that $g_{n}^{m}=g_{m n}$ for any $n \in \Gamma(M)$ and any $m \in \mathbb{N}_{\geq 1}$. Note that the family $g=\left(g_{n}\right)_{n \in \Gamma(M)}$ is uniquely determined by any of its elements. In fact, given an element $n \in \Gamma(M)$, one has $g_{m}=g_{m n}^{1 / n}=\left(g_{n}^{m}\right)^{1 / n}$ for any $m \in \Gamma(M)$. In particular, for any positive rational number $p / q$, the family $g^{p / q}=\left(g_{N n p}^{1 / N q}\right)_{n \in \Gamma\left(M^{\otimes(p / q)}\right)}$ is a continuous metric on $M^{\otimes(p / q)}$, where $N$ is a positive integer such that $M^{\otimes N} \in$ $\operatorname{Pic}(X)$, and the metric $g^{p / q}$ does not depend on the choice of the positive integer $N$. If $L$ is an element of $\operatorname{Pic}(X)$, equipped with a continuous metric $g$. By abuse of notation, we use the expression $g$ to denote the metric family $\left(g^{n}\right)_{n \in \mathbb{N} \geqslant 1}$, viewed as a continuous metric on the canonical image of $L$ in $\operatorname{Pic}(X) \otimes \mathbb{Q}$.

Let $M$ be an element in $\operatorname{Pic}(X) \otimes \mathbb{Q}$ equipped with a continuous metric $g=$ $\left(g_{n}\right)_{n \in \Gamma(M)}$. By abuse of notation, for $n \in \Gamma(M)$ we also use the expression $g^{n}$ to denote the continuous metric $g_{n}$ on $M^{\otimes n}$.

1.1.6. Let $\mathscr{X} \rightarrow \operatorname{Spec}\left(\mathfrak{o}_{k}\right)$ be a projective and flat $\mathfrak{o}_{k}$-scheme such that the generic fiber of $\mathscr{X} \rightarrow \operatorname{Spec}\left(\mathfrak{o}_{k}\right)$ is $X$. We call it a model of $X$. We denote by $\mathscr{X}_{0}:=$ $\mathscr{X} \otimes_{\mathfrak{o}_{k}}\left(\mathfrak{o}_{k} / \mathfrak{m}_{k}\right)$ the central fiber of $\mathscr{X} \rightarrow \operatorname{Spec}\left(\mathfrak{o}_{k}\right)$. By the valuative criterion of properness, for any point $x \in X^{\text {an }}$, the canonical $k$-morphism Spec $\hat{\kappa}(x) \rightarrow X$ extends in a unique way to an $\mathfrak{o}_{k}$-morphism of schemes $\mathscr{P}_{x}:$ Spec $\mathfrak{o}_{x} \rightarrow \mathscr{X}$. We denote by $r_{\mathscr{X}}(x)$ the image of $\mathfrak{m}_{x} \in$ Spec $\mathfrak{o}_{x}$ by the map $\mathscr{P}_{x}$. Thus we obtain a map $r_{\mathscr{X}}$ from $X^{\text {an }}$ to $\mathscr{X}_{0}$, called the reduction map of $\mathscr{X}$.

Let $\mathscr{L}$ be an element of $\operatorname{Pic}(\mathscr{X}) \otimes \mathbb{Q}$ such that $\left.\mathscr{L}\right|_{X}=L$ in $\operatorname{Pic}(X) \otimes \mathbb{Q}$. The Q-invertible sheaf $\mathscr{L}$ yields a continuous metric $|\cdot| \mathscr{L}$ as follows.

First we assume that $\mathscr{L} \in \operatorname{Pic}(\mathscr{X})$ and $\left.\mathscr{L}\right|_{X}=L$ in $\operatorname{Pic}(X)$. For any $x \in X^{\text {an }}$, let $\omega_{x}$ be a local basis of $\mathscr{L}$ around $r_{\mathscr{X}}(x)$ and $\bar{\omega}_{x}$ the class of $\omega_{x}$ in $L(x):=$ $L \otimes_{\mathscr{O}_{X}} \hat{\kappa}(x)$. For $l \in L \otimes_{\mathscr{O}_{X}} \hat{\kappa}(x)$, if we set $l=a_{x} \bar{\omega}_{x}\left(a_{x} \in \hat{\kappa}(x)\right)$, then $|l|_{\mathscr{L}}(x):=$ $\left|a_{x}\right|_{x}$. Here we set $h:=\{|\cdot| \mathscr{L}(x)\}_{x \in X^{\text {an }}}$. Note that $h$ is continuous because, for a local basis $\omega$ of $\mathscr{L}$ over an open set $\mathscr{U}$ of $\mathscr{X},|\omega|_{\mathscr{L}}(x)=1$ for all $x \in r_{\mathscr{X}}^{-1}\left(\mathscr{U}_{\circ}\right)$. Moreover,

$$
|\cdot|_{h^{n}}(x)=|\cdot|_{\mathscr{L}^{n}}(x)
$$

for all $n \geq 0$ and $x \in X^{\text {an }}$. Indeed, if we set $l=a_{x} \bar{\omega}_{x}$ for $l \in L(x)$, then $l^{\otimes n}=a_{x}^{n} \bar{\omega}_{x}^{\otimes n}$. Thus

$$
\left|l^{\otimes n}\right|_{h^{n}}(x)=\left(|l|_{h}(x)\right)^{n}=\left|a_{x}\right|_{x}^{n}=\left|l^{\otimes n}\right|_{\mathscr{L}^{n}}(x) .
$$


In general, there are $\mathscr{M} \in \operatorname{Pic}(\mathscr{X})$ and a positive integer $m$ such that $\mathscr{L}^{\otimes m}=$ $\mathscr{M}$ in $\operatorname{Pic}(\mathscr{X}) \otimes \mathbb{Q}$ and $\left.\mathscr{M}\right|_{X}=L^{\otimes m}$ in $\operatorname{Pic}(X)$. Then

$$
|\cdot|_{\mathscr{L}}(x):=\left(|\cdot|_{\mathscr{M}}(x)\right)^{1 / m} \text {. }
$$

Note that the above definition does not depend on the choice of $\mathscr{M}$ and $m$. Indeed, let $\mathscr{M}^{\prime}$ and $m^{\prime}$ be another choice. As $\mathscr{M}^{\otimes m^{\prime}}=\mathscr{M}^{\prime \otimes m}$ in $\operatorname{Pic}(\mathscr{X}) \otimes \mathbb{Q}$, there is a positive integer $N$ such that $\mathscr{M}^{\otimes N m^{\prime}}=\mathscr{M}^{\otimes}{ }^{\otimes m}$ in $\operatorname{Pic}(\mathscr{X})$, so that, by using (1.2),

$$
\left(|\cdot|_{\mathscr{M}}(x)\right)^{N m^{\prime}}=|\cdot|_{\mathscr{M}^{\otimes N m^{\prime}}}(x)=|\cdot|_{\mathscr{M}^{\prime \otimes N m}}(x)=\left(|\cdot|_{\mathscr{M}^{\prime}}(x)\right)^{N m},
$$

as desired.

1.1.7. Let $\mathscr{X}$ be a model of $X$. As $\mathscr{X}$ is flat over $\mathfrak{o}_{k}$, the natural homomorphism $\mathscr{O}_{\mathscr{X}} \rightarrow \mathscr{O}_{X}$ is injective. Let $Y$ be a closed subscheme of $X$ and $I_{Y} \subseteq \mathscr{O}_{X}$ the defining ideal sheaf of $Y$. Let $\mathscr{I}_{\mathscr{Y}}$ be the kernel of $\mathscr{O}_{\mathscr{X}} \rightarrow \mathscr{O}_{X} / I_{Y}$, that is, $\mathscr{I}_{\mathscr{Y}}:=$ $\mathscr{I}_{Y} \cap \mathscr{O}_{\mathscr{X}}$. Obviously $\mathscr{I}_{\mathscr{Y}} \otimes_{\mathfrak{o}_{k}} k=I_{Y}$, so that if we set $\mathscr{Y}=\operatorname{Spec}\left(\mathscr{O}_{\mathscr{X}} / \mathscr{I}_{\mathscr{Y}}\right)$, then $\mathscr{Y} \times \operatorname{Spec}\left(\mathfrak{o}_{k}\right) \operatorname{Spec}(k)=Y$. Moreover, $\mathscr{Y}$ is flat over $\mathfrak{o}_{k}$ because $\mathscr{O}_{\mathscr{Y}} \rightarrow \mathscr{O}_{Y}$ is injective. Therefore, $\mathscr{Y}$ is a model of $Y$. We say that $\mathscr{Y}$ is the Zariski closure of $Y$ in $\mathscr{X}$.

1.2. Extension obstruction index. In this subsection, we introduce an invariant to describe the obstruction to the extension property. Let $X$ be a projective scheme over Spec $k, L$ be an invertible sheaf on $X$ equipped with a continuous metric $h$, and $Y$ be a closed subscheme of $X$. For any non-zero element $l$ of $H^{0}\left(Y,\left.L\right|_{Y}\right)$, we denote by $\lambda_{h}(l)$ the following number (if there does not exist any section $s \in H^{0}\left(X, L^{\otimes n}\right)$ extending $l^{\otimes n}$, then the infimum in the formula is defined to be $+\infty$ by convention)

$$
\lambda_{h}(l)=\limsup _{n \rightarrow+\infty} \inf _{\substack{\left.s \in H^{0}\left(X, L^{\otimes n}\right) \\ s\right|_{Y}=l^{\otimes n}}}\left(\frac{\log \|s\|_{h^{n}}}{n}-\log \|l\|_{Y, h}\right) \in[0,+\infty] .
$$

This invariant allows to describe in a numerically way the obstruction to the metric extendability of the section $l$. In fact, the following assertions are equivalent:

(a) $\lambda_{h}(l)=0$,

(b) for any $\epsilon>0$, there exists $n_{0} \in \mathbb{N}_{\geq 1}$ such that, for any integer $n \geq n_{0}$, the element $l^{\otimes n}$ extends to a section $s \in H^{0}\left(X, L^{\otimes n}\right)$ such that $\|s\|_{h} \leq e^{\epsilon n}\|l\|_{Y, h}^{n}$.

The following proposition shows that, if $l^{\otimes n}$ extends to a global section of $L^{\otimes n}$ for sufficiently positive $n$ (it is the case notably when the line bundle $L$ is ample), then the limsup defining $\lambda_{h}(l)$ is actually a limit.

Proposition 1.1. For any integer $n \geqslant 1$, let

$$
a_{n}=\inf _{\substack{\left.s \in H^{0}\left(X, L^{\otimes n}\right) \\ s\right|_{Y}=l \otimes n}}\left(\log \|s\|_{h^{n}}-n \log \|l\|_{Y, h}\right) .
$$

Then the sequence $\left(a_{n}\right)_{n \geq 1}$ is sub-additive, namely one has $a_{m+n} \leq a_{m}+a_{n}$ for any $(m, n) \in \mathbb{N}_{\geq 1}$. In particular, if for sufficiently positive integer $n$, the section $l^{n}$ lies in the image of the restriction map $H^{0}\left(X, L^{\otimes n}\right) \rightarrow H^{0}\left(Y,\left.L\right|_{Y} ^{\otimes n}\right)$, then "lim sup" in (1.3) can actually be replaced by "lim". 
Proof. By (1.1), one has $a_{n} \geq 0$ for any integer $n \geq 1$. Moreover, $a_{n}<+\infty$ if and only if $l^{n}$ lies in the image of the restriction map $H^{0}\left(X, L^{\otimes n}\right) \rightarrow H^{0}\left(Y,\left.L\right|_{Y} ^{\otimes n}\right)$. To verify the inequality $a_{m+n} \leq a_{m}+a_{n}$, it suffices to consider the case where both $a_{m}$ and $a_{n}$ are finite. Let $s_{m}$ and $s_{n}$ be respectively sections in $H^{0}\left(X, L^{\otimes m}\right)$ and $H^{0}\left(X, L^{\otimes n}\right)$ such that $\left.s_{m}\right|_{Y}=l^{\otimes m}$ and $\left.s_{n}\right|_{Y}=l^{\otimes n}$, then the section $s=s_{m} \otimes s_{n} \in$ $H^{0}\left(X, L^{\otimes(m+n)}\right)$ verifies the relation $\left.s\right|_{Y}=l^{\otimes(n+m)}$. Moreover, one has

$$
\|s\|_{h}=\sup _{x \in X^{\mathrm{an}}}|s|_{h}(x)=\sup _{x \in X^{\mathrm{an}}}\left|s_{m}\right|_{h}(x) \cdot\left|s_{n}\right|_{h}(x) \leqslant\left\|s_{m}\right\|_{h} \cdot\left\|s_{n}\right\|_{h} .
$$

Since $s_{m}$ and $s_{n}$ are arbitrary, one has $a_{m+n} \leq a_{m}+a_{n}$. Finally, by Fekete's lemma, if $a_{n}<+\infty$ for sufficiently positive integer $n$, then the sequence $\left(a_{n} / n\right)_{n \geq 1}$ actually converges in $\mathbb{R}_{+}$. The proposition is thus proved.

Corollary 1.2. Assume that the invertible sheaf $L$ is ample, then the following conditions are equivalent.

(a) $\lambda_{h}(l)=0$,

(b) for any $\epsilon>0$, there exists $n \in \mathbb{N}_{\geq 1}$ and a section $s \in H^{0}\left(X, L^{\otimes n}\right)$ such that $\left.s\right|_{Y}=l^{n}$ and that $\|s\|_{h} \leq e^{\epsilon n}\|l\|_{Y, h}$.

Proof. We keep the notation of the previous proposition. By definition the second condition is equivalent to

$$
\liminf _{n \rightarrow+\infty} \frac{a_{n}}{n}=0 .
$$

Since $L$ is ample, Proposition 1.1 leads to the convergence of the sequence $\left(a_{n} / n\right)_{n \geq 1}$ in $\mathbb{R}_{+}$. Hence the condition (1.4) is equivalent to $\lambda_{h}(l)=0$.

1.3. Normed vector space over a non-archimedean field. In this subsection, we recall several facts on (ultrametric) norms over a non-archimedean field. Throughout this subsection, a norm on a vector space over a non-archimedean field is always assumed to be ultrametric. We also assume that $k$ is complete except in \$1.3.1.

1.3.1. Orthogonality of bases. In this subsubsection, $k$ is not necessarily complete. Let $V$ be a finite-dimensional vector space over $k$ and $\|\cdot\|$ a norm of $V$ over $(k,|\cdot|)$. Let $r$ be the rank of $V$. We assume that $\|\cdot\|$ extends to a norm on $V \otimes_{k} \widehat{k}$, where $\widehat{k}$ denotes the completion of $(k,|\cdot|)$, on which the absolute value extends in a unique way. In particular, any $k$-linear isomorphism $k^{r} \rightarrow V$ is a homeomorphism, where we consider the product topology on $k^{r}$ (see [4] §I.2, no.3, Theorem 2 and the remark on the page I.15), and any vector subspace of $V$ is closed.

For a basis $\boldsymbol{e}=\left(e_{1}, \ldots, e_{r}\right)$ of $V$, we set

$$
\forall\left(a_{1}, \ldots, a_{r}\right) \in \widehat{k}^{r}, \quad\left\|a_{1} e_{1}+\cdots+a_{r} e_{r}\right\|_{e}:=\max \left\{\left|a_{1}\right|, \ldots,\left|a_{r}\right|\right\},
$$

which yields an ultrametric norm on $V \otimes_{k} \widehat{k}$. Note that the norms $\|\cdot\|_{e}$ and $\|\cdot\|$ on $V$ are equivalent.

For $\alpha \in(0,1]$, a basis $\left(e_{1}, \ldots, e_{r}\right)$ of $V$ is called an $\alpha$-orthogonal basis of $V$ with respect to $\|\cdot\|$ if

$$
\alpha \max \left\{\left|a_{1}\right|\left\|e_{1}\right\|, \ldots,\left|a_{r}\right|\left\|e_{r}\right\|\right\} \leq\left\|a_{1} e_{1}+\cdots+a_{r} e_{r}\right\| \quad\left(\forall a_{1}, \ldots, a_{r} \in k\right) .
$$

If $\alpha=1$ (resp. $\alpha=1$ and $\left\|e_{1}\right\|=\cdots=\left\|e_{r}\right\|=1$ ), then the above basis is called an orthogonal basis of $V$ (resp. an orthonormal basis of $V$ ). We refer the readers to 
[17. §2.3] for more details on the orthogonality in the non-archimedean setting. Let $\left(e_{1}^{\prime}, \ldots, e_{r}^{\prime}\right)$ be another basis of $V$. We say that $\left(e_{1}, \ldots, e_{r}\right)$ is compatible with $\left(e_{1}^{\prime}, \ldots, e_{r}^{\prime}\right)$ if $k e_{1}+\cdots+k e_{i}=k e_{1}^{\prime}+\cdots+k e_{i}^{\prime}$ for $i=1, \ldots, r$.

Proposition 1.3. Fix a basis $\left(e_{1}^{\prime}, \ldots, e_{r}^{\prime}\right)$ of $V$. For any $\alpha \in(0,1)$, there exists an $\alpha$ orthogonal basis $\left(e_{1}, \ldots, e_{r}\right)$ of $V$ with respect to $\|\cdot\|$ such that $\left(e_{1}, \ldots, e_{r}\right)$ is compatible with $\left(e_{1}^{\prime}, \ldots, e_{r}^{\prime}\right)$. Moreover, if the absolute value $|\cdot|$ is discrete, then there exists an orthogonal basis $\left(e_{1}, \ldots, e_{r}\right)$ of $V$ compatible with $\left(e_{1}^{\prime}, \ldots, e_{r}^{\prime}\right)$ (cf. [6. Proposition 2.5]).

Proof. We prove it by induction on $\operatorname{dim}_{k} V$. If $\operatorname{dim}_{k} V=1$, then the assertion is obvious. By the hypothesis of induction, there is a $\sqrt{\alpha}$-orthogonal basis $\left(e_{1}, \ldots, e_{r-1}\right)$ of $V^{\prime}:=k e_{1}^{\prime}+\cdots+k e_{r-1}^{\prime}$ with respect to $\|\cdot\|$ such that

$$
k e_{1}+\cdots+k e_{i}=k e_{1}^{\prime}+\cdots+k e_{i}^{\prime}
$$

for $i=1, \ldots, r-1$. Choose $v \in V \backslash V^{\prime}$. Since $V^{\prime}$ is a closed subset of $V$, one has

$$
\operatorname{dist}\left(v, V^{\prime}\right):=\inf \left\{\|v-x\|: x \in V^{\prime}\right\}>0 .
$$

There then exists $y \in V^{\prime}$ such that $\|v-y\| \leq(\sqrt{\alpha})^{-1} \operatorname{dist}\left(v, V^{\prime}\right)$. We set $e_{r}=v-y$. Clearly $\left(e_{1}, \ldots, e_{r-1}, e_{r}\right)$ forms a basis of $V$. It is sufficient to see that

$$
\left\|a_{1} e_{1}+\cdots+a_{r-1} e_{r-1}+e_{r}\right\| \geq \alpha \max \left\{\left|a_{1}\right|\left\|e_{1}\right\|, \ldots,\left|a_{r-1}\right|\left\|e_{r-1}\right\|,\left\|e_{r}\right\|\right\}
$$

for all $a_{1}, \ldots, a_{r-1} \in k$. Indeed, as $\left\|e_{r}\right\| \leq(\sqrt{\alpha})^{-1}\left\|a_{1} e_{1}+\cdots+a_{r-1} e_{r-1}+e_{r}\right\|$, we have

$$
\alpha\left\|e_{r}\right\| \leq \sqrt{\alpha}\left\|e_{r}\right\| \leq\left\|a_{1} e_{1}+\cdots+a_{r-1} e_{r-1}+e_{r}\right\| .
$$

If $\left\|a_{1} e_{1}+\cdots+a_{r-1} e_{r-1}\right\| \leq\left\|e_{r}\right\|$, then

$$
\begin{aligned}
\left\|a_{1} e_{1}+\cdots+a_{r-1} e_{r-1}+e_{r}\right\| & \geq \sqrt{\alpha}\left\|e_{r}\right\| \geq \sqrt{\alpha}\left\|a_{1} e_{1}+\cdots+a_{r-1} e_{r-1}\right\| \\
& \geq \sqrt{\alpha}\left(\sqrt{\alpha} \max \left\{\left|a_{1}\right|\left\|e_{1}\right\|, \ldots,\left|a_{r-1}\right|\left\|e_{r-1}\right\|\right\}\right) \\
& =\alpha \max \left\{\left|a_{1}\right|\left\|e_{1}\right\|, \ldots,\left|a_{r-1}\right|\left\|e_{r-1}\right\|\right\} .
\end{aligned}
$$

Otherwise,

$$
\begin{aligned}
\left\|a_{1} e_{1}+\cdots+a_{r-1} e_{r-1}+e_{r}\right\| & =\left\|a_{1} e_{1}+\cdots+a_{r-1} e_{r-1}\right\| \\
& \geq \sqrt{\alpha} \max \left\{\left|a_{1}\right|\left\|e_{1}\right\|, \ldots,\left|a_{r-1}\right|\left\|e_{r-1}\right\|\right\} \\
& \geq \alpha \max \left\{\left|a_{1}\right|\left\|e_{1}\right\|, \ldots,\left|a_{r-1}\right|\left\|e_{r-1}\right\|\right\},
\end{aligned}
$$

as required.

For the second assertion, it is sufficient to show (1) in Lemma[1.5]below because it implies that the set $\left\{\|v-x\| \mid x \in V^{\prime}\right\}$ has the minimal value.

Remark 1.4. We assume that $k$ is not complete. Let $\gamma \in \widehat{k} \backslash k$, we define a norm $\|\cdot\|_{\gamma}$ on $k^{2}$ by

$$
\forall(a, b) \in k^{2}, \quad\|(a, b)\|_{\gamma}:=|a+b \gamma| .
$$

Then there is no positive constant $C$ such that $\|(a, b)\|_{\gamma} \geq C \max \{|a|,|b|\}$ for all $a, b \in k$. In particular, for any $\alpha \in(0,1]$, there is no $\alpha$-orthogonal basis of $k^{2}$ with respect to $\|\cdot\|_{\gamma}$. Indeed, we assume the contrary. We can find a sequence $\left\{a_{n}\right\}$ in $k$ with $\lim _{n \rightarrow \infty}\left|a_{n}-\gamma\right|=0$. On the other hand,

$$
\left|a_{n}-\gamma\right|=\left\|\left(a_{n},-1\right)\right\|_{\gamma} \geq C \max \left\{\left|a_{n}\right|, 1\right\} \geq C
$$

for all $n$. This is a contradiction. Note that the norm $\|\cdot\|_{\gamma}$ extends by continuity to a map $\widehat{k}^{2} \rightarrow \mathbb{R}_{\geqslant 0}$ sending $(a, b) \in \widehat{k}^{2}$ to $|a+b \gamma|$. But this map is a semi-norm 
instead of a norm. Therefore, the hypothesis that the $\|\cdot\|$ extends to a norm on $V \otimes_{k} \widehat{k}$ is essential.

Lemma 1.5. (1) We assume that $|\cdot|$ is discrete. Then the set $\{\|v\| \mid v \in V \backslash\{0\}\}$ is discrete in $\mathbb{R}_{>0}$ (cf. [6. Proposition 2.5]).

(2) Let $M$ be a subspace of $V$ over $k$, and let $\|\cdot\|_{\hat{k}}$ be an ultrametric norm on $V \otimes_{k} \hat{k}$ such that $\|\cdot\|_{\hat{k}}$ is an extension of $\|\cdot\|$. Then, for $x \in V$ and $v^{\prime} \in M \otimes_{k} \hat{k}$, there exists $v \in M$ with $\left\|v^{\prime}+x\right\|_{\hat{k}}=\|v+x\|$. In particular, $\{\|v+x\| \mid v \in M\}=$ $\left\{\left\|v^{\prime}+x\right\|_{\hat{k}} \mid v^{\prime} \in M \otimes_{k} \hat{k}\right\}$ for any $x \in V$.

Proof. (1) Let us consider a map $\beta: V \backslash\{0\} \rightarrow \mathbb{R}_{>0} /\left|k^{\times}\right|$given by

$$
\beta(v)=\text { the class of }\|v\| \text { in } \mathbb{R}_{>0} /\left|k^{\times}\right| \text {. }
$$

For the assertion of (1), it is sufficient to see that $\beta(V \backslash\{0\})$ is finite. Let $\beta_{1}, \ldots, \beta_{l}$ be distinct elements of $\beta(V \backslash\{0\})$. We choose $v_{1}, \ldots, v_{l} \in V \backslash\{0\}$ with $\beta\left(v_{i}\right)=\beta_{i}$ for $i=1, \ldots, l$. If $i \neq j$, then $\left\|a_{i} v_{i}\right\| \neq\left\|a_{j} v_{j}\right\|$ for all $a_{i}, a_{j} \in k^{\times}$. Therefore, we obtain

$$
\left\|a_{1} v_{1}+\cdots+a_{l} v_{l}\right\|=\max \left\{\left\|a_{1} v_{1}\right\|, \ldots,\left\|a_{1} v_{l}\right\|\right\}
$$

for all $a_{1}, \ldots, a_{l} \in k$. In particular, $v_{1}, \ldots, v_{l}$ are linearly independent. Therefore, we have $\#(\beta(V \backslash\{0\})) \leq \operatorname{dim}_{k} V$.

(2) Clearly we may assume that $\left\|v^{\prime}+x\right\|_{\hat{k}} \neq 0$ because $\left(M \otimes_{k} \hat{k}\right) \cap V=M$. Since any $k$-linear isomorphism $k^{e} \rightarrow M$ is a homeomorphism (where $e$ is the rank of $M)$, we obtain that $M$ is dense in $M \otimes_{k} \hat{k}$. Therefore, there exists a sequence $\left(v_{n}\right)_{n \in \mathbb{N}}$ in $M$ which converges to $v^{\prime}$, so that $\lim _{n \rightarrow \infty}\left(v_{n}+x\right)=v^{\prime}+x$. Since $\|\cdot\|_{\hat{k}}$ is ultrametric and $\left\|v^{\prime}+x\right\|_{\hat{k}} \neq 0$, we obtain that $\left\|v_{n}+x\right\|=\left\|v^{\prime}+x\right\|_{\hat{k}}$ for sufficiently positive $n$, as required.

Proposition 1.6. We assume that $|\cdot|$ is discrete. Then

$$
(V,\|\cdot\|)_{\leq 1}:=\{v \in V \mid\|v\| \leq 1\}
$$

is a finitely generated $\mathfrak{o}_{k}$-module.

Proof. By Proposition 1.3, there is an orthogonal basis $\left(e_{1}, \ldots, e_{r}\right)$ of $V$ with respect to $\|\cdot\|$. We choose $\lambda_{i} \in k^{\times}$such that

$$
\left|\lambda_{i}\right|=\inf \left\{|\lambda| \mid \lambda \in k^{\times} \text {and }\left\|e_{i}\right\| \leq|\lambda|\right\} .
$$

We set $\omega_{i}=\lambda_{i}^{-1} e_{i}$. Note that $\omega_{i} \in(V,\|\cdot\|)_{\leq 1}$. We assume that $\|v\| \leq 1$ for $v=$ $a_{1} \omega_{1}+\cdots+a_{r} \omega_{r}\left(a_{1}, \ldots, a_{r} \in k\right)$. In order to see that $\left(\omega_{1}, \ldots, \omega_{r}\right)$ is a free basis of $(V,\|\cdot\|)_{\leq 1}$, it is sufficient to show that $\left|a_{i}\right| \leq 1$. Clearly we may assume that $a_{i} \neq 0$. Since $\left(\omega_{1}, \ldots, \omega_{r}\right)$ is an orthogonal basis, $\|x\| \leq 1$ implies $\left|a_{i}\right|\left\|\omega_{i}\right\| \leq 1$, that is, $\left\|e_{i}\right\| \leq\left|\lambda_{i} / a_{i}\right|$, and hence $\left|\lambda_{i}\right| \leq\left|\lambda_{i} / a_{i}\right|$. Therefore, $\left|a_{i}\right| \leq 1$.

Proposition 1.7. Assume that $|\cdot|$ is discrete. Let $V$ be a finite dimensional vector space over $k$ and $W$ be a quotient vector space of $V$. Denote by $\pi: V \rightarrow W$ be the projection map. We equip $V \otimes_{k} \widehat{k}$ with a norm $\|\cdot\|_{V}$ and $W \otimes_{k} \widehat{k}$ with the quotient norm $\|\cdot\|_{W}$. Then for any $y \in W$ there is $x \in V$ such that $\pi(x)=y$ and $\|y\|_{W}=\|x\|_{V}$.

Proof. We may assume that $y \neq 0$ (the case where $y=0$ is trivial). We set $M=\operatorname{Ker}(\pi)$. Note that $M \otimes_{k} \hat{k}=\operatorname{Ker}\left(\pi_{\hat{k}}\right)$. By Lemma 1.5, $\{\|v\| \mid v \in V \backslash\{0\}\}$ is 
discrete in $\mathbb{R}_{>0}$ and

$$
\begin{aligned}
\left\{\left\|x^{\prime}\right\|_{V} \mid x^{\prime} \in V \otimes_{k} \hat{k} \text { and } \pi_{\hat{k}}\left(x^{\prime}\right)=y\right\}=\left\{\|x\|_{V} \mid x \in V \text { and } \pi(x)\right. & =y\} \\
& \subseteq\{\|v\| \mid v \in V \backslash\{0\}\} .
\end{aligned}
$$

Thus the proposition follows.

1.3.2. Scalar extension of norms. Let $V^{\prime}$ be a vector space over $k$ and $\|\cdot\|^{\prime}$ a norm of $V^{\prime}$.

Lemma 1.8. For $\phi \in \operatorname{Hom}_{k}\left(V, V^{\prime}\right)$, the set $\left\{\frac{\|\phi(v)\|^{\prime}}{\|v\|} \mid v \in V \backslash\{0\}\right\}$ is bounded from above.

Proof. Fix $\alpha \in(0,1)$. Let $\left(e_{1}, \ldots, e_{r}\right)$ be an $\alpha$-orthogonal basis of $V$ (cf. Proposition 1.3). We set

$$
C_{1}=\max \left\{\left\|\phi\left(e_{1}\right)\right\|^{\prime}, \ldots,\left\|\phi\left(e_{r}\right)\right\|^{\prime}\right\} \quad \text { and } \quad C_{2}=\min \left\{\left\|e_{1}\right\|, \ldots,\left\|e_{r}\right\|\right\} .
$$

Then, for $v=a_{1} e_{1}+\cdots+a_{r} e_{r} \in V \backslash\{0\}$,

$$
\begin{aligned}
\frac{\|\phi(v)\|^{\prime}}{\|v\|} & \leq \frac{\max \left\{\left|a_{1}\right|\left\|\phi\left(e_{1}\right)\right\|^{\prime}, \ldots,\left|a_{r}\right|\left\|\phi\left(e_{r}\right)\right\|^{\prime}\right\}}{\alpha \max \left\{\left|a_{1}\right|\left\|e_{1}\right\|, \ldots,\left|a_{r}\right|\left\|e_{r}\right\|\right\}} \\
& \leq \frac{\max \left\{\left|a_{1}\right| C_{1}, \ldots,\left|a_{r}\right| C_{1}\right\}}{\alpha \max \left\{\left|a_{1}\right| C_{2}, \ldots,\left|a_{r}\right| C_{2}\right\}}=\frac{C_{1}}{\alpha C_{2}},
\end{aligned}
$$

as desired.

By the above lemma, we define $\|\phi\|_{\operatorname{Hom}_{k}\left(V, V^{\prime}\right)}$ to be

$$
\|\phi\|_{\operatorname{Hom}_{k}\left(V, V^{\prime}\right)}:=\sup \left\{\frac{\|\phi(v)\|^{\prime}}{\|v\|} \mid v \in V \backslash\{0\}\right\} .
$$

Note that $\|\cdot\|_{\operatorname{Hom}_{k}\left(V, V^{\prime}\right)}$ yields a norm on $\operatorname{Hom}_{k}\left(V, V^{\prime}\right)$. We denote $\|\cdot\|_{\operatorname{Hom}_{k}(V, k)}$ by $\|\cdot\|^{\vee}$ (i.e. the case where $V^{\prime}=k$ and $\|\cdot\|^{\prime}=|\cdot|$ ).

Lemma 1.9. Let $W$ be a subspace of $V$ and $\psi \in W^{\vee}:=\operatorname{Hom}_{k}(W, k)$. For any $\alpha \in$ $(0,1)$, there is $\varphi \in V^{\vee}:=\operatorname{Hom}_{k}(V, k)$ such that $\left.\varphi\right|_{W}=\psi$ and

$$
\|\psi\|^{\vee} \leq\|\varphi\|^{\vee} \leq \alpha^{-1}\|\psi\|^{\vee} \text {. }
$$

Proof. Let $\left(e_{1}, \ldots, e_{r}\right)$ be an $\alpha$-orthogonal basis of $V$ such that $W=k e_{1}+\cdots+k e_{l}$ (cf. Proposition 1.3). We define $\varphi \in V^{\vee}$ to be

$$
\varphi\left(a_{1} e_{1}+\cdots+a_{r} e_{r}\right):=\psi\left(a_{1} e_{1}+\cdots+a_{l} e_{l}\right)
$$

for $a_{1}, \ldots, a_{r} \in k$. Then $\left.\varphi\right|_{W}=\psi$. Moreover, note that

$$
\begin{aligned}
\alpha\left\|a_{1} e_{1}+\cdots+a_{l} e_{l}\right\| \leq & \alpha \max \left\{\left|a_{1}\right|\left\|e_{1}\right\|, \ldots,\left|a_{l}\right|\left\|e_{l}\right\|\right\} \\
& \leq \alpha \max \left\{\left|a_{1}\right|\left\|e_{1}\right\|, \ldots,\left|a_{r}\right|\left\|e_{r}\right\|\right\} \leq\left\|a_{1} e_{1}+\cdots+a_{r} e_{r}\right\|,
\end{aligned}
$$

so that

$$
\frac{\left|\varphi\left(a_{1} e_{1}+\cdots+a_{r} e_{r}\right)\right|}{\left\|a_{1} e_{1}+\cdots+a_{r} e_{r}\right\|} \leq \alpha^{-1} \frac{\left|\psi\left(a_{1} e_{1}+\cdots+a_{l} e_{l}\right)\right|}{\left\|a_{1} e_{1}+\cdots+a_{l} e_{l}\right\|} \leq \alpha^{-1}\|\psi\|^{\vee}
$$

for all $a_{1}, \ldots, a_{r} \in k$ with $\left(a_{1}, \ldots, a_{l}\right) \neq(0, \ldots, 0)$. Thus the assertion follows.

Corollary 1.10. The natural homomorphism $V \rightarrow\left(V^{\vee}\right)^{\vee}$ is an isometry. 
Proof. We denote the norm of $\left(V^{\vee}\right)^{\vee}$ by $\|\cdot\|^{\prime}$, that is,

$$
\|v\|^{\prime}=\sup \left\{\frac{|\phi(v)|}{\|\phi\|^{\vee}} \mid \phi \in V^{\vee} \backslash\{0\}\right\} .
$$

Note that $|\phi(v)| \leq\|v\|\|\phi\|^{\vee}$ for all $v \in V$ and $\phi \in V^{\vee}$. In particular, $\|v\|^{\prime} \leq\|v\|$. For $v \in V \backslash\{0\}$, we set $W:=k v$ and choose $\psi \in W^{\vee}$ with $\psi(v)=1$. Then $\|\psi\|^{\vee}=1 /\|v\|$. For any $\alpha \in(0,1)$, by Lemma 1.9, there is $\varphi \in V^{\vee}$ such that $\left.\varphi\right|_{W}=\psi$ and $\|\varphi\|^{\vee} \leq \alpha^{-1}\|\psi\|^{\vee}$. As $|\varphi(v)| /\|\varphi\|^{\vee} \leq\|v\|^{\prime}$, we have $\alpha\|v\| \leq\|v\|^{\prime}$. Thus we obtain $\|v\| \leq\|v\|^{\prime}$ by taking $\alpha \rightarrow 1$.

Definition 1.11. Let $k^{\prime}$ be an extension field of $k$, and let $|\cdot|^{\prime}$ be a complete absolute value of $k^{\prime}$ which is an extension of $|\cdot|$. We set $V_{k^{\prime}}:=V \otimes_{k} k^{\prime}$. Identifying $V_{k^{\prime}}$ with

$$
\operatorname{Hom}_{k}\left(\operatorname{Hom}_{k}(V, k), k^{\prime}\right) \text {, }
$$

we can give a norm $\|\cdot\|_{k^{\prime}}$ of $V_{k^{\prime}}$, that is,

$$
\left\|v^{\prime}\right\|_{k^{\prime}}=\sup \left\{\frac{\left|(\phi \otimes 1)\left(v^{\prime}\right)\right|^{\prime}}{\|\phi\|^{\vee}} \mid \phi \in V^{\vee}\right\} .
$$

The norm $\|\cdot\|_{k^{\prime}}$ is called the scalar extension of $\|\cdot\|$. Note that $\|v \otimes 1\|_{k^{\prime}}=\|v\|$ for $v \in V$. Indeed, by Corollary 1.10 .

$$
\|v \otimes 1\|_{k^{\prime}}=\sup \left\{\frac{|\phi(v)|}{\|\phi\|^{\vee}} \mid \phi \in V^{\vee}\right\}=\|v\| .
$$

By definition, if $\|\cdot\|_{1}$ and $\|\cdot\|_{2}$ are two norms on $V$ such that $\|\cdot\|_{1} \leqslant\|\cdot\|_{2}$, then one has $\|\cdot\|_{1}^{\vee} \geqslant\|\cdot\|_{2}$ and hence $\|\cdot\|_{1, k^{\prime}} \leqslant\|\cdot\|_{2, k^{\prime}}$.

Proposition 1.12. For $\alpha \in(0,1]$, let $\left(e_{1}, \ldots, e_{r}\right)$ be an $\alpha$-orthogonal basis of $V$ with respect to $\|\cdot\|$. Then $\left(e_{1} \otimes 1, \ldots, e_{r} \otimes 1\right)$ also yields an $\alpha$-orthogonal basis of $V_{k^{\prime}}$ with respect to $\|\cdot\|_{k^{\prime}}$. In particular, $\|\cdot\|_{k^{\prime}}$ is actually the largest ultrametric norm on $V_{k^{\prime}}$ extending $\|\cdot\|$.

Proof. Let $\left(e_{1}^{\vee}, \ldots, e_{r}^{\vee}\right)$ be the dual basis of $\left(e_{1}, \ldots, e_{r}\right)$. For $a_{1}, \ldots, a_{r} \in k$ with $a_{i} \neq 0$,

$$
\frac{\left|\left(e_{i}^{\vee}\right)\left(a_{1} e_{1}+\cdots+a_{r} e_{r}\right)\right|}{\left\|a_{1} e_{1}+\cdots+a_{r} e_{r}\right\|} \leq \frac{\left|a_{i}\right|}{\alpha \max \left\{\left|a_{1}\right|\left\|e_{1}\right\|, \ldots,\left|a_{r}\right|\left\|e_{r}\right\|\right\}} \leq \frac{\left|a_{i}\right|}{\alpha\left|a_{i}\right|\left\|e_{i}\right\|}=\frac{1}{\alpha\left\|e_{i}\right\|},
$$

and hence $\left\|e_{i}^{\vee}\right\|^{\vee} \leq\left(\alpha\left\|e_{i}\right\|\right)^{-1}$. Therefore, for $a_{1}^{\prime}, \ldots, a_{r}^{\prime} \in k^{\prime}$,

$$
\begin{aligned}
\left\|a_{1}^{\prime} e_{1}+\cdots+a_{r}^{\prime} e_{r}\right\|_{k^{\prime}} & \geq \frac{\left|\left(e_{i}^{\vee} \otimes 1\right)\left(a_{1}^{\prime} e_{1}+\cdots+a_{r}^{\prime} e_{r}\right)\right|^{\prime}}{\left\|e_{i}^{\vee}\right\|^{\vee}} \\
& =\frac{\left|a_{i}^{\prime}\right|^{\prime}}{\left\|e_{i}^{\vee}\right\|^{\vee}} \geq \frac{\left|a_{i}^{\prime}\right|^{\prime}}{\left(\alpha\left\|e_{i}\right\|\right)^{-1}}=\alpha\left|a_{i}^{\prime}\right|^{\prime}\left\|e_{i}\right\| .
\end{aligned}
$$

Thus we have the first assertion.

Assume that $\|\cdot\|^{\prime}$ is another ultrametric norm on $V_{k^{\prime}}$ extending $\|\cdot\|$. If $\left(e_{1}, \ldots, e_{r}\right)$ is an $\alpha$-orthogonal basis of $V$, where $\alpha \in(0,1)$, by the first assertion of the proposition, we have

$$
\begin{aligned}
& \forall\left(a_{1}^{\prime}, \ldots, a_{r}^{\prime}\right) \in k^{\prime}, \quad \alpha\left\|a_{1}^{\prime} e_{1}+\cdots+a_{r}^{\prime} e_{r}\right\|^{\prime} \leqslant \alpha \max _{i \in\{1, \ldots, r\}}\left(\left|a_{i}\right|^{\prime}\left\|e_{i}\right\|^{\prime}\right) \\
& =\alpha \max _{i \in\{1, \ldots, r\}}\left(\left|a_{i}\right|^{\prime}\left\|e_{i}\right\|\right) \leqslant\left\|a_{1}^{\prime} e_{1}+\cdots+a_{r}^{\prime} e_{r}\right\|_{k^{\prime}} .
\end{aligned}
$$

Since $\alpha$ is arbitrary, we obtain $\|\cdot\|^{\prime} \leqslant\|\cdot\|_{k^{\prime}}$. 
Lemma 1.13. Let $k^{\prime \prime}$ be an extension field of $k^{\prime}$, and let $|\cdot|{ }^{\prime \prime}$ be a complete absolute value of $k^{\prime \prime}$ as an extension of $|\cdot|^{\prime}$. We set $V_{k^{\prime \prime}}:=V \otimes_{k} k^{\prime \prime}$. Note that $V_{k^{\prime \prime}}=V_{k^{\prime}} \otimes_{k^{\prime}} k^{\prime \prime}$. Let $\|\cdot\|_{k^{\prime \prime}}\left(\right.$ resp. $\left.\|\cdot\|_{k^{\prime}, k^{\prime \prime}}\right)$ be a norm of $V_{k^{\prime \prime}}$ obtained by the scalar extension of $\|\cdot\|$ on $V$ (resp. the scalar extension of $\|\cdot\|_{k^{\prime}}$ on $V_{k^{\prime}}$. Then $\|\cdot\|_{k^{\prime \prime}}=\|\cdot\|_{k^{\prime}, k^{\prime \prime}}$.

Proof. Since $\|\cdot\|_{k^{\prime}, k^{\prime \prime}}$ is an ultrametric norm on $V_{k^{\prime \prime}}$ extending $\|\cdot\|$, by Proposition 1.12 we have $\|\cdot\|_{k^{\prime}, k^{\prime \prime}} \leqslant\|\cdot\|_{k^{\prime \prime}}$. Moreover, since the restriction of $\|\cdot\|_{k^{\prime \prime}}$ on $V_{k^{\prime}}$ (which we denote by $\|\cdot\|^{\prime}$ ) extends the norm $\|\cdot\|$ on $V$, by the same reason we have $\|\cdot\|^{\prime} \leqslant\|\cdot\|_{k^{\prime}}$ and hence $\|\cdot\|^{\prime}=\|\cdot\|_{k^{\prime}}$. Therefore, still by Proposition 1.12 we have $\|\cdot\|_{k^{\prime \prime}} \leqslant\|\cdot\|_{k^{\prime}, k^{\prime \prime}}$ and hence $\|\cdot\|_{k^{\prime \prime}}=\|\cdot\|_{k^{\prime}, k^{\prime \prime}}$.

Lemma 1.14. Let $f: V \rightarrow W$ be a surjective homomorphism of finite-dimensional vector spaces over $k$. Let $\|\cdot\|_{V}$ and $\|\cdot\|_{W}$ be norms of $V$ and $W$, respectively. We assume that $\operatorname{dim}_{k} W=1$ and $\|\cdot\|_{W}$ is the quotient norm of $\|\cdot\|_{V}$ induced by the surjective map $f: V \rightarrow W$. We set $V_{k^{\prime}}:=V \otimes_{k} k^{\prime}$ and $W_{k^{\prime}}:=W \otimes_{k} k^{\prime}$. Let $\|\cdot\|_{V, k^{\prime}}$ and $\|\cdot\|_{W, k^{\prime}}$ be the norms of $V_{k^{\prime}}$ and $W_{k^{\prime}}$ obtained by the scalar extensions of $\left\|_{V} \cdot\right\|_{V}$ and $\|\cdot\|_{W}$, respectively. Then $\|\cdot\|_{W, k^{\prime}}$ is the quotient norm of $\|\cdot\|_{V, k^{\prime}}$ in terms of the surjection $f_{k^{\prime}}:=f \otimes \operatorname{id}_{k^{\prime}}: V_{k^{\prime}} \rightarrow W_{k^{\prime}}$.

Proof. Let $\|\cdot\|_{W_{k^{\prime}}}^{\prime}$ be the quotient norm of $\|\cdot\|_{V, k^{\prime}}$ with respect to the surjection $f_{k^{\prime}}: V_{k^{\prime}} \rightarrow W_{k^{\prime}}$. Let $e$ be an non-zero element of $W$. As $\|e\|_{W, k^{\prime}}=\|e\|_{W}$, it is sufficient to show that $\|e\|_{W_{k^{\prime}}}^{\prime}=\|e\|_{W}$. Note that

$$
\{v \in V \mid f(v)=e\} \subseteq\left\{v^{\prime} \in V_{k^{\prime}} \mid f_{k^{\prime}}\left(v^{\prime}\right)=e\right\},
$$

so that we have $\|e\|_{W} \geq\|e\|_{W_{k^{\prime}}}^{\prime}$. In the following, we prove the inequality $\|e\|_{W} \leq\|e\|_{W_{k^{\prime}}}^{\prime}$. For $\epsilon>0$, let $\left(e_{1}, \ldots, e_{r}\right)$ be an $e^{-\epsilon}$-orthogonal basis of $V$ such that $\left(e_{2}, \ldots, e_{r}\right)$ forms a basis of $\operatorname{Ker}(f)$. Clearly we may assume that $f\left(e_{1}\right)=e$. Then

$$
\begin{aligned}
\|e\|_{W_{k^{\prime}}}^{\prime} & =\inf \left\{\left\|e_{1}+a_{2}^{\prime} e_{2}+\cdots+a_{r}^{\prime} e_{r}\right\|_{V, k^{\prime}} \mid a_{2}^{\prime}, \ldots, a_{r}^{\prime} \in k^{\prime}\right\} \\
& \geq \inf \left\{e^{-\epsilon} \max \left\{\left\|e_{1}\right\|,\left|a_{2}^{\prime}\right|^{\prime}\left\|e_{2}\right\|_{V}, \ldots,\left|a_{r}^{\prime}\right|^{\prime}\left\|e_{r}\right\|_{V}\right\} \mid a_{2}^{\prime}, \ldots, a_{r}^{\prime} \in k^{\prime}\right\} \\
& \geq e^{-\epsilon}\left\|e_{1}\right\| \geq e^{-\epsilon}\|e\|_{W} .
\end{aligned}
$$

Therefore, we have $\|e\|_{W_{k^{\prime}}}^{\prime} \geq\|e\|_{W}$ by taking $\epsilon \rightarrow 0$.

Lemma 1.15. We assume that the absolute value $|\cdot|$ of $k$ is trivial. Let $(V,\|\cdot\|)$ be a finite-dimensional normed vector space over $(k,|\cdot|)$. Then we have the following:

(1) The set $\{\|v\| \mid v \in V\}$ is a finite set.

(2) Let $k^{\prime}$ be a field and $|\cdot|^{\prime}$ a complete and non-trivial absolute value of $k^{\prime}$ such that $k \subseteq k^{\prime}$ and $|\cdot|^{\prime}$ is an extension of $|\cdot|$. Let $\mathfrak{o}_{k^{\prime}}$ be the valuation ring of $\left(k^{\prime},|\cdot|^{\prime}\right)$ and $\mathfrak{m}_{k^{\prime}}$ the maximal ideal of $\mathfrak{o}_{k^{\prime}}$. We assume the following:

(i) The natural map $k \rightarrow \mathfrak{o}_{k^{\prime}}$ induces an isomorphism $k \stackrel{\sim}{\longrightarrow} \mathfrak{o}_{k^{\prime}} / \mathfrak{m}_{k^{\prime}}$.

(ii) If an equation $\left|a^{\prime}\right|^{\prime}=\|v\| /\left\|v^{\prime}\right\|$ holds for some $a^{\prime} \in k^{\prime \times}$ and $v, v^{\prime} \in$ $V \backslash\{0\}$, then $\|v\|=\left\|v^{\prime}\right\|$.

Let $\|\cdot\|^{\prime}$ be a norm of $V_{k^{\prime}}:=V \otimes_{k} k^{\prime}$ over $\left(k^{\prime},|\cdot|^{\prime}\right)$ such that $\|v\|=\|v \otimes 1\|^{\prime}$ for all $v \in V$. If $\left(e_{1}, \ldots, e_{r}\right)$ is an orthogonal basis of $(V,\|\cdot\|)$, then $\left(e_{1}, \ldots, e_{r}\right)$ forms an orthogonal basis of $\left(V_{k^{\prime}},\|\cdot\|^{\prime}\right)$. In particular, $\|\cdot\|^{\prime}=\|\cdot\|_{k^{\prime}}$. 
Proof. (1) Let $\left(e_{1}, \ldots, e_{r}\right)$ be an orthogonal basis of $(V,\|\cdot\|)$ (cf. Proposition 1.3).

Then

$$
\left\|a_{1} e_{1}+\cdots+a_{r} e_{r}\right\|=\max \left\{\left|a_{1}\right|\left\|e_{1}\right\|, \ldots,\left|a_{r}\right|\left\|e_{r}\right\|\right\}
$$

for all $a_{1}, \ldots, a_{r} \in k$, so that

$$
\left\|a_{1} e_{1}+\cdots+a_{r} e_{r}\right\| \in\left\{0,\left\|e_{1}\right\|, \ldots,\left\|e_{r}\right\|\right\} .
$$

(2) First we assume that

$$
\left\|e_{1}\right\|=\cdots=\left\|e_{r}\right\|=c .
$$

Then, for any $v \in V$,

$$
\|v\|= \begin{cases}c & \text { if } v \neq 0 \\ 0 & \text { if } v=0\end{cases}
$$

Let us see that

$$
\left\|a_{1}^{\prime} e_{1}+\cdots+a_{r}^{\prime} e_{r}\right\|^{\prime}=c \max \left\{\left|a_{1}^{\prime}\right|^{\prime}, \ldots,\left|a_{r}^{\prime}\right|^{\prime}\right\}
$$

for $a_{1}^{\prime}, \ldots, a_{r}^{\prime} \in k^{\prime}$. Clearly we may assume that

$$
\left(a_{1}^{\prime}, \ldots, a_{r}^{\prime}\right) \neq(0, \ldots, 0) .
$$

We set $\gamma:=\max \left\{\left|a_{1}^{\prime}\right|^{\prime}, \ldots,\left|a_{r}^{\prime}\right|^{\prime}\right\}$. We fix $\omega \in k^{\prime}$ with $|\omega|^{\prime}=\gamma$. By the assumption (i), for each $j=1, \ldots, r$, we can find $a_{j} \in k$ and $b_{j}^{\prime} \in k^{\prime}$ such that

$$
a_{j}^{\prime}=a_{j} \omega+b_{j}^{\prime} \text { and }\left|b_{j}^{\prime}\right|^{\prime}<\gamma .
$$

Note that

$$
a_{1}^{\prime} e_{1}+\cdots+a_{r}^{\prime} e_{r}=\omega\left(\sum_{j=1}^{r} a_{j} e_{j}\right)+b_{1}^{\prime} e_{1}+\cdots+b_{r}^{\prime} e_{r} .
$$

Moreover, as $\sum_{j=1}^{r} a_{j} e_{j} \neq 0$, we have

$$
\left\|\omega\left(\sum_{j=1}^{r} a_{j} e_{j}\right)\right\|^{\prime}=\gamma\left\|\sum_{j=1}^{r} a_{j} e_{j}\right\|=c \gamma
$$

and

$$
\left\|b_{1}^{\prime} e_{1}+\cdots+b_{r}^{\prime} e_{r}\right\|^{\prime} \leq c \max \left\{\left|b_{1}^{\prime}\right|^{\prime}, \ldots,\left|b_{r}^{\prime}\right|^{\prime}\right\}<c \gamma
$$

Therefore,

$$
\left\|a_{1}^{\prime} e_{1}+\cdots+a_{r}^{\prime} e_{r}\right\|^{\prime}=c \gamma=c \max \left\{\left|a_{1}^{\prime}\right|^{\prime}, \ldots,\left|a_{r}^{\prime}\right|^{\prime}\right\} .
$$

In general, we take positive numbers $c_{1}<\cdots<c_{b}$ and non-empty subsets $I_{1}, \ldots, I_{b}$ of $\{1, \ldots, r\}$ such that $\left\{\left\|e_{l}\right\| \mid l \in I_{s}\right\}=\left\{c_{s}\right\}$ for $s=1, \ldots, b$ and $I_{1} \cup$ $\cdots \cup I_{b}=\{1, \ldots, r\}$. Note that $I_{s} \cap I_{s^{\prime}}=\varnothing$ for $s \neq s^{\prime}$. Let us consider

$$
x=a_{1}^{\prime} e_{1}+\cdots+a_{r}^{\prime} e_{r}=\sum_{s=1}^{b} x_{s} \in V_{k^{\prime}} \quad\left(a_{1}^{\prime}, \ldots, a_{r}^{\prime} \in k^{\prime}\right),
$$

where $x_{s}=\sum_{l \in I_{s}} a_{l}^{\prime} e_{l}$. Note that $\left(e_{l}\right)_{l \in I_{s}}$ forms an orthogonal basis of $\bigoplus_{l \in I_{s}} k e_{l}$ and $\left\|e_{l}\right\|=c_{s}$ for all $l \in I_{s}$. Therefore, by the above observation,

$$
\left\|x_{s}\right\|^{\prime}=c_{s} \max _{l \in I_{s}}\left\{\left|a_{l}^{\prime}\right|^{\prime}\right\}=\max _{l \in I_{s}}\left\{\left\|a_{l}^{\prime} e_{l}\right\|^{\prime}\right\}
$$

so that it is sufficient to see that

$$
\|x\|^{\prime}=\max _{s=1, \ldots, b}\left\{\left\|x_{s}\right\|^{\prime}\right\} .
$$


Clearly we may assume that $x \neq 0$. We set

$$
\Sigma:=\left\{s \in\{1, \ldots, b\} \mid x_{s} \neq 0\right\} .
$$

For $s, s^{\prime} \in \Sigma$ with $s \neq s^{\prime}$, we have $\left\|x_{s}\right\|^{\prime} \neq\left\|x_{s^{\prime}}\right\|^{\prime}$. Indeed, we choose $l_{s} \in I_{s}$ and $l_{s^{\prime}} \in I_{s^{\prime}}$ with $\left\|x_{s}\right\|^{\prime}=\left\|a_{l_{s}}^{\prime} e_{l_{s}}\right\|^{\prime}$ and $\left\|x_{s^{\prime}}\right\|^{\prime}=\left\|a_{l_{s^{\prime}}}^{\prime} e_{l_{s^{\prime}}}\right\|^{\prime}$. If $\left\|x_{s}\right\|^{\prime}=\left\|x_{s^{\prime}}\right\|^{\prime}$, then

$$
\left|a_{l_{s}}^{\prime} / a_{l_{s^{\prime}}}^{\prime}\right|^{\prime}=\left\|e_{l_{s^{\prime}}}\right\| /\left\|e_{l_{s}}\right\|
$$

so that, by the assumption (ii), $\left\|e_{l_{s^{\prime}}}\right\|=\left\|e_{l_{s}}\right\|$, which is a contradiction. Therefore,

$$
\|x\|^{\prime}=\left\|\sum_{s \in \Sigma} x_{s}\right\|^{\prime}=\max _{s \in \Sigma}\left\{\left\|x_{s}\right\|^{\prime}\right\}=\max _{s=1, \ldots, b}\left\{\left\|x_{s}\right\|^{\prime}\right\},
$$

as required.

Remark 1.16. We assume that $|\cdot|^{\prime}$ is discrete and

$$
\left|a^{\prime}\right|^{\prime}=\exp \left(-\alpha \operatorname{ord}_{\mathfrak{o}_{k^{\prime}}}\left(a^{\prime}\right)\right) \quad\left(a^{\prime} \in k^{\prime}\right)
$$

for $\alpha \in \mathbb{R}_{>0}$. If

$$
\alpha \notin \bigcup_{v, v^{\prime} \in V \backslash\{0\}} \mathbb{Q}\left(\log \|v\|-\log \left\|v^{\prime}\right\|\right),
$$

then the assumption (ii) holds. Indeed, we suppose that $\left|a^{\prime}\right|^{\prime}=\|v\| /\left\|v^{\prime}\right\|$ for some $a^{\prime} \in k^{\prime \times}$ and $v, v^{\prime} \in V \backslash\{0\}$. Then

$$
-\alpha \operatorname{ord}_{\mathfrak{o}_{k^{\prime}}}\left(a^{\prime}\right)=\log \|v\|-\log \left\|v^{\prime}\right\|,
$$

so that $\operatorname{ord}_{\mathfrak{o}_{k^{\prime}}}\left(a^{\prime}\right)=0$, and hence $\|v\|=\left\|v^{\prime}\right\|$, as required.

1.3.3. Lattices and norms. From now on and until the end of the subsection, we assume that $|\cdot|$ is non-trivial. Let $\mathscr{V}$ be an $\mathfrak{o}_{k}$-submodule of $V$. We say that $\mathscr{V}$ is a lattice of $V$ if $\mathscr{V} \otimes_{\mathfrak{o}_{k}} k=V$ and

$$
\sup \left\{\|v\|_{0} \mid v \in \mathscr{V}\right\}<\infty
$$

for some norm $\|\cdot\|_{0}$ of $V$. Note that the condition $\sup \left\{\|v\|_{0} \mid v \in \mathscr{V}\right\}<\infty$ does not depend on the choice of the norm $\|\cdot\|_{0}$ since all norms on $V$ are equivalent. For a lattice $\mathscr{V}$ of $V$, we define $\|\cdot\|_{\mathscr{V}}$ to be

$$
\|v\|_{\mathscr{V}}:=\inf \left\{|a|^{-1} \mid a \in k^{\times} \text {and } a v \in \mathscr{V}\right\} .
$$

Note that $\|\cdot\|_{\mathscr{V}}$ forms a norm of $V$. Moreover, for a norm $\|\cdot\|$ of $V$,

$$
(V,\|\cdot\|)_{\leq 1}:=\{v \in V \mid\|v\| \leq 1\}
$$

is a lattice of $V$.

Proposition 1.17. Let $\mathscr{V}$ be a lattice of $V$. We assume that, as an $\mathfrak{o}_{k}$-module, $\mathscr{V}$ admits a free basis $\left(e_{1}, \ldots, e_{r}\right)$. Then $\left(e_{1}, \ldots, e_{r}\right)$ is an orthonormal basis of $V$ with respect to $\|\cdot\|_{\mathscr{V}}$.

Proof. For $v=a_{1} e_{1}+\cdots+a_{r} e_{r} \in V$ and $a \in k^{\times}$,

$$
\begin{aligned}
a v \in \mathscr{V} & \Longleftrightarrow a a_{i} \in \mathfrak{o}_{k} \text { for all } i=1, \ldots, r \\
& \Longleftrightarrow\left|a_{i}\right| \leq|a|^{-1} \text { for all } i=1, \ldots, r \\
& \Longleftrightarrow \max \left\{\left|a_{1}\right|, \ldots,\left|a_{r}\right|\right\} \leq|a|^{-1},
\end{aligned}
$$

so that $\|v\|_{\mathscr{V}}=\max \left\{\left|a_{1}\right|, \ldots,\left|a_{r}\right|\right\}$. 
Let us consider the following lemmas.

Lemma 1.18. A subgroup $G$ of $(\mathbb{R},+)$ is either discrete or dense in $\mathbb{R}$.

Proof. Clearly we may assume that $G \neq\{0\}$, so that $G \cap \mathbb{R}_{>0} \neq \varnothing$. We set $\delta=\inf \left(G \cap \mathbb{R}_{>0}\right)$. If $\delta \in G \cap \mathbb{R}_{>0}$, then $G=\mathbb{Z} \delta$. Indeed, for $g \in G$, let $n$ be an integer such that $n \leq g / \delta<n+1$. Thus $0 \leq g-n \delta<\delta$, and hence $g=n \delta$. Therefore, $G$ is discrete.

Next we assume that $\delta \notin G \cap \mathbb{R}_{>0}$. Then there is a sequence $\left\{\delta_{n}\right\}_{n=1}^{\infty}$ in $G \cap \mathbb{R}_{>0}$ such that $\delta_{n}>\delta_{n+1}$ for all $n$ and $\lim _{n \rightarrow \infty} \delta_{n}=\delta$. If we set $a_{n}=\delta_{n}-\delta_{n+1}$, then $a_{n} \in G \cap \mathbb{R}_{>0}$ and $\lim _{n \rightarrow \infty} a_{n}=0$. For an open interval $(\alpha, \beta)$ of $\mathbb{R}(\alpha<\beta)$, we choose $a_{n}$ and an integer $m$ such that $a_{n}<\beta-\alpha$ and $m<\beta / a_{n} \leq m+1$. Then we have $m a_{n}<\beta$ and

$$
\alpha<\beta-a_{n} \leq(m+1) a_{n}-a_{n}=m a_{n},
$$

so that $m a_{n} \in(\alpha, \beta) \cap G$. Thus $G$ is dense.

Lemma 1.19. Let $\|\cdot\|$ be a norm of $V$ and $\mathscr{V}:=(V,\|\cdot\|)_{\leq 1}$. Then

$$
\|v\|_{\mathscr{V}}=\inf \left\{|b| \mid b \in k^{\times} \text {and }\|v\| \leq|b|\right\} .
$$

Moreover, $\|\cdot\| \leq\|\cdot\|_{\mathscr{V}}$ and $\|v\|_{\mathscr{V}}<|\alpha|\|v\|$ for all $\alpha \in k^{\times}$with $|\alpha|>1$ and $v \in V \backslash\{0\}$.

Proof. The first assertion is obvious because, for $a \in k^{\times}, a v \in \mathscr{V}$ if and only if $\|v\| \leq|a|^{-1}$.

For $v \in V$, let $a \in k^{\times}$with $a v \in \mathscr{V}$. Then $\|a v\| \leq 1$, that is, $\|v\| \leq|a|^{-1}$, and hence $\|v\| \leq\|v\|_{\mathscr{V}}$.

Finally we consider the second inequality, that is, $\|v\|_{\mathscr{V}}<|\alpha|\|v\|$ for $v \in$ $V \backslash\{0\}$. As $|\alpha|^{-1}<1$, there is $\epsilon>0$ with $|\alpha|^{-1} e^{\epsilon}<1$. By the first assertion, we can choose $b \in k^{\times}$such that $\|v\| \leq|b| \leq e^{\epsilon}\|v\|_{\mathscr{V}}$. If $\|v\| \leqslant\left|b \alpha^{-1}\right|$, then

$$
\|v\|_{\mathscr{V}} \leq|b||\alpha|^{-1} \leq e^{\epsilon}\|v\|_{\mathscr{V}}|\alpha|^{-1} \text {. }
$$

Thus $1 \leq e^{\epsilon}|\alpha|^{-1}$. This is a contradiction, so that $\|v\|>\left|b \alpha^{-1}\right|$. Therefore,

$$
\|v\|_{\mathscr{V}} \leq|b|<|\alpha|\|v\|
$$

as required.

Proposition 1.20. We assume that $|\cdot|$ is discrete. Then we have the following:

(1) Every lattice $\mathscr{V}$ of $V$ is a finitely generated $\mathfrak{o}_{k}$-module.

(2) If we set $\mathscr{V}:=(V,\|\cdot\|)_{\leq 1}$ for a norm of $\|\cdot\|$ of $V$, then $\|v\| \leq\|v\|_{\mathscr{V}}<|\omega|^{-1}\|v\|$ for $v \in V \backslash\{0\}$.

Proof. (1) By Proposition $1.6\left(V,\|\cdot\|_{\mathscr{V}}\right)_{\leq 1}$ is a finitely generated $\mathfrak{o}_{k}$-module. Moreover, note that $\mathscr{V} \subseteq\left(V,\|\cdot\|_{\mathscr{V}}\right)_{\leq 1}$. Thus we have (1) because $\mathfrak{o}_{k}$ is noetherian.

(2) follows from Lemma 1.19

Proposition 1.21. We assume that $|\cdot|$ is not discrete. If we set $\mathscr{V}:=(V,\|\cdot\|)_{\leq 1}$ for a norm of $\|\cdot\|$ of $V$, then $\|\cdot\|=\|\cdot\|_{\mathscr{V}}$.

Proof. By Lemma 1.18, we can find a sequence $\left\{\beta_{n}\right\}_{n=1}^{\infty}$ such that $\left|\beta_{n}\right|>1$ and $\lim _{n \rightarrow \infty}\left|\beta_{n}\right|=1$. On the other hand, by Lemma 1.19 .

$$
\|\cdot\| \leq\|\cdot\|_{\mathscr{V}} \leq\left|\beta_{n}\right|\|\cdot\| \text {. }
$$

Therefore the assertion follows. 
Proposition 1.22. We assume that the absolute value $|\cdot|$ is not discrete. Let $\|\cdot\|$ be a norm of $V$ and $\mathscr{V}:=(V,\|\cdot\|)_{\leq 1}$. For any $\epsilon>0$, there is a sub-lattice $\mathscr{V}^{\prime}$ of $\mathscr{V}$ such that $\mathscr{V}^{\prime}$ is finitely generated over $\mathfrak{o}_{k}$ and $\|\cdot\| \leq\|\cdot\|_{\mathscr{V}^{\prime}} \leq e^{\epsilon}\|\cdot\|$.

Proof. Let $\left(e_{1}, \ldots, e_{r}\right)$ be an $e^{-\epsilon / 2}$-orthogonal basis of $V$ with respect to $\|\cdot\|$ (cf. Proposition [1.3). As $\|\cdot\|=\|\cdot\|_{\mathscr{V}}$ by Proposition 1.21, we can find $\lambda_{i} \in k^{\times}$such that $\left\|e_{i}\right\| \leq\left|\lambda_{i}\right| \leq e^{\epsilon / 2}\left\|e_{i}\right\|$ for each $i$. We set $\omega_{i}:=\lambda_{i}^{-1} e_{i}(i=1, \ldots, r)$ and $\mathscr{V}^{\prime}:=\mathfrak{o}_{k} \omega_{1}+\cdots+\mathfrak{o}_{k} \omega_{r}$. Note that $\omega_{i} \in \mathscr{V}$ for all $i$, that is, $\mathscr{V}^{\prime}$ is a sub-lattice of $\mathscr{V}$ and $\mathscr{V}^{\prime}$ is finitely generated over $\mathfrak{o}_{k}$. For $c_{1}, \ldots, c_{r} \in k$, by Proposition 1.17

$$
\begin{aligned}
\left\|c_{1} e_{1}+\cdots+c_{r} e_{r}\right\|_{\mathscr{V}^{\prime}} & =\left\|c_{1} \lambda_{1} \omega_{1}+\cdots+c_{r} \lambda_{r} \omega_{r}\right\|_{\mathscr{V}^{\prime}}=\max \left\{\left|c_{1} \lambda_{1}\right|, \ldots,\left|c_{r} \lambda_{r}\right|\right\} \\
& \leq e^{\epsilon / 2}\left\{\left|c_{1}\right|\left\|e_{1}\right\|, \ldots,\left|c_{r}\right|\left\|e_{r}\right\|\right\} \leq e^{\epsilon}\left\|c_{1} e_{1}+\cdots+c_{r} e_{r}\right\|,
\end{aligned}
$$

so that we have $\|\cdot\|_{\mathscr{V}^{\prime}} \leq e^{\epsilon}\|\cdot\|$.

\section{SEMINORM AND INTEGRAL EXTENSION}

Let $\mathscr{A}$ be a finitely generated $\mathfrak{o}_{k}$-algebra, which contains $\mathfrak{o}_{k}$ as a subring. We set $A:=\mathscr{A} \otimes_{\mathfrak{o}_{k}} k$. Note that $A$ coincides with the localization of $\mathscr{A}$ with respect to $S:=\mathfrak{o}_{k} \backslash\{0\}$. Let $\operatorname{Spec}(A)^{\text {an }}$ be the analytification of $\operatorname{Spec}(A)$, that is, the set of all seminorms of $A$ over the absolute value of $k$. For $x \in \operatorname{Spec}(A)^{\text {an }}$, let $\mathfrak{o}_{x}$ and $\mathfrak{m}_{x}$ be the valuation ring of $\left(\hat{\kappa}(x),|\cdot|_{x}\right)$ and the maximal ideal of $\mathfrak{o}_{x}$, respectively (see $\$ 1.1 .3$ for the definition of $\hat{\kappa}(x)$ ). We denote the natural homomorphism $A \rightarrow \hat{\kappa}(x)$ by $\varphi_{x}$. It is easy to see that the following are equivalent:

(1) $\operatorname{Spec}(\hat{\kappa}(x)) \rightarrow \operatorname{Spec}(A)$ extends to $\operatorname{Spec}\left(\mathfrak{o}_{x}\right) \rightarrow \operatorname{Spec}(\mathscr{A})$, that is, there is a ring homomorphism $\tilde{\varphi}_{x}: \mathscr{A} \rightarrow \mathfrak{o}_{x}$ such that the following diagram is commutative:

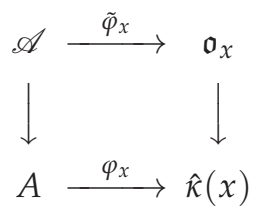

(2) $|a|_{x} \leq 1$ for all $a \in \mathscr{A}$.

Moreover, under the above conditions, the image of $\mathfrak{m}_{x}$ of $\operatorname{Spec}\left(\mathfrak{o}_{x}\right)$ is given by $\tilde{\varphi}_{x}^{-1}\left(\mathfrak{m}_{x}\right)=\left(\mathscr{A},|\cdot|_{x}\right)_{<1}$, and $\left(\mathscr{A},|\cdot|_{x}\right)_{<1} \in \operatorname{Spec}(\mathscr{A})_{0}$, where

$$
\left\{\begin{array}{l}
\left(\mathscr{A},|\cdot|_{x}\right)_{<1}:=\left\{\left.a \in \mathscr{A}|| a\right|_{x}<1\right\}, \\
\operatorname{Spec}(\mathscr{A})_{0}:=\left\{P \in \operatorname{Spec}(\mathscr{A}) \mid P \cap \mathfrak{o}_{k}=\mathfrak{m}_{k}\right\} .
\end{array}\right.
$$

Let $\operatorname{Spec}(A)_{\mathscr{A}}^{\text {an }}$ be the set of all $x \in \operatorname{Spec}(A)^{\text {an }}$ such that the above condition (2) is satisfied. The map $r_{\mathscr{A}}: \operatorname{Spec}(A)_{\mathscr{A}}^{\mathrm{an}} \rightarrow \operatorname{Spec}(\mathscr{A})$ 。 given by

$$
x \mapsto\left(\mathscr{A},|\cdot|_{x}\right)_{<1}
$$

is called the reduction map (cf. \$1.1.6). Note that the reduction map is surjective (cf. [2. Proposition 2.4.4] or [10, 4.13 and Proposition 4.14]).

Theorem 2.1. If we set $\mathscr{B}:=\{\alpha \in A \mid \alpha$ is integral over $\mathscr{A}\}$, then

$$
\mathscr{B}=\bigcap_{x \in \operatorname{Spec}(A)_{\mathscr{A}}^{\mathrm{an}}}(A,|\cdot| x)_{\leq 1}
$$

where $\left(A,|\cdot|_{x}\right)_{\leq 1}:=\left\{\left.\alpha \in A|| \alpha\right|_{x} \leq 1\right\}$. 
Proof. First let us see that $\mathscr{B} \subseteq(A,|\cdot|)_{\leq 1}$ for all $x \in \operatorname{Spec}(A)_{\mathscr{A}}^{\text {an }}$. If $a \in \mathscr{B}$, then there are $a_{1}, \ldots, a_{n} \in \mathscr{A}$ such that $a^{n}+a_{1} a^{n-1}+\cdots+a_{n}=0$. We assume that $|a|_{x}>1$. Then

$$
\begin{aligned}
|a|_{x}^{n} & =\left|a^{n}\right|_{x}=\left|a_{1} a^{n-1}+\cdots+a_{n}\right|_{x} \leq \max _{i=1, \ldots, n}\left\{\left|a_{i}\right|_{x}|a|_{x}^{n-i}\right\} \\
& \leq \max _{i=1, \ldots, n}\left\{|a|_{x}^{n-i}\right\}=|a|_{x}^{n-1},
\end{aligned}
$$

so that $|a|_{x} \leq 1$, which is a contradiction.

Let $a \in A$ such that $a$ is not integral over $\mathscr{A}$. We show that there exists a prime ideal $\mathfrak{q}$ of $\mathscr{A}$ such that the canonical image of $a$ in $A / S^{-1} \mathfrak{q}$ is not integral over $\mathscr{A} / \mathfrak{q}$. In fact, since $A$ is a $k$-algebra of finite type, it is a noetherian ring. In particular, it admits only finitely many minimal prime ideals $S^{-1} \mathfrak{p}_{1}, \ldots, S^{-1} \mathfrak{p}_{n}$, where $\mathfrak{p}_{1}, \ldots, \mathfrak{p}_{n}$ are prime ideals of $\mathscr{A}$ which do not intersect $S=\mathfrak{o}_{k} \backslash\{0\}$. Assume that, for any $i \in\{1, \ldots, n\}, f_{i}$ is a monic polynomial in $\left(\mathscr{A} / \mathfrak{p}_{i}\right)[T]$ such that $f_{i}\left(\lambda_{i}\right)=0$, where $\lambda_{i}$ is the class of $a$ in $A / S^{-1}\left(\mathfrak{p}_{i}\right)$. Let $F_{i}$ be a monic polynomial in $\mathscr{A}[T]$ whose reduction modulo $\mathfrak{p}_{i}[T]$ coincides with $f_{i}$. One has $F_{i}(a) \in S^{-1} \mathfrak{p}_{i}$ for any $i \in\{1, \ldots, n\}$. Let $F$ be the product of the polynomials $F_{1}, \ldots, F_{n}$. Then $F(a)$ belongs to the intersection $\bigcap_{i=1}^{n} S^{-1} \mathfrak{p}_{i}$, hence is nilpotent, which implies that $a$ is integral over $\mathscr{A}$. To show that there exists $x \in \operatorname{Spec}(A)_{\mathscr{A}}^{\text {an }}$ such that $|a|_{x}>1$ we may replace $\mathscr{A}($ resp. $A)$ by $\mathscr{A} / \mathfrak{q}$ (resp. $\left.A / S^{-1} \mathfrak{q}\right)$ and hence assume that $\mathscr{A}$ is an integral domain without loss of generality.

We set $b=a^{-1}$. Let us see that

$$
b \mathscr{A}[b] \cap \mathfrak{o}_{k} \neq\{0\} \quad \text { and } 1 \notin b \mathscr{A}[b] .
$$

We set $a=a^{\prime} / s$ for some $a^{\prime} \in \mathscr{A}$ and $s \in S$. Then $s=b a^{\prime} \in b \mathscr{A}[b] \cap \mathfrak{o}_{k}$, so that $b \mathscr{A}[b] \cap \mathfrak{o}_{k} \neq\{0\}$. Next we assume that $1 \in b \mathscr{A}[b]$. Then

$$
1=a_{1}^{\prime} b+a_{2}^{\prime} b^{2}+\cdots+a_{n^{\prime}}^{\prime} b^{n^{\prime}}
$$

for some $a_{1}^{\prime}, \ldots, a_{n^{\prime}}^{\prime} \in \mathscr{A}$, so that $a^{n^{\prime}}=a_{1}^{\prime} a^{n^{\prime}-1}+\cdots+a_{n^{\prime}}^{\prime}$, which is a contradiction.

Let $\mathfrak{p}$ be the maximal ideal of $\mathscr{A}[b]$ such that $b \mathscr{A}[b] \subseteq \mathfrak{p}$. As $\mathfrak{p} \cap \mathfrak{o}_{k} \neq\{0\}$ and $\mathfrak{p} \cap \mathfrak{o}_{k} \subseteq \mathfrak{m}_{k}$, we have $\mathfrak{p} \cap \mathfrak{o}_{k}=\mathfrak{m}_{k}$, and hence $\mathfrak{p} \in \operatorname{Spec}(\mathscr{A}[b])_{0}$. Note that $\mathscr{A}[b]$ is finitely generated over $\mathfrak{o}_{k}$ and $\mathscr{A}[b] \otimes_{\mathfrak{o}_{k}} k=A[b]$. Thus, since the reduction map

$$
r_{\mathscr{A}[b]}: \operatorname{Spec}(A[b])_{\mathscr{A}[b]}^{\mathrm{an}} \rightarrow \operatorname{Spec}(\mathscr{A}[b]) \text { 。 }
$$

is surjective, there is $x \in \operatorname{Spec}(A[b])_{\mathscr{A}[b]}^{\text {an }}$ such that $r_{\mathscr{A}[b]}(x)=\mathfrak{p}$. Clearly $x \in$ $\operatorname{Spec}(A)_{\mathscr{A}}^{\text {an }}$. As $b \in \mathfrak{p}$, we have $|b|_{x}<1$, so that $|a|_{x}>1$ because $a b=1$. Therefore,

$$
a \notin \bigcap_{x \in \operatorname{Spec}(A)_{\mathscr{A}}^{\mathrm{an}}}(A,|\cdot| x)_{\leq 1}
$$

as required.

We assume that $X$ is projective. Let $\mathscr{X} \rightarrow \operatorname{Spec}\left(\mathfrak{o}_{k}\right)$ be a flat and projective scheme over Spec $\mathfrak{o}_{k}$ such that the generic fiber of $\mathscr{X} \rightarrow \operatorname{Spec}\left(\mathfrak{o}_{k}\right)$ is $X$. Let $\mathscr{L}$ be an invertible sheaf on $\mathscr{X}$ such that $\left.\mathscr{L}\right|_{X}=L$. We set $h:=\left\{|\cdot|_{\mathscr{L}}(x)\right\}_{x \in X^{\text {an }}}$. For the definition of the metric $|\cdot| \mathscr{L}(x)$ at $x$, see \$1.1.6

Corollary 2.2. Fix $l \in H^{0}(X, L)$. If $|l|_{\mathscr{L}}(x) \leq 1$ for all $x \in X^{\text {an }}$, then there is $s \in \mathfrak{o}_{k} \backslash\{0\}$ such that $s l^{\otimes n} \in H^{0}\left(\mathscr{X}, \mathscr{L}^{\otimes n}\right)$ for all $n \geq 0$. 
Proof. Let $\mathscr{X}=\bigcup_{i=1}^{N} \operatorname{Spec}\left(\mathscr{A}_{i}\right)$ be an affine open covering of $\mathscr{X}$ with the following properties:

(1) $\mathscr{A}_{i}$ is a finitely generated algebra over $\mathfrak{o}_{k}$ for every $i$.

(2) $\operatorname{Spec}\left(\mathscr{A}_{i}\right)_{\circ} \neq \varnothing$ for all $i$.

(3) There is a basis $\omega_{i}$ of $\mathscr{L}$ over $\operatorname{Spec}\left(\mathscr{A}_{i}\right)$ for every $i$.

We set $l=a_{i} \omega_{i}$ for some $a_{i} \in A_{i}:=\mathscr{A}_{i} \otimes_{\mathfrak{o}_{k}} k$. By our assumption, $\left|a_{i}\right|_{x} \leq 1$ for all $x \in \operatorname{Spec}\left(A_{i}\right)_{\mathscr{A}_{i}}^{\text {an }}$ Therefore, by Theorem 2.1] $a_{i}$ is integral over $\mathscr{A}_{i}$, so that, by the following Lemma 2.3, we can find $s_{i} \in S$ such that $s_{i} a_{i}^{n} \in \mathscr{A}_{i}$ for all $n \geq 0$. We set $s=s_{1} \cdots s_{N}$. Then, as $s a_{i}^{n} \in \mathscr{A}_{i}$ for all $n \geq 0$ and $i=1, \ldots, N$, we have the assertion.

Lemma 2.3. Let $A$ be a commutative ring and $S$ a multiplicatively closed subset of $A$, which consists of regular elements of $A$. If $t \in S^{-1} A$ and $t$ is integral over $A$, then there is $s \in S$ such that $s t^{n} \in A$ for all $n \geq 0$.

Proof. As $t$ is integral over $A$, there are $a_{1}, \ldots, a_{r-1} \in A$ such that

$$
t^{r}=a_{1} t^{r-1}+\cdots+a_{r-1} t+a_{r}
$$

We choose $s \in S$ such that $s t^{i} \in A$ for $i=0, \ldots, r-1$. By induction on $n$, we prove that $s t^{n} \in A$ for all $n \geq 0$. Note that

$$
t^{n}=a_{1} t^{n-1}+\cdots+a_{r-1} t^{n-r+1}+a_{r} t^{n-r} .
$$

Thus, if $s t^{i} \in A$ for $i=0, \ldots, n-1$, then $s t^{n} \in A$ because

$$
s t^{n}=a_{1}\left(s t^{n-1}\right)+\cdots+a_{r-1}\left(s t^{n-r+1}\right)+a_{r}\left(s t^{n-r}\right) .
$$

\section{Continuous Metrics OF INVERTIBLE SHEAVES}

In this section, we consider several properties of continuous metrics of invertible sheaves. Let $h=\left\{|\cdot|_{h}(x)\right\}_{x \in X^{\text {an }}}$ and $h^{\prime}=\left\{|\cdot|_{h^{\prime}}(x)\right\}_{x \in X^{\text {an }}}$ be continuous metrics of $L^{\text {an }}$ (cf. \$1.1.4). As $L(x):=L \otimes_{\mathscr{O}_{X}} \hat{\kappa}(x)$ is a 1-dimensional vector space over $\hat{\kappa}(x), h+h^{\prime}:=\left\{|\cdot|_{h}(x)+|\cdot|_{h^{\prime}}(x)\right\}_{x \in X^{a n}}$ forms a continuous metric of $L^{\text {an }}$. Indeed, we can find a continuous positive function $\varphi$ on $X^{\text {an }}$ such that $|\cdot|_{h^{\prime}}(x)=\varphi(x)|\cdot|_{h}(x)$ for any $x \in X^{\text {an }}$. Thus

$$
h+h^{\prime}=\left\{(1+\varphi(x))|\cdot|_{h}(x)\right\}_{x \in X^{\text {an }}}
$$

is a continuous metric of $L^{\text {an }}$.

Lemma 3.1. There is a continuous metric of $L^{\text {an }}$.

Proof. Let us choose an affine open covering $X=\bigcup_{i=1}^{N} U_{i}$ together with a local basis $\omega_{i}$ of $L$ on each $U_{i}$. Let $h_{i}$ be a metric of $L^{\text {an }}$ over $U_{i}^{\text {an }}$ given by $\left|\omega_{i}\right|_{h_{i}}(x)=$ 1 for $x \in U_{i}^{\text {an }}$. As $X^{\text {an }}$ is paracompact (locally compact and $\sigma$-compact), we can find a partition of unity $\left\{\rho_{i}\right\}_{i=1, \ldots, N}$ of continuous functions on $X^{\text {an }}$ such that $\operatorname{supp}\left(\rho_{i}\right) \subseteq U_{i}^{\text {an }}$ for all $i$. If we set $|\cdot|_{h}(x)=\sum_{i=1}^{N} \rho_{i}(x)|\cdot|_{h_{i}}(x)$, then $h=$ $\left\{|\cdot|_{h}(x)\right\}_{x \in X^{\text {an }}}$ yields a continuous metric of $L^{\text {an }}$. 
3.1. Extension theorem for a metric arising from a model. We assume that $X$ is

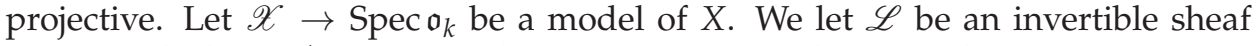
on $\mathscr{X}$ such that $\left.\mathscr{L}\right|_{X}=L$. We have seen in $\$ 1.1 .6$ that $\mathscr{L}$ induces a continuous metric $h=\{|\cdot| \mathscr{L}(x)\}_{x \in X^{\text {an }}}$ of $L^{\text {an }}$.

Theorem 3.2. We assume that $|\cdot|$ is non-trivial and $\mathscr{L}$ is an ample invertible sheaf. Fix a closed subscheme $Y$ of $X, l \in H^{0}\left(Y,\left.L\right|_{Y}\right)$ and a positive number $\epsilon$. Then there are a positive integer $n$ and $s \in H^{0}\left(X, L^{\otimes n}\right)$ such that $\left.s\right|_{Y}=l^{\otimes n}$ and

$$
\|s\|_{h^{n}} \leq e^{n \epsilon}\left(\|l\|_{Y, h}\right)^{n} .
$$

Proof. Clearly, we may assume that $l \neq 0$. Let $\mathscr{Y}$ be the Zariski closure of $Y$ in $\mathscr{X}$ (cf. \$1.1.7).

Claim 3.2.1. There are a positive integer $a$ and $\alpha \in k^{\times}$such that

$$
e^{-a \epsilon / 2} \leq\left\|\alpha l^{\otimes a}\right\|_{Y, h^{a}} \leq 1 .
$$

Proof. First we assume that $|\cdot|$ is discrete. We take a positive integer $a$ such that $e^{-\epsilon a / 2} \leq|\omega|$. We also choose $\alpha \in k^{\times}$such that

$$
\left|\alpha^{-1}\right|=\min \left\{|\gamma| \mid \gamma \in k^{\times} \text {and }\left\|l^{\otimes a}\right\|_{Y, h^{a}} \leq|\gamma|\right\} .
$$

Then, as $\left\|l^{\otimes a}\right\|_{Y, h^{a}} \leq\left|\alpha^{-1}\right| \leq \mid \mathcal{O}^{-1}\left\|l^{\otimes a}\right\|_{Y, h^{a}}$, we have

$$
e^{-a \epsilon / 2} \leq|\omega| \leq\left\|\alpha l^{\otimes a}\right\|_{Y, h^{a}} \leq 1 .
$$

Next we assume that $|\cdot|$ is not discrete. In this case, $\left|k^{\times}\right|$is dense in $\mathbb{R}_{>0}$ by Lemma 1.18 , so that we can choose $\beta \in k^{\times}$such that

$$
e^{-\epsilon / 2} \leq\|l\|_{Y, h} /|\beta| \leq 1
$$

Thus if we set $\alpha=\beta^{-1}$ and $a=1$, we have the assertion.

By Corollary 2.2, there is $\beta \in \mathfrak{o}_{K} \backslash\{0\}$ such that

$$
\beta\left(\alpha l^{\otimes a}\right)^{\otimes m} \in H^{0}\left(\mathscr{Y},\left.\mathscr{L}^{\otimes a m}\right|_{\mathscr{Y}}\right)
$$

for all $m \geq 0$. We choose a positive integer $m$ such that $|\beta|^{-1} \leq e^{a m \epsilon / 2}$ and

$$
H^{0}\left(\mathscr{X}, \mathscr{L}^{\otimes a m}\right) \rightarrow H^{0}\left(\mathscr{Y},\left.\mathscr{L}^{\otimes a m}\right|_{\mathscr{Y}}\right)
$$

is surjective, so that we can find $l_{m} \in H^{0}\left(\mathscr{X}, \mathscr{L}^{\otimes a m}\right)$ such that $\left.l_{m}\right|_{\mathscr{Y}}=\beta\left(\alpha l^{\otimes a}\right)^{\otimes m}$. Note that $\left\|l_{m}\right\|_{h^{a m}} \leq 1$. Thus, if we set $s=\beta^{-1} \alpha^{-m} l_{m}$, then $\left.s\right|_{\mathscr{Y}}=l^{\otimes a m}$ and

$$
\begin{aligned}
\|s\|_{h^{a m}} & =|\beta|^{-1}|\alpha|^{-m}\left\|l_{m}\right\|_{h^{a m}} \leq e^{a m \epsilon / 2}|\alpha|^{-m} \\
& \leq e^{a m \epsilon / 2}|\alpha|^{-m}\left(e^{a \epsilon / 2}\left\|\alpha l^{\otimes a}\right\|_{Y, h^{a}}\right)^{m}=e^{a m \epsilon}\left(\|l\|_{Y, h}\right)^{a m},
\end{aligned}
$$

as required. 
3.2. Quotient metric. Let $V$ be a finite-dimensional vector space over $k$. We assume that there is a surjective homomorphism

$$
\pi: V \otimes_{k} \mathscr{O}_{X} \rightarrow L \text {. }
$$

For each $e \in V, \pi(e \otimes 1)$ yields a global section of $L$, that is, $\pi(e \otimes 1) \in H^{0}(X, L)$. We denote it by $\tilde{e}$. Let $\|\cdot\|$ be a norm of $V$ and $\bar{V}:=(V,\|\cdot\|)$. Let $\|\cdot\|_{\hat{\kappa}(x)}$ be a norm of $V \otimes_{k} \hat{\kappa}(x)$ obtained by the scalar extension of $\|\cdot\|$ (cf. Definition 1.11). Let $|\cdot| \frac{q u o t}{V}(x)$ be the quotient norm of $L(x):=L \otimes \hat{\kappa}(x)$ induced by $\|\cdot\|_{\hat{\kappa}(x)}$ and the surjective homomorphism $V \otimes_{k} \hat{\kappa}(x) \rightarrow L(x)$.

Lemma 3.3. Let $h$ be a continuous metric of $L^{\text {an }}\left(c f\right.$. Lemma 3.1). Let $\left(e_{0}, \ldots, e_{r}\right)$ be an orthogonal basis of $V$ with respect to $\|\cdot\|$. Then, for $s \in H^{0}(X, L)$,

on $X^{\text {an }}$.

$$
|s| \frac{\text { quot }}{V}(x)=\frac{|s|_{h}(x)}{\max _{i=0, \ldots, r}\left\{\frac{\left|\tilde{e}_{i}\right|_{h}(x)}{\left\|e_{i}\right\|}\right\}}
$$

Proof. We set $I:=\left\{i \mid \tilde{e}_{i} \neq 0\right.$ in $\left.H^{0}(X, L)\right\}$ and $U_{i}:=\left\{p \in X \mid \tilde{e}_{i} \neq 0\right.$ at $\left.p\right\}$ for $i \in I$.

Claim 3.3.1. For a fixed $j \in I$, if we set $\tilde{e}_{i}=a_{i j} \tilde{e}_{j}$ on $U_{j}\left(a_{i j} \in \mathscr{O}_{U_{j}}\right)$, then

$$
\left|\tilde{e}_{j}\right| \frac{\text { quot }}{V}(x)=\frac{1}{\max _{i=0, \ldots, r}\left\{\frac{\left|a_{i j}\right|_{x}}{\left\|e_{i}\right\|}\right\}}
$$

on $U_{j}^{\text {an }}$.

Proof. We set $c_{i}=\left\|e_{i}\right\|$ for $i=0, \ldots, r$. Without loss of generality, we may assume that $j=0$, that is, we need to show that

Since

$$
\left|\tilde{e}_{0}\right| \frac{\text { quot }}{V}(x)=\frac{1}{\max \left\{1 / c_{0},\left|a_{10}\right|_{x} / c_{1}, \ldots,\left|a_{r 0}\right|_{x} / c_{r}\right\}} .
$$

$$
\operatorname{ker}\left(\pi_{x}: V \otimes_{k} \hat{\kappa}(x) \rightarrow L \otimes_{\mathscr{O}_{X}} \hat{\kappa}(x)\right)=\left\langle e_{1}-a_{10}(x) e_{0}, \ldots, e_{r}-a_{r 0}(x) e_{0}\right\rangle
$$

for $x \in U_{0}^{\mathrm{an}}$, we have

$$
\left|\tilde{e}_{0}\right| \frac{\text { quot }}{V}(x)=\inf \left\{f\left(\lambda_{1}, \ldots, \lambda_{r}\right) \mid\left(\lambda_{1}, \ldots, \lambda_{r}\right) \in \hat{\kappa}(x)^{r}\right\},
$$

where $f\left(\lambda_{1}, \ldots, \lambda_{r}\right):=\left\|e_{0}+\sum_{i=1}^{r} \lambda_{i}\left(e_{i}-a_{i 0}(x) e_{0}\right)\right\|_{\hat{\kappa}(x)}$. Note that

$$
f\left(\lambda_{1}, \ldots, \lambda_{r}\right)=\max \left\{c_{0}\left|1-\sum_{i=1}^{r} \lambda_{i} a_{i 0}(x)\right|_{x}, c_{1}\left|\lambda_{1}\right|_{x}, \ldots, c_{r}\left|\lambda_{r}\right| x\right\} .
$$

As

$$
\max \left\{\alpha_{0}, \ldots, \alpha_{r}\right\} \max \left\{\beta_{0}, \ldots, \beta_{r}\right\} \geq \max \left\{\alpha_{0} \beta_{0}, \ldots, \alpha_{r} \beta_{r}\right\}
$$

for $\alpha_{0}, \ldots, \alpha_{r}, \beta_{0}, \ldots, \beta_{r} \in \mathbb{R}_{\geq 0}$, we have

$$
\begin{gathered}
f\left(\lambda_{1}, \ldots, \lambda_{r}\right) \cdot \max \left\{1 / c_{0},\left.\left|a_{10}(x)\right|\right|_{x} / c_{1}, \ldots,\left.\left|a_{r 0}(x)\right|\right|_{x} / c_{r}\right\} \\
\geq \max \left\{\left|1-\sum_{i=1}^{r} \lambda_{i} a_{i 0}(x)\right|_{x},\left|\lambda_{1} a_{10}(x)\right|_{x}, \ldots,\left|\lambda_{r} a_{r 0}(x)\right|_{x}\right\} \\
\geq\left|1-\sum_{i=1}^{r} \lambda_{i} a_{i 0}(x)+\sum_{i=1}^{r} \lambda_{i} a_{i 0}(x)\right|_{x}=1 .
\end{gathered}
$$


Therefore, we obtain

$\inf \left\{f\left(\lambda_{1}, \ldots, \lambda_{r}\right) \mid\left(\lambda_{1}, \ldots, \lambda_{r}\right) \in \hat{\kappa}(x)^{r}\right\} \geq \frac{1}{\max \left\{1 / c_{0},\left|a_{10}(x)\right|_{x} / c_{1}, \ldots,\left|a_{r 0}(x)\right|{ }_{x} / c_{r}\right\}}$.

We need to see that

$$
f\left(\eta_{1}, \ldots, \eta_{r}\right)=\frac{1}{\max \left\{1 / c_{0},\left|a_{10}(x)\right|_{x} / c_{1}, \ldots,\left|a_{r 0}(x)\right|_{x} / c_{r}\right\}} .
$$

for some $\eta_{1}, \ldots, \eta_{r} \in \hat{\kappa}(x)$. As $f(0, \ldots, 0)=c_{0}$, the assertion holds if

$$
\max \left\{1 / c_{0},\left|a_{10}(x)\right|_{x} / c_{1}, \ldots,\left|a_{r 0}(x)\right|_{x} / c_{r}\right\}=1 / c_{0} .
$$

Next we assume that

$$
\max \left\{1 / c_{0},\left|a_{10}(x)\right|_{x} / c_{1}, \ldots,\left|a_{r 0}(x)\right|_{x} / c_{r}\right\}=\left|a_{i 0}(x)\right|_{x} / c_{i}
$$

for some $i$. Clearly $a_{i 0}(x) \neq 0$. If we set

$$
\eta_{j}= \begin{cases}0 & \text { if } j \neq i, \\ 1 / a_{i 0}(x) & \text { if } j=i,\end{cases}
$$

then $f\left(\eta_{1}, \ldots, \eta_{r}\right)=c_{i} /\left|a_{i 0}(x)\right|_{x}$, as required.

If we set $s=f \tilde{e}_{j}$ on $U_{j}\left(f \in \mathscr{O}_{U_{j}}\right)$, then $|s| \frac{q u o t}{V}(x)=\left.|f|_{x}\left|\tilde{e}_{j}\right|\right|_{V} ^{\text {quot }}(x)$ on $U_{j}^{\text {an }}$, so that, by Claim 3.3.1.

$$
|s| \frac{q_{V}^{\text {uoot }}}{V}(x)=\frac{|f|_{x}}{\max _{i=0, \ldots, r}\left\{\frac{\left|a_{i j}\right|_{x}}{\left\|e_{i}\right\|}\right\}} .
$$

On the other hand, $|s|_{h}(x)=|f|_{x}\left|\tilde{e}_{j}\right|_{h}(x)$ and $\left|\tilde{e}_{i}\right|_{h}(x)=\left|a_{i j}\right|_{x}\left|\tilde{e}_{j}\right|_{h}(x)$ for $i=$ $0, \ldots, r$. Thus

$$
|s| \frac{\text { quot }}{V}(x)=\frac{|s|_{h}(x)}{\max _{i=0, \ldots, r}\left\{\frac{\left|\tilde{e}_{i}\right|_{h}(x)}{\| e_{i} \mid}\right\}}
$$

on $U_{j}^{\text {an }}$. Therefore, the assertion follows because $X=\bigcup_{j \in I} U_{j}$.

Corollary 3.4. $\left\{|\cdot| \frac{q u o t}{V}(x)\right\}_{x \in X^{\text {an }}}$ yields a continuous metric of $L^{\text {an }}$.

Proof. If $V$ has an orthogonal basis with respect to $\|\cdot\|$, then the assertion follows from Lemma 3.3

In general, by Proposition 1.3 , for each $n \in \mathbb{Z}_{>0}$, we choose a basis

$$
\left(e_{n, 0}, e_{n, 1}, \ldots, e_{n, r}\right)
$$

of $V$ such that

$$
(1-1 / n) \max \left\{\left|c_{0}\right|\left\|e_{n, 0}\right\|, \ldots,\left|c_{r}\right|\left\|e_{n, r}\right\|\right\} \leq\left\|c_{0} e_{n, 0}+\cdots+c_{r} e_{n, r}\right\|
$$

for all $c_{0}, \ldots, c_{r} \in k$. If we set

$$
\left\|c_{0} e_{n, 0}+\cdots+c_{r} e_{n, r}\right\|_{n}:=\max \left\{\left|c_{0}\right|\left\|e_{n, 0}\right\|, \ldots,\left|c_{r}\right|\left\|e_{n, r}\right\|\right\}
$$

for $c_{0}, \ldots, c_{r} \in k$. Then $(1-1 / n)\|\cdot\|_{n} \leq\|\cdot\| \leq\|\cdot\|_{n}$, so that

$$
(1-1 / n)|\cdot|_{\left(V,\|\cdot\|_{n}\right)}^{\text {quot }}(x) \leq|\cdot|_{(V,\|\cdot\|)}^{\text {quot }}(x) \leq|\cdot|_{\left(V,\|\cdot\|_{n}\right)}^{\text {quot }}(x)
$$


for all $x \in X^{\text {an }}$. Let $\omega$ be a local basis of $L$ over an open set $U$. Then the above inequalities imply that

$$
\log (1-1 / n) \leq \log \left(|\omega|_{(V,\|\cdot\|)}^{\text {quot }}(x)\right)-\log \left(|\omega|_{(V,\|\cdot\| n)}^{\text {quot }}(x)\right) \leq 0
$$

for all $x \in U^{\text {an }}$, which shows that the sequence

$$
\left\{\log \left(|\omega|_{\left(V,\|\cdot\|_{n}\right)}^{\text {quot }}(x)\right)\right\}_{n=1}^{\infty}
$$

converges to $\log \left(|\omega|_{(V,\|\cdot\|)}^{\text {quot }}(x)\right)$ uniformly on $U^{\text {an }}$. Thus, by the previous observation,

$$
\log \left(|\omega|_{(V,\|\cdot\|)}^{\text {quot }}(x)\right)
$$

is continuous on $U^{\text {an }}$.

From now on and until the end of the subsection, we assume that $X$ is projective and $L$ is generated by global sections. Let $h=\left\{|\cdot|_{h}(x)\right\}_{x \in X}$ an be a continuous metric of $L^{\text {an }}$. As $H^{0}(X, L) \otimes_{k} \mathscr{O}_{X} \rightarrow L$ is surjective, by Corollary 3.4 .

$$
h^{\text {quot }}=\left\{|\cdot|_{\left(H^{0}(X, L),\|\cdot\|_{h}\right)}^{\text {quot }}(x)\right\}_{x \in X^{\text {an }}}
$$

yields a continuous metric of $L^{\text {an }}$. For simplicity, we denote $|\cdot|_{\left(H^{0}(X, L),\|\cdot\|_{h}\right)}^{\text {quot }}(x)$ by $|\cdot|_{h}^{\text {quot }}(x)$. Moreover, the supreme norm of $H^{0}(X, L)$ arising from $h^{\text {quot }}$ is denoted by $\|\cdot\|_{h}^{\text {quot }}$, that is, $\|\cdot\|_{h}^{\text {quot }}:=\|\cdot\|_{h}$ quot.

Lemma 3.5. (1) $|\cdot|_{h}(x) \leq|\cdot|_{h}^{\text {quot }}(x)$ for all $x \in X^{\text {an }}$.

(2) $\|\cdot\|_{h}=\|\cdot\|_{h}^{\text {quot }}$.

(3) Let $\left(L^{\prime}, h^{\prime}\right)$ be a pair of an invertible sheaf $L^{\prime}$ on $X$ and a continuous metric

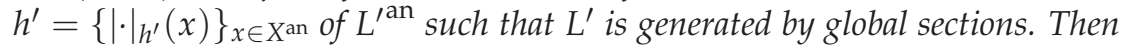

$$
\left|l \cdot l^{\prime}\right|_{h \otimes h^{\prime}}^{\text {quot }}(x) \leq|l|_{h}^{\text {quot }}(x)\left|l^{\prime}\right|_{h^{\prime}}^{\text {quot }}(x)
$$

for $l \in L(x)$ and $l^{\prime} \in L^{\prime}(x)$.

Proof. (1) Fix $l \in L(x) \backslash\{0\}$. For $\epsilon>0$, let $\left(e_{1}, \ldots, e_{n}\right)$ be an $e^{-\epsilon}$-orthogonal basis of $H^{0}(X, L)$ with respect to $\|\cdot\|_{h}$. There is $s \in H^{0}(X, L) \otimes_{k} \hat{\kappa}(x)$ such that $s(x)=l$ and $\|s\|_{h, \hat{\kappa}(x)} \leq e^{\epsilon}|l|_{h}^{\text {quot }}(x)$. We set $s=a_{1} e_{1}+\cdots+a_{n} e_{n}\left(a_{1}, \ldots, a_{n} \in \hat{\kappa}(x)\right)$. Then, by Proposition 1.12

$$
\begin{aligned}
\|S\|_{h, \hat{\kappa}(x)} & \geq e^{-\epsilon} \max \left\{\left|a_{1}\right| x|| e_{1}\left\|_{h}, \ldots,\left|a_{n}\right|_{x}\right\| e_{n} \|_{h}\right\} \\
& \geq e^{-\epsilon} \max \left\{\left|a_{1}\right| x\left|e_{1}\right|_{h}(x), \ldots,\left|a_{n}\right|_{x}\left|e_{n}\right|_{h}(x)\right\} \geq e^{-\epsilon}|l|_{h}(x),
\end{aligned}
$$

so that $|l|_{h}(x) \leq e^{2 \epsilon}|l|_{h}^{\text {quot }}(x)$, and hence the assertion follows because $\epsilon$ is an arbitrary positive number.

(2) By (1), we have $\|\cdot\|_{h} \leq\|\cdot\|_{h}^{\text {quot }}$. On the other hand, as $|s|_{h}^{\text {quot }}(x) \leq\|s\|_{h}$ for $s \in H^{0}(X, L)$, we have $\|s\|_{h}^{\text {quot }} \leq\|s\|_{h}$.

(3) For $\epsilon>0$, there are $s \in H^{0}(X, L) \otimes_{k} \hat{\kappa}(x)$ and $s^{\prime} \in H^{0}\left(X, L^{\prime}\right) \otimes_{k} \hat{\kappa}(x)$ such that

$$
s(x)=l, s^{\prime}(x)=l^{\prime},\|s\|_{h, \hat{\kappa}(x)} \leq e^{\epsilon}|l|_{h}^{\text {quot }}(x) \text { and }\left\|s^{\prime}\right\|_{h^{\prime}, \hat{\kappa}(x)} \leq e^{\epsilon}\left|l^{\prime}\right|_{h^{\prime}}^{\text {quot }}(x) .
$$


Here let us see that $\left\|s \cdot s^{\prime}\right\|_{h \otimes h^{\prime}, \hat{\kappa}(x)} \leq e^{2 \epsilon}\|s\|_{h, \hat{\kappa}(x)}\left\|s^{\prime}\right\|_{h^{\prime}, \hat{\kappa}(x)}$. Let $\left(s_{1}, \ldots, s_{m}\right)$ and $\left(s_{1}^{\prime}, \ldots, s_{m^{\prime}}^{\prime}\right)$ be $e^{-\epsilon}$-orthogonal bases of $H^{0}(X, L)$ and $H^{0}\left(X, L^{\prime}\right)$, respectively. If we set $s=t_{1} s_{1}+\cdots+t_{m} s_{m}$ and $s^{\prime}=t_{1}^{\prime} s_{1}^{\prime}+\cdots+t_{m^{\prime}}^{\prime} s_{m^{\prime}}^{\prime}\left(t_{1}, \ldots, t_{m}, t_{1}^{\prime}, \ldots, t_{m^{\prime}}^{\prime} \in\right.$ $\hat{\kappa}(x))$, then

$$
s \cdot s^{\prime}=\sum_{i, j} t_{i} t_{j}^{\prime} s_{i} \cdot s_{j}^{\prime}
$$

Thus,

$$
\begin{aligned}
\left\|s \cdot s^{\prime}\right\|_{h \otimes h^{\prime}, \hat{\kappa}(x)} & \leq \max _{i, j}\left\{\left|t_{i}\right|_{x}\left|t_{j}^{\prime}\right|_{x}\left\|s_{i} \cdot s_{j}^{\prime}\right\|_{h \otimes h^{\prime}}\right\} \leq \max _{i, j}\left\{\left|t_{i}\right|_{x}\left|t_{j}^{\prime}\right|_{x}\left\|s_{i}\right\|_{h}\left\|s_{j}^{\prime}\right\|_{h^{\prime}}\right\} \\
& \leq \max _{i}\left\{\left|t_{i}\right|_{x}\left\|s_{i}\right\|_{h}\right\} \max _{j}\left\{\left|t_{j}^{\prime}\right|_{x}\left\|s_{j}^{\prime}\right\|_{h^{\prime}}\right\} \\
& \leq e^{2 \epsilon}\|s\|_{h, \hat{\kappa}(x)}\left\|s^{\prime}\right\|_{h^{\prime}, \hat{\kappa}(x)} .
\end{aligned}
$$

Therefore, we have $\left(s \cdot s^{\prime}\right)(x)=l \cdot l^{\prime}$ and

$$
\left|l \cdot l^{\prime}\right|_{h \otimes h^{\prime}}^{\text {quot }}(x) \leq\left\|s \cdot s^{\prime}\right\|_{h \otimes h^{\prime}, \hat{\kappa}(x)} \leq e^{2 \epsilon}\|s\|_{h, \hat{\kappa}(x)}\left\|s^{\prime}\right\|_{h^{\prime}, \hat{\kappa}(x)} \leq e^{4 \epsilon}|l|_{h}^{\text {quot }}(x)\left|l^{\prime}\right|_{h^{\prime}}^{\text {quot }}(x),
$$

as required.

Proposition 3.6. If there are a normed finite-dimensional vector space $(V,\|\cdot\|)$ and a surjective homomorphism $V \otimes_{k} \mathscr{O}_{X} \rightarrow L$ such that $h$ is given by $\left\{\left.|\cdot|\right|_{(V,\|\cdot\|)} ^{\text {quot }}(x)\right\}_{x \in X^{\text {an' }}}$, then $|\cdot|_{h^{n}}(x)=|\cdot|_{h^{n}}^{\text {quot }}(x)$ for all $n \geq 1$.

Proof. First we consider the case $n=1$. Fix $l \in L(x) \backslash\{0\}$. For $\epsilon>0$, there is $s \in V \otimes_{k} \hat{\kappa}(x)$ such that $\tilde{s}(x)=l$ and $\|s\|_{\hat{\kappa}(x)} \leq e^{\epsilon}|l|_{h}(x)$.

Note that $\|\tilde{e}\|_{h} \leq\|e\|$ for all $e \in V$. Let $\left(e_{1}, \ldots, e_{r}\right)$ be an $e^{-\epsilon}$-orthogonal basis of $V$ with respect to $\|\cdot\|$. If we set $s=a_{1} e_{1}+\cdots+a_{r} e_{r}\left(a_{1}, \ldots, a_{r} \in \hat{\kappa}(x)\right)$, then, by Proposition 1.12

$$
\begin{aligned}
\|\tilde{S}\|_{h, \hat{\kappa}(x)} & \leq \max \left\{\left.\left|a_{1}\right|\right|_{x}\left\|\tilde{e}_{1}\right\|_{h}, \ldots,\left|a_{r}\right|_{x}\left\|\tilde{e}_{r}\right\|_{h}\right\} \\
& \leq \max \left\{\left|a_{1}\right|_{x}\left\|e_{1}\right\|, \ldots,\left|a_{r}\right|_{x}\left\|e_{r}\right\|\right\} \\
& \leq e^{\epsilon}\|s\|_{\hat{\kappa}(x)}
\end{aligned}
$$

so that

$$
|l|_{h}^{\text {quot }}(x) \leq\|\tilde{s}\|_{h, \hat{\kappa}(x)} \leq e^{\epsilon}\|s\|_{\hat{\kappa}(x)} \leq e^{2 \epsilon}|l|_{h}(x),
$$

and hence $|l|_{h}^{\text {quot }}(x) \leq|l|_{h}(x)$ by taking $\epsilon \rightarrow 0$. Thus the assertion for $n=1$ follows from (1) in Lemma 3.5 .

In general, by using (3) in Lemma 3.5 .

$$
\left|l^{n}\right|_{h^{n}}(x)=\left(|l|_{h}(x)\right)^{n}=\left(|l|_{h}^{\text {quot }}(x)\right)^{n} \geq\left|l^{n}\right|_{h^{n}}^{\text {quot }}(x),
$$

and hence we have the assertion by (1) in Lemma 3.5

Lemma 3.7. We assume that there are a normed finite-dimensional vector space $(V,\|\cdot\|)$ and a surjective homomorphism $V \otimes_{k} \mathscr{O}_{X} \rightarrow L$ such that $h$ is given by $\left\{|\cdot|_{(V,\|\cdot\|)}^{\text {quot }}(x)\right\}_{x \in X^{\text {an }}}$. Let $k^{\prime}$ be an extension field of $k$, and let $|\cdot|^{\prime}$ be a complete absolute value of $k^{\prime}$ as an extension of $|\cdot|$. We set

$$
X^{\prime}:=X \times_{\operatorname{Spec}(k)} \operatorname{Spec}\left(k^{\prime}\right), \quad L=L \otimes_{k} k^{\prime} \quad \text { and } \quad V^{\prime}:=V \otimes_{k} k^{\prime} .
$$


Let $\|\cdot\|^{\prime}$ be a norm of $V^{\prime}$ obtained by the scalar extension of $\|\cdot\|$. Moreover, let $h^{\prime}$ be a continuous metric of $L^{\prime a n}$ given by the scalar extension of $h$. Then $h^{\prime}$ coincides with $\left\{|\cdot|_{\left(V^{\prime},\|\cdot\|^{\prime}\right)}^{\text {quot }}\left(x^{\prime}\right)\right\}_{x^{\prime} \in X^{\prime a n}}$.

Proof. Let $f: X^{\prime} \rightarrow X$ be the projection. For $x^{\prime} \in X^{\prime a n}$, we set $x=f^{\text {an }}\left(x^{\prime}\right)$. Then $\hat{\kappa}(x) \subseteq \hat{\kappa}\left(x^{\prime}\right)$ and $\left(L \otimes_{k} \hat{\kappa}(x)\right) \otimes_{\hat{\kappa}(x)} \hat{\kappa}\left(x^{\prime}\right)=L^{\prime} \otimes_{k^{\prime}} \hat{\kappa}\left(x^{\prime}\right)$, that is, $L(x) \otimes_{\hat{\kappa}(x)} \hat{\kappa}\left(x^{\prime}\right)=$ $L^{\prime}\left(x^{\prime}\right)$. Moreover, $V^{\prime} \otimes_{k^{\prime}} \hat{\kappa}\left(x^{\prime}\right)=\left(V \otimes_{k} \hat{\kappa}(x)\right) \otimes_{\hat{\kappa}(x)} \hat{\kappa}\left(x^{\prime}\right)$, and by Lemma 1.13 . $\|\cdot\|_{\hat{\kappa}\left(x^{\prime}\right)}^{\prime}=\|\cdot\|_{\hat{\kappa}\left(x^{\prime}\right)}=\|\cdot\|_{\hat{\kappa}(x), \hat{\kappa}\left(x^{\prime}\right)}$. Thus the assertion follows from Lemma 1.14

Proposition 3.8. We assume that there is a subspace $H$ of $H^{0}(X, L)$ such that $H \otimes_{k}$ $\mathscr{O}_{X} \rightarrow L$ is surjective and the morphism $\phi_{H}: X \rightarrow \mathbb{P}(H)$ induced by $H$ is a closed embedding. We identify $X$ with $\phi_{H}(X)$, so that $L=\left.\mathscr{O}_{\mathbb{P}(H)}(1)\right|_{X}$. Let $\|\cdot\|$ be a norm of $H$ such that $H$ has an orthonormal basis $\left(e_{1}, \ldots, e_{r}\right)$ with respect to $\|\cdot\|$. We set

$$
h:=\left\{|\cdot|_{(H,\|\cdot\|)}^{\text {quot }}(x)\right\}_{x \in X^{\text {an }}} \text { and } \quad \mathscr{H}:=\mathfrak{o}_{k} e_{1}+\cdots+\mathfrak{o}_{k} e_{r}=(H,\|\cdot\|)_{\leq 1} .
$$

Let $\mathscr{X}$ be the Zariski closure of $X$ in $\mathbb{P}(\mathscr{H})$ (cf. \$1.1.7) and $\mathscr{L}:=\left.\mathscr{O}_{\mathbb{P}(\mathscr{H})}(1)\right|_{\mathscr{X}}$. Then $|\cdot|_{h}(x)=|\cdot|_{\mathscr{L}}(x)$ for all $x \in X^{\text {an }}$.

Proof. First let us see that $|s|_{h}(x) \leq|s| \mathscr{L}(x)$ for $s \in H$. Let $\omega_{\xi}$ be a local basis of $\mathscr{L}$ at $\xi=r_{\mathscr{X}}(x)$. If we set $s=s_{\xi} \omega_{\xi}$, then

$$
|s|_{\mathscr{L}}(x)=\left|s_{\xi}\right|_{x} .
$$

As $s_{\xi}^{-1} s \in \mathscr{L}_{\xi}$ and $\mathscr{H} \otimes_{\mathfrak{o}_{k}} \mathscr{O}_{\mathscr{X}, \xi} \rightarrow \mathscr{L}_{\xi}$ is surjective, there are $l_{1}, \ldots, l_{r} \in \mathscr{H}$ and $a_{1}, \ldots, a_{r} \in \mathscr{O}_{\mathscr{X}, \xi}$ such that $s_{\xi}^{-1} s=a_{1} l_{1}+\cdots+a_{r} l_{r}$. Therefore,

$$
\begin{aligned}
\left|s_{\xi}^{-1} s\right|_{h}(x) & \leq \max \left\{\left|a_{1} l_{1}\right|_{h}(x), \ldots,\left|a_{r} l_{r}\right|_{h}(x)\right\} \\
& =\max \left\{\left|a_{1}\right|_{x}\left|l_{1}\right|_{h}(x), \ldots,\left|a_{r}\right|_{x}\left|l_{r}\right|_{h}(x)\right\} \leq 1,
\end{aligned}
$$

so that $|s|_{h}(x) \leq\left|s_{\xi}\right|_{x}=|s|_{\mathscr{L}}(x)$, as required.

Next let us see that $|l|_{\mathscr{L}}(x) \leq\|l\|_{\hat{\kappa}(x)}$ for all $l \in H \otimes \hat{\kappa}(x)$. By Proposition 1.12 . $\left(e_{1}, \ldots, e_{r}\right)$ is an orthonormal basis of $H \otimes \hat{\kappa}(x)$ with respect to $\|\cdot\|_{\hat{\kappa}(x)}$. Thus, if we set $l=a_{1} e_{1}+\cdots+a_{r} e_{r}\left(a_{1}, \ldots, a_{r} \in \hat{\kappa}(x)\right)$, then

$$
\begin{aligned}
|l|_{\mathscr{L}}(x) & \leq \max \left\{\left|a_{1}\right|_{x}\left|e_{1}\right| \mathscr{L}(x), \ldots,\left|a_{r}\right|_{x}\left|e_{r}\right| \mathscr{L}(x)\right\} \\
& \leq \max \left\{\left|a_{1}\right|_{x}, \ldots,\left|a_{r}\right|_{x}\right\}=\|l\|_{\hat{\kappa}(x)} .
\end{aligned}
$$

Finally let us see that $|s| \mathscr{L}(x) \leq|s|_{h}(x)$ for $s \in H$. For $\epsilon>0$, we choose $l \in H \otimes \hat{\kappa}(x)$ such that $l(x)=s(x)$ and $\|l\|_{\hat{\kappa}(x)} \leq e^{\epsilon}|s|_{h}(x)$. Then, by the previous observation,

$$
|s| \mathscr{L}(x)=|l|_{\mathscr{L}}(x) \leq\|l\|_{\hat{\kappa}(x)} \leq e^{\epsilon}|s|_{h}(x) .
$$

Thus the assertion follows.

Remark 3.9. We assume that $|\cdot|$ is non-trivial and $\|\cdot\|=\|\cdot\|_{\mathscr{H}}$ for some finitely generated lattice $\mathscr{H}$ of $H$. Then a free basis $\left(e_{1}, \ldots, e_{r}\right)$ of $\mathscr{H}$ yields an orthonormal basis of $H$ with respect to $\|\cdot\|$ (cf. Proposition 1.17). Moreover, $\mathscr{H}=(H,\|\cdot\|)_{\leq 1}$. 
3.3. Semipositive metric. We assume that $L$ is semiample, namely certain tensor power of $L$ is generated by global sections. We say that a continuous metric $h=\left\{|\cdot|_{h}(x)\right\}_{x \in X}$ an is semipositive if there are a sequence $\left\{e_{n}\right\}$ of positive integers and a sequence $\left\{\left(V_{n},\|\cdot\|_{n}\right)\right\}$ of normed finite-dimensional vector spaces over $k$ such that there is a surjective homomorphism $V_{n} \otimes_{k} \mathscr{O}_{X} \rightarrow L^{\otimes e_{n}}$ for every $n$, and that the sequence

$$
\left\{\frac{1}{e_{n}} \log \frac{|\cdot|_{\left(V_{n}, \mid \cdot \|_{n}\right)}^{\text {quot }}(x)}{|\cdot|_{h^{e_{n}}}(x)}\right\}_{n=1}^{\infty}
$$

converges to 0 uniformly on $X^{\text {an }}$.

Proposition 3.10. If $X$ is projective, $L$ is generated by global sections, and $h$ is semipositive, then the sequence

converges to 0 uniformly on $X^{\text {an }}$.

$$
\left\{\frac{1}{m} \log \frac{|\cdot|_{h^{m}}^{\text {quot }}(x)}{|\cdot|_{h^{m}}(x)}\right\}_{m=1}^{\infty}
$$

Proof. We set

$$
a_{m}=\max _{x \in X^{\mathrm{an}}}\left\{\log \frac{|\cdot|_{h^{m}}^{\text {quot }^{m}}(x)}{|\cdot|_{h^{m}}(x)}\right\} .
$$

Then $a_{m+m^{\prime}} \leq a_{m}+a_{m^{\prime}}$ by (3) in Lemma3.5, and hence $\lim _{m \rightarrow \infty} a_{m} / m=\inf \left\{a_{m} / m\right\}$ by Fekete's lemma. For $\epsilon>0$, there is $e_{n}$ such that

$$
e^{-e_{n} \epsilon}|\cdot|_{h^{e_{n}}}(x) \leq|\cdot|_{h_{n}}(x) \leq e^{e_{n} \epsilon}|\cdot|_{h^{e_{n}}}(x)
$$

for all $x \in X^{\text {an }}$, where $h_{n}=\left\{|\cdot|_{\left(V_{n},\|\cdot\|_{n}\right)}^{\text {quot }}(x)\right\}_{x \in X^{\text {an }}}$. Thus

$$
e^{-e_{n} \epsilon}\|\cdot\|_{h^{e_{n}}} \leq\|\cdot\|_{h_{n}} \leq e^{e_{n} \epsilon}\|\cdot\|_{h^{e_{n}}},
$$

so that $e^{-e_{n} \epsilon}|\cdot|_{h^{e_{n}}}^{\text {quot }}(x) \leq|\cdot|_{h_{n}}^{\text {quot }}(x) \leq e^{e_{n} \epsilon}|\cdot|_{h^{e_{n}}}^{\text {quot }}(x)$. Thus, by Proposition 3.6 ,

$$
e^{-e_{n} \epsilon}|\cdot|_{h^{2}}^{\text {quot }}(x) \leq|\cdot|_{h_{n}}(x) \leq e^{e_{n} \epsilon}|\cdot|_{h^{2}}^{\text {quot }}(x) \text {. }
$$

Therefore,

$$
1 \leq \frac{|\cdot|_{h^{e_{n}}}^{\text {quot }}(x)}{|\cdot|_{h^{e_{n}}}(x)}=\frac{|\cdot|_{h_{n}}(x)}{|\cdot|_{h^{e_{n}}}(x)} \frac{|\cdot|_{h^{e} n_{n}}^{\text {quot }}(x)}{|\cdot|_{h_{n}}(x)} \leq e^{2 e_{n} \epsilon},
$$

that is, $0 \leq a_{e_{n}} / e_{n} \leq 2 \epsilon$, and hence $0 \leq \lim _{m \rightarrow \infty} a_{m} / m \leq 2 \epsilon$, as required.

Corollary 3.11. A continuous metric $h$ is semipositive if and only if, for any $\epsilon>0$, there is a positive integer $n$ such that, for all $x \in X^{\text {an }}$, we can find $s \in H^{0}\left(X, L^{\otimes n}\right)_{\hat{\kappa}(x)} \backslash\{0\}$ with $\|s\|_{h^{n}, \hat{\kappa}(x)} \leq e^{n \epsilon}|s|_{h^{n}}(x)$.

Proof. First we assume that $h$ is semipositive. By using Proposition 3.10 , we can find a positive integer $n$ such that $L^{\otimes n}$ is generated by global sections and

$$
|\cdot|_{h^{n}}(x) \leq|\cdot|_{h^{n}}^{\text {quot }}(x) \leq e^{n \varepsilon / 2}|\cdot|_{h^{n}}(x)
$$

for all $x \in X^{\text {an }}$. On the other hand, there is $s \in H^{0}\left(X, L^{\otimes n}\right)_{\hat{\kappa}(x)} \backslash\{0\}$ such that $\|s\|_{h^{n}, \hat{k}(x)} \leq e^{n \epsilon / 2}|s|_{h^{n}}^{\text {quot }}(x)$. Thus,

$$
\|s\|_{h^{n}, \hat{\kappa}(x)} \leq e^{n \epsilon / 2}|s|_{h^{n}}^{\text {quot }}(x) \leq e^{n \epsilon}|s|_{h^{n}}(x) .
$$


Next we consider the converse. For a positive integer $m$, there is a positive integer $e_{m}$ such that, for any $x \in X^{\text {an }}$, we can find $s \in H^{0}\left(X, L^{\otimes e_{m}}\right)_{\hat{\kappa}(x)} \backslash\{0\}$ with $\|s\|_{h^{e m}, \hat{\kappa}(x)} \leq e^{e_{m} / m}|s|_{h^{e m}}(x)$. Clearly $L^{\otimes e_{m}}$ is generated by global sections. Moreover,

$$
|s|_{h^{e m}}(x) \leq|s|_{\left(H^{0}\left(X, L^{\otimes e m}\right),\|\cdot\|_{h} e^{m}\right)}^{\text {quot }}(x) \leq e^{e_{m} / m}|s|_{h^{e^{m}}}(x),
$$

that is,

$$
0 \leq \frac{1}{e_{m}} \log \left(\frac{|\cdot|_{\left(H^{0}\left(X, L^{\otimes e m}\right),\|\cdot\|_{h} e^{\text {quot }}\right.}^{\text {quot }}(x)}{|\cdot|_{h^{e m}}(x)}\right) \leq \frac{1}{m} .
$$

Thus $h$ is semipositive.

Corollary 3.12. Let $h$ be a continuous metric of $L^{\text {an }}$. If there are a sequence $\left\{e_{n}\right\}$ of positive integers and a sequence $\left\{h_{n}\right\}$ of metrics such that $h_{n}$ is a semipositive metric of $\left(L^{\otimes e_{n}}\right)^{\text {an }}$ for each $n$ and

$$
\frac{1}{e_{n}} \log \frac{|\cdot|_{h_{n}}(x)}{|\cdot|_{h^{e n}}(x)}
$$

converges to 0 uniformly as $n \rightarrow \infty$, then $h$ is semipositive.

Proof. For a positive number $\epsilon>0$, choose a positive integer $n$ such that

$$
e^{-\epsilon e_{n} / 3} h^{e_{n}} \leq h_{n} \leq e^{\epsilon e_{n} / 3} h^{e_{n}} .
$$

As $h_{n}$ is semipositive, by Corollary 3.11 there is a positive integer $m$ such that, for all $x \in X^{\text {an }}$, we can find $s \in H^{0}\left(X, L^{\otimes m e_{n}}\right)_{\hat{\kappa}(x)} \backslash\{0\}$ with $\|s\|_{h_{n}^{m}, \hat{\kappa}(x)} \leq e^{m e_{n} \epsilon / 3}|s|_{h_{n}^{m}}(x)$, so that

$$
\|s\|_{h^{m e_{n}, \hat{\kappa}(x)}} \leq e^{\epsilon m e_{n} / 3}\|s\|_{h_{n}^{m}, \hat{\kappa}(x)} \leq e^{2 m e_{n} \epsilon / 3}|s|_{h_{n}^{m}}(x) \leq e^{m e_{n} \epsilon}|s|_{h^{m e_{n}}}(x) .
$$

Therefore, the assertion follows from Corollary 3.11

3.4. The functions $\sigma$ and $\mu$ on $X^{\text {an }}$. Throughout this subsection, we assume that $X$ is projective. Let $\widehat{P i c}_{C^{0}}(X)$ denote the group of isomorphism classes of pairs $(L, h)$ consisting of an invertible sheaf $L$ on $X$ and a continuous metric $h$ of $L^{\text {an }}$. Fix $\bar{L}=(L, h) \in \widehat{\operatorname{Pic}}_{C^{0}}(X)$. We assume that $L$ is generated by global sections. We define $\sigma_{\bar{L}}(x)$ to be

$$
\sigma_{\bar{L}}(x):=\log \left(\frac{|\cdot|_{h}^{\text {quot }}(x)}{|\cdot|_{h}(x)}\right) .
$$

Lemma 3.13. For $\bar{L}$ and $\bar{L}^{\prime} \in \widehat{\operatorname{Pic}}_{C^{0}}(X)$ such that both $L$ and $L^{\prime}$ are generated by global sections, we have the following:

(1) $\sigma_{\bar{L}} \geq 0$ on $X^{\mathrm{an}}$.

(2) $\sigma_{\bar{L} \otimes \bar{L}^{\prime}}(x) \leq \sigma_{\bar{L}}(x)+\sigma_{\bar{L}^{\prime}}(x)$ for $x \in X^{\text {an }}$.

(3) If $\bar{L} \simeq \bar{L}^{\prime}$, then $\sigma_{\bar{L}}=\sigma_{\bar{L}^{\prime}}$ on $X^{\text {an }}$.

Proof. (1) and (3) are obvious. (2) follows from (3) in Lemma 3.5 .

We assume that $L$ is semiample. We set

$$
\mathbb{N}(L):=\left\{n \in \mathbb{Z}_{\geq 1} \mid L^{\otimes n} \text { is generated by global sections }\right\} .
$$


Note that $\mathbb{N}(L) \neq \varnothing$ and $\mathbb{N}(L)$ forms a subsemigroup of $\mathbb{Z}_{\geq 1}$ with respect to the addition of $\mathbb{Z}_{\geq 1}$. For $x \in X^{\text {an }}$, we define $\mu_{\bar{L}}(x)$ to be

$$
\mu_{\bar{L}}(x):=\inf \left\{\frac{\sigma_{\bar{L}^{\otimes n}}(x)}{n} \mid n \in \mathbb{N}(L)\right\} .
$$

Note that $\mu_{\bar{L}}$ is upper-semicontinuous on $X^{\text {an }}$ because $\sigma_{\bar{L}^{\otimes n}}$ is continuous for all $n \in \mathbb{N}(L)$. We set

$$
\widehat{\operatorname{Pic}}_{C^{0}}^{+}(X):=\left\{(L, h) \in \widehat{\operatorname{Pic}}_{C^{0}}(X) \mid L \text { is semiample }\right\} .
$$

Note that $\widehat{\operatorname{Pic}}_{C^{0}}^{+}(X)$ forms a semigroup with respect to $\otimes$.

Lemma 3.14. Let $\bar{L}=(L, h)$ and $\bar{L}^{\prime}=\left(L^{\prime}, h^{\prime}\right)$ be elements of $\widehat{\operatorname{Pic}}_{C^{0}}^{+}(X)$. Then we have the following:

(1) $\mu_{\bar{L}} \geq 0$ on $X^{\text {an }}$.

(2) $\mu_{\bar{L}}(x)=\lim _{\substack{n \rightarrow \infty \\ n \in \mathbb{N}(L)}} \frac{\sigma_{\bar{L}}^{\otimes n}(x)}{n}$ for $x \in X^{\text {an }}$.

(3) $\mu_{\bar{L} \otimes \bar{L}^{\prime}}(x) \leq \mu_{\bar{L}}(x)+\mu_{\bar{L}^{\prime}}(x)$ for $x \in X^{\text {an }}$.

(4) If $\bar{L} \simeq \bar{L}^{\prime}$, then $\mu_{\bar{L}}=\mu_{\bar{L}^{\prime}}$ on $X^{\text {an }}$.

(5) For $n \geq 0, \mu_{\bar{L}^{\otimes n}}=n \mu_{\bar{L}}$ on $X^{\text {an }}$.

Proof. (1) follows from (1) in Lemma 3.13 .

(2) Since $\sigma_{\bar{L}^{\otimes\left(n+n^{\prime}\right)}}(x) \leq \sigma_{\bar{L}^{\otimes n}}(x)+\sigma_{\bar{L}^{\otimes n^{\prime}}}(x)$ for $n, n^{\prime} \in \mathbb{N}(L)$ by (2) in Lemma3.13, the assertion follows from Fekete's lemma.

(3) and (4) follow from (2) and (3) in Lemma 3.13 together with (2), respectively.

(5) If $n=0$, then the assertion is obvious, so that we may assume that $n \geq 1$. We fix $n_{0} \in \mathbb{N}(L)$. Then $n_{0} \in \mathbb{N}\left(L^{\otimes n}\right)$. Thus, by (2),

$$
\mu_{\bar{L}^{\otimes n}}(x)=\lim _{m \rightarrow \infty} \frac{\sigma_{L^{\otimes m n} 0^{n}}(x)}{m n_{0}}=n \lim _{m \rightarrow \infty} \frac{\sigma_{L^{\otimes m n} 0^{n}}(x)}{m n_{0} n}=n \mu_{\bar{L}}(x) .
$$

We let $\widehat{\operatorname{Pic}}_{C^{0}}(X)_{\mathbb{Q}}$ be the quotient space of $\widehat{\operatorname{Pic}}_{C^{0}}(X) \otimes_{\mathbb{Z}} \mathbb{Q}$ by the Q-vector sub-

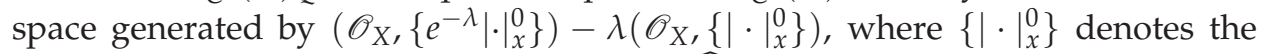
trivial continuous metric on $\mathscr{O}_{X}$. Note that $\widehat{\operatorname{Pic}}_{C^{0}}(X)_{\mathrm{Q}}$ can be identified with the Q-vector space of all pairs $(L, h)$, where $L$ is an element of $\widehat{\operatorname{Pic}}(X) \otimes \mathbb{Q}$ and $h$ is a continuous metric on $L$ (see \$1.1.5). Moreover, we set

$$
\widehat{\operatorname{Pic}}_{C^{0}}^{+}(X)_{\mathbb{Q}}:=\left\{(L, h) \in \widehat{\operatorname{Pic}}_{C^{0}}(X)_{\mathbb{Q}} \mid L \text { is semiample }\right\} .
$$

Let $\iota: \widehat{\operatorname{Pic}}_{C^{0}}(X) \rightarrow \widehat{\operatorname{Pic}}_{C^{0}}(X)_{\mathbb{Q}}$ be the canonical homomorphism. For $\bar{L} \in \widehat{\operatorname{Pic}}_{C^{0}}^{+}(X)_{Q^{\prime}}$, we choose a positive integer $n$ and $\bar{L}_{n} \in \widehat{\operatorname{Pic}}_{C^{0}}^{+}(X)$ with $\iota\left(\bar{L}_{n}\right)=\bar{L}^{\otimes n}$. Then $\mu_{\bar{L}_{n}}(x) / n$ does not depend on the choice of $n$ and $\bar{L}_{n}$. Indeed, let us choose another $n^{\prime} \in \mathbb{Z}_{\geq 1}$ and $\bar{L}_{n^{\prime}} \in \widehat{\operatorname{Pic}}_{C^{0}}^{+}(X)$ with $\iota\left(\bar{L}_{n^{\prime}}\right)=\bar{L}^{\otimes n^{\prime}}$. As $\iota\left(\bar{L}_{n}^{\otimes n^{\prime}}\right)=\iota\left(\bar{L}_{n^{\prime}}^{\otimes n}\right)=$ $\bar{L}^{\otimes n n^{\prime}}$, there is a positive integer $m$ such that $\bar{L}_{n}^{\otimes m n^{\prime}}=\bar{L}_{n^{\prime}}^{\otimes m n}$. By (5) in Lemma 3.14,

$$
m n^{\prime} \mu_{\bar{L}_{n}}(x)=\mu_{\bar{L}_{n}^{\otimes m n^{\prime}}}(x)=\mu_{\bar{L}_{n^{\prime}}^{\otimes m n}}(x)=m n \mu_{\bar{L}_{n^{\prime}}}(x),
$$


that is, $\mu_{\bar{L}_{n}}(x) / n=\mu_{\bar{L}_{n^{\prime}}}(x) / n^{\prime}$, as required. By abuse of notation, it is also denoted by $\mu_{\bar{L}}(x)$.

Lemma 3.15. For $\bar{L}, \bar{L}^{\prime} \in \widehat{\operatorname{Pic}}_{C^{0}}^{+}(X)_{Q^{Q}}$, we have the following:

(1) $\mu_{\bar{L} \otimes \bar{L}^{\prime}}(x) \leq \mu_{\bar{L}}(x)+\mu_{\bar{L}^{\prime}}(x)$ for $x \in X^{\text {an }}$.

(2) For $a \in \mathbb{Q}_{\geq 0}, \mu_{\bar{L}^{\otimes a}}=a \mu_{\bar{L}}$ on $X^{\mathrm{an}}$.

(3) Let $\bar{L}_{1}, \ldots, \bar{L}_{r}$ be elements of $\widehat{P i c}_{C_{0}}(X)_{\mathrm{Q}}$. We assume that there are open intervals $I_{1}, \ldots, I_{r}$ of $\mathbb{R}$ such that

$$
\bar{L} \otimes \bar{L}_{1}^{\otimes t_{1}} \otimes \cdots \otimes \bar{L}_{r}^{\otimes t_{r}} \in \widehat{\operatorname{Pic}}_{C^{0}}^{+}(X)_{\mathrm{Q}}
$$

for all $\left(t_{1}, \ldots, t_{r}\right) \in\left(I_{1} \times \cdots \times I_{r}\right) \cap \mathbb{Q}^{r}$. Then, for a fixed $x \in X^{\text {an }}$, there is a continuous function $f: I_{1} \times \cdots \times I_{r} \rightarrow \mathbb{R}$ such that

$$
f\left(t_{1}, \ldots, t_{r}\right)=\mu_{\bar{L} \otimes \bar{L}_{1}^{\otimes t_{1}} \otimes \cdots \otimes \bar{L}_{r}^{\otimes t_{r}}}(x)
$$

for all $\left(t_{1}, \ldots, t_{r}\right) \in\left(I_{1} \times \cdots \times I_{r}\right) \cap \mathbb{Q}^{r}$.

Proof. (1) and (2) are consequences of (3) and (5) in Lemma 3.14, respectively.

(3) We set

$$
f_{0}\left(t_{1}, \ldots, t_{r}\right):=\mu_{\bar{L} \otimes \bar{L}_{1}^{\otimes t_{1}} \otimes \cdots \otimes \bar{L}_{r}^{\otimes t_{r}}}(x)
$$

for $\left(t_{1}, \ldots, t_{r}\right) \in\left(I_{1} \times \cdots \times I_{r}\right) \cap \mathbb{Q}^{r}$. By (1) and (2), for $\lambda \in[0,1] \cap \mathbb{Q}$ and $\left(t_{1}, \ldots, t_{r}\right),\left(t_{1}^{\prime}, \ldots, t_{r}^{\prime}\right) \in\left(I_{1} \times \cdots \times I_{r}\right) \cap \mathbb{Q}^{r}$, we have

$$
\begin{aligned}
& f_{0}\left(\lambda\left(t_{1}, \ldots, t_{r}\right)+(1-\lambda)\left(t_{1}^{\prime}, \ldots, t_{r}^{\prime}\right)\right) \\
&= \mu_{\left(\bar{L} \otimes \bar{L}_{1}^{\otimes t_{1}} \otimes \cdots \otimes \bar{L}_{r}^{\otimes t_{r}}\right) \otimes \lambda \otimes\left(\bar{L} \otimes \bar{L}_{1}^{\otimes t_{1}^{\prime}} \otimes \cdots \otimes \bar{L}_{r}^{\otimes t_{r}^{\prime}}\right) \otimes(1-\lambda)}(x) \\
& \leq \lambda \mu_{\bar{L} \otimes \bar{L}_{1}^{\otimes t_{1}} \otimes \cdots \otimes \bar{L}_{r} t_{r}}(x)+(1-\lambda) \mu_{\bar{L} \otimes \bar{L}_{1}^{\otimes t_{1}^{\prime}} \otimes \cdots \otimes \bar{L}_{r}^{\otimes t_{r}^{\prime}}}(x) \\
& \quad=\lambda f_{0}\left(t_{1}, \ldots, t_{r}\right)+(1-\lambda) f_{0}\left(t_{1}^{\prime}, \ldots, t_{r}^{\prime}\right),
\end{aligned}
$$

that is, $f_{0}$ is concave on $\left(I_{1} \times \cdots \times I_{r}\right) \cap \mathbb{Q}^{r}$. Therefore, the assertion (3) follows from [13, Corollary 1.3.2].

Let $(L, h)$ be an element of $\widehat{\operatorname{Pic}}_{C^{0}}^{+}(X)_{\mathrm{Q}}$. We say that $h$ is semipositive if there is a positive integer $n$ such that $L^{\otimes n} \in \operatorname{Pic}(X)$ and $h^{n}$ is semipositive. The following characterization of the semipositivity of $h$ is a consequence of Proposition 3.10 .

Proposition 3.16. For $\bar{L}=(L, h) \in \widehat{\operatorname{Pic}}_{C^{0}}^{+}(X)_{Q}, h$ is semipositive if and only if $\mu_{\bar{L}}=0$ on $X^{\text {an }}$.

We assume that $|\cdot|$ is non-trivial. Let $\mathscr{X}$ be a model of $X$ over $\operatorname{Spec}\left(\mathfrak{o}_{k}\right)$. Let $L \in \operatorname{Pic}(X) \otimes \mathbb{Q}$ and $\mathscr{L} \in \operatorname{Pic}(\mathscr{X}) \otimes \mathbb{Q}$ with $\left.\mathscr{L}\right|_{X}=L$. Let $m$ be a positive integer such that $L^{\otimes m} \in \operatorname{Pic}(X)$. Then we define $\bar{L}=(L, h)$ to be

$$
(L, h):=\left(L^{\otimes m},\left\{|\cdot| \mathscr{L}^{\otimes m}(x)\right\}_{x \in X^{\text {an }}}\right)^{\otimes 1 / m} .
$$

Proposition 3.17. If $L$ is ample and $\mathscr{L}$ is nef, then $h$ is semipositive.

Proof. First we assume that $\mathscr{L}$ is ample. We choose a positive integer $n$ such that $\mathscr{L}^{\otimes n} \in \operatorname{Pic}(\mathscr{X})$ and $\mathscr{L}^{\otimes n}$ is very ample. Then we have an embedding $\iota$ : 
$\mathscr{X} \rightarrow \mathbb{P}\left(H^{0}\left(\mathscr{X}, \mathscr{L}^{\otimes n}\right)\right)$ and $\mathscr{L}^{\otimes n}=\iota^{*}\left(\mathscr{O}_{\mathbb{P}\left(H^{0}\left(\mathscr{X}, \mathscr{L}_{\otimes n}\right)\right)}(1)\right)$. Let $\left(e_{1}, \ldots, e_{r}\right)$ be a free basis of $H^{0}\left(\mathscr{X}, \mathscr{L}^{\otimes n}\right)$. We define a norm $\|\cdot\|$ of $H^{0}\left(X, L^{\otimes n}\right)$ to be

$$
\left\|a_{1} e_{1}+\cdots+a_{r} e_{r}\right\|:=\max \left\{\left|a_{1}\right|, \ldots,\left|a_{r}\right|\right\} .
$$

Note that $\left(H^{0}\left(X, L^{\otimes n}\right),\|\cdot\|\right)_{\leq 1}=H^{0}\left(\mathscr{X}, \mathscr{L}^{\otimes n}\right)$, so that, by Proposition 3.8 we have $|\cdot|_{(H,\|\cdot\|)}^{\text {quot }}(x)=|\cdot|_{\mathscr{L} \otimes n}(x)$ for $x \in X^{\text {an }}$. Thus $h$ is semipositive.

In general, let $\mathscr{A}$ be an ample invertible sheaf on $\mathscr{X}$ and $A:=\left.\mathscr{A}\right|_{\mathrm{X}}$. We choose $\delta \in \mathbb{Q}_{>0}$ such that $L \otimes A^{\otimes a}$ is ample for all $a \in(-\delta, \delta) \cap \mathbb{Q}$. Note that

$$
\bar{L} \otimes(A,|\cdot| \mathscr{A})^{\otimes \epsilon}=\left(L \otimes A^{\otimes \epsilon},|\cdot| \mathscr{L} \otimes \mathscr{A} \otimes \epsilon\right),
$$

so that $\mu_{\bar{L} \otimes(A,|\cdot| \mathscr{A})^{\otimes \epsilon}}=0$ for $\epsilon \in(0, \delta) \cap \mathbb{Q}$ by the previous observation together with Proposition 3.16. On the other hand, by (3) in Lemma 3.15.

$$
\mu_{\bar{L}}(x)=\lim _{\substack{\epsilon \downarrow 0 \\ \epsilon \in \mathbb{Q}}} \mu_{\bar{L} \otimes(A,|\cdot| \mathscr{A})^{\otimes \epsilon}}(x) .
$$

Therefore, $\mu_{\bar{L}}=0$, and hence $h$ is semipositive by Proposition 3.16

Remark 3.18. Assume that the absolute value $|\cdot|$ is non-trivial. Let $L$ be an ample invertible sheaf on $X$, equipped with a semipositive continuous metric $h$. Then there exists a sequence $\left\{\left(\mathscr{X}_{n}, \mathscr{L}_{n}\right)\right\}_{n} \geqslant 1$, where $\mathscr{X}_{n}$ is a model of $X$ and $\mathscr{L}_{n}$ is a nef invertible sheaf on $\mathscr{X}_{n}$ such that $\left.\mathscr{L}_{n}\right|_{X}=L^{\otimes n}$ and that $h_{n}=\left(|\cdot|_{\mathscr{L}_{n}}(x)^{1 / n}\right)_{x \in X^{\text {an }}}$ converges uniformly to $h$. This follows from Proposition 3.10 and the comparison between quotient metrics and model metrics (via the embedding into the projective spaces of lattices). Combining with Proposition 3.17 and Corollary 3.11 we obtain that, in the non-trivial valuation case, our semipositivity coincides with that of Zhang [20] and Moriwaki [15]. We refer the readers to [11, §6] and to [7. §6.8] for the descriptions of the semipositivity in terms of plurisubharmonic currents. Note that their semipositivity is also equivalent to our semipositivity.

\section{EXTENSION THEOREM}

Throughout this section, we assume that $X$ is projective. Let us begin with a special case of the extension theorem. The general extension theorem is a consequence of the special case.

Theorem 4.1. We assume that $L$ is very ample. Let $\|\cdot\|$ be a norm of $H^{0}(X, L)$ and $h$ a continuous metric of $L^{\text {an }}$ given by $\left\{|\cdot|_{\left(H^{0}(X, L),\|\cdot\|\right)}^{\text {quot }}(x)\right\}_{x \in X^{\text {an }}}$. Let $Y$ be a closed subscheme of $X$ and $l \in H^{0}\left(Y,\left.L\right|_{Y}\right)$. Then, for any $\epsilon>0$, there are a positive integer $n$ and $s \in H^{0}\left(X, L^{\otimes n}\right)$ such that $\left.s\right|_{Y}=l^{\otimes n}$ and $\|s\|_{h^{\otimes n}} \leq e^{n \epsilon}\left(\|l\|_{Y, h}\right)^{n}$.

Proof. First we assume that $|\cdot|$ is non-trivial. Let us begin with the following:

Claim 4.1.1. There are a positive integer a and a finitely generated lattice $\mathscr{H}$ of $H^{0}\left(X, L^{\otimes a}\right)$ such that

$$
\|\cdot\|_{h^{a}} \leq\|\cdot\|_{\mathscr{H}} \leq e^{a \epsilon / 2}\|\cdot\|_{h^{a}}
$$

Proof. First we assume that $|\cdot|$ is discrete. We choose a positive integer $a$ such that $|\omega|^{-1} \leq e^{a \epsilon / 2}$. We set $\mathscr{H}:=\left\{s \in H^{0}\left(X, L^{\otimes a}\right) \mid\|s\|_{h^{a}} \leq 1\right\}$. Note that $\mathscr{H}$ is a finitely generated lattice of $H^{0}\left(X, L^{\otimes a}\right)$ by Proposition 1.20, As $\|\cdot\|_{h^{a}} \leq\|\cdot\|_{\mathscr{H}} \leq$ $|\omega|^{-1}\|\cdot\|_{h^{a}}$ by Proposition 1.20 we have the assertion. 
Next we assume that $|\cdot|$ is not discrete. By Proposition 1.21, there is a lattice $\mathscr{V}$ of $H^{0}(X, L)$ such that $\|\cdot\|_{h}=\|\cdot\|_{\mathscr{V}}$. By Proposition 1.22, there is a finitely generated lattice $\mathscr{H}$ of $H^{0}(X, L)$ such that $\mathscr{H} \subseteq \mathscr{V}$ and $\|\cdot\|_{h} \leq\|\cdot\|_{\mathscr{H}} \leq e^{\epsilon / 2}\|\cdot\|_{h}$, as desired.

Let $\mathscr{X}$ be the Zariski closure of $X$ in $\mathbb{P}(\mathscr{H})$ (cf. \$1.1.7) and $\mathscr{L}=\left.\mathscr{O}_{\mathbb{P}(\mathscr{H})}(1)\right|_{\mathscr{X}}$. Moreover, let $h^{\prime}$ be a continuous metric of $\left(L^{\otimes a}\right)^{\text {an }}$ given by

$$
\left\{|\cdot|_{\left(H,\|\cdot\|_{\mathscr{H}}\right)}^{\text {quot }}(x)\right\}_{x \in X^{\text {an }}} \text {. }
$$

Then, by Proposition 3.8 and Remark 3.9. $|\cdot|_{h^{\prime}}=|\cdot|_{\mathscr{L}}$. Therefore, by virtue of Theorem 3.2 there are a positive integer $m$ and $s \in H^{0}\left(X, L^{\otimes a m}\right)$ such that $\left.s\right|_{Y}=$ $l^{\otimes a m}$ and

$$
\|s\|_{h^{\prime m}} \leq e^{a m \epsilon / 2}\left(\left\|l^{\otimes a}\right\|_{Y, h^{\prime}}\right)^{m} .
$$

As $\|\cdot\|_{h^{a}} \leq\|\cdot\|_{\mathscr{H}} \leq e^{a \epsilon / 2}\|\cdot\|_{h^{a}}$, we have

$$
|\cdot|_{h^{a}}^{\text {quot }}(x) \leq|\cdot|_{h^{\prime}}(x) \leq e^{a \epsilon / 2}|\cdot|_{h^{a}}^{\text {quot }}(x)
$$

for all $x \in X^{\text {an }}$. Therefore, by Proposition 3.6 .

$$
|\cdot|_{h^{a}}(x) \leq|\cdot|_{h^{\prime}}(x) \leq e^{a \epsilon / 2}|\cdot|_{h^{a}}(x)
$$

for all $x \in X^{\text {an }}$. In particular, $|\cdot|_{h^{a m}}(x) \leq|\cdot|_{h^{\prime m}}(x)$. Therefore,

$$
\|s\|_{h^{a m}} \leq\|s\|_{h^{\prime m}}
$$

On the other hand, by using (4.2),

$$
\left\|l^{\otimes a}\right\|_{Y, h^{\prime}} \leq e^{a \epsilon / 2} \sup \left\{\left|l^{\otimes a}\right|_{h^{a}}(y) \mid y \in Y^{\mathrm{an}}\right\} \leq e^{a \epsilon / 2}\left(\|l\|_{Y, h}\right)^{a} .
$$

Thus the assertion follows from (4.1), 4.3) and (4.4).

Next we assume that $|\cdot|$ is trivial. Clearly we may assume that $l \neq 0$. Let $k^{\prime}$ be the field $k((T))$ of formal Laurent power series over $k$, that is, the quotient field of the ring $k \llbracket T \rrbracket$ of formal power series over $k$. We set

$$
\Sigma:=\bigcup_{i=0}^{\infty}\left(\bigcup_{s, s^{\prime} \in H^{0}\left(X, L^{\otimes i}\right) \backslash\{0\}} \mathbb{Q}\left(\log \|s\|_{h^{i}}-\log \left\|s^{\prime}\right\|_{h^{i}}\right)\right) .
$$

As $\left\{\|s\|_{h^{i}} \mid s \in H^{0}\left(X, L^{\otimes i}\right) \backslash\{0\}\right\}$ is a finite set by (1) in Lemma 1.15, we have $\#(\Sigma) \leq \aleph_{0}$. Therefore, we can find $\alpha \in \mathbb{R}_{>0} \backslash \Sigma$. Here we consider an absolute value $|\cdot|^{\prime}$ of $k^{\prime}$ given by

$$
|\phi(T)|^{\prime}:=\exp (-\alpha \operatorname{ord}(\phi(T))) \quad\left(\phi(T) \in k^{\prime}\right) .
$$

We set

$$
X^{\prime}:=X \times_{\operatorname{Spec}(k)} \operatorname{Spec}\left(k^{\prime}\right), \quad Y^{\prime}:=Y \times_{\operatorname{Spec}(k)} \operatorname{Spec}\left(k^{\prime}\right) \quad \text { and } \quad L^{\prime}=L \otimes_{k} k^{\prime} .
$$

Note that $H^{0}\left(X^{\prime}, L^{\prime}\right)=H^{0}(X, L) \otimes_{k} k^{\prime}$. Let $h^{\prime}$ be a continuous metric of $L^{\prime \text { an }}$ given by the scalar extension of $h$. Then, by Lemma $3.7 h^{\prime}$ is given by

$$
\left\{|\cdot|_{\left(H^{0}\left(X^{\prime}, L^{\prime}\right),\|\cdot\|_{k^{\prime}}\right)}^{\text {quot }}\left(x^{\prime}\right)\right\}_{x^{\prime} \in X^{\prime a n},}
$$


where $\|\cdot\|_{k^{\prime}}$ is the scalar extension of $\|\cdot\|$. Moreover, for $s \in H^{0}(X, L),|s|_{h^{\prime}}\left(x^{\prime}\right)=$ $|s|_{h}\left(p^{\text {an }}\left(x^{\prime}\right)\right)$ for $x^{\prime} \in X^{\prime a n}$, where $p: X^{\prime} \rightarrow X$ is the projection. Note that $p^{\text {an }}:$ $X^{\prime a n} \rightarrow X^{\text {an }}$ is surjective. Therefore, $\|s\|_{h^{\prime}}=\|s\|_{h}$ for all $s \in H^{0}(X, L)$.

By the previous observation, there are a positive integer $n$ and $s^{\prime} \in H^{0}\left(X^{\prime}, L^{\prime \otimes n}\right)$ such that

$$
\left.s^{\prime}\right|_{Y^{\prime}}=l^{\otimes n} \quad \text { and } \quad\left\|s^{\prime}\right\|_{h^{\prime n}} \leq e^{n \epsilon}\left(\|l\|_{Y^{\prime}, h^{\prime}}\right)^{n}=e^{n \epsilon}\left(\|l\|_{Y, h}\right)^{n} .
$$

Note that, for a positive integer $d$,

$$
s^{\prime \otimes d} \in H^{0}\left(X^{\prime}, L^{\prime \otimes d n}\right),\left.\quad s^{\otimes d}\right|_{Y^{\prime}}=l^{\otimes d n} \quad \text { and } \quad\left\|s^{\otimes d}\right\|_{h^{\prime d n}} \leq e^{d n \epsilon}\left(\|l\|_{Y, h}\right)^{d n} .
$$

Thus we may assume that $H^{0}\left(X, L^{\otimes n}\right) \rightarrow H^{0}\left(Y,\left.L\right|_{Y} ^{\otimes n}\right)$ is surjective. Let $\left(e_{1}, \ldots, e_{r}\right)$ be an orthogonal basis of $H^{0}\left(X, L^{\otimes n}\right)$ with respect to $\|\cdot\|_{h^{n}}$ such that $\left(e_{t+1}, \ldots, e_{r}\right)$ forms a basis of $\operatorname{Ker}\left(H^{0}\left(X, L^{\otimes n}\right) \rightarrow H^{0}\left(Y,\left.L\right|_{Y} ^{\otimes n}\right)\right)$ (cf. Proposition 1.3). We set

$$
s^{\prime}=a_{1}(T) e_{1}+\cdots+a_{t}(T) e_{t}+a_{t+1}(T) e_{t+1}+\cdots+a_{r}(T) e_{r}
$$

for some $a_{1}(T), \ldots, a_{r}(T) \in k^{\prime}=k((T))$. As $\left.s^{\prime}\right|_{Y^{\prime}}=l^{\otimes n} \in H^{0}\left(Y,\left.L\right|_{Y} ^{\otimes n}\right)$ and $\left(\left.e_{1}\right|_{Y}, \ldots,\left.e_{t}\right|_{Y}\right)$ forms a basis of $H^{0}\left(Y,\left.L\right|_{Y} ^{\otimes n}\right)$, we have $a_{1}(T), \ldots, a_{t}(T) \in k$. Note that

$$
\alpha \notin \bigcup_{s, s^{\prime} \in H^{0}\left(X, L^{\otimes n}\right) \backslash\{0\}} \mathbb{Q}\left(\log \|s\|_{h^{n}}-\log \left\|s^{\prime}\right\|_{h^{n}}\right),
$$

so that, by (2) in Lemma 1.15 and Remark 1.16, $\left(e_{1}, \ldots, e_{r}\right)$ forms an orthogonal basis of $H^{0}\left(X^{\prime}, L^{\prime \otimes n}\right)$ with respect to $\|\cdot\|_{h^{\prime \prime}}$. Therefore, if we set $s=a_{1} e_{1}+\cdots+$ $a_{t} e_{t}$, then $s \in H^{0}\left(X, L^{\otimes n}\right),\left.s\right|_{Y}=l^{\otimes n}$ and

$$
\begin{aligned}
\|s\|_{h^{n}} & =\max \left\{\left|a_{1}\right|\left\|e_{1}\right\|_{h^{n}}, \ldots,\left|a_{t}\right|\left\|e_{t}\right\|_{h^{n}}\right\} \\
& \leq \max \left\{\left|a_{1}\right|\left\|e_{1}\right\|_{h^{n}}, \ldots,\left|a_{t}\right|\left\|e_{t}\right\|_{h^{n}},\left|a_{t+1}(T)\right|^{\prime}\left\|e_{t+1}\right\|_{h^{n}}, \ldots,\left|a_{r}(T)\right|^{\prime}\left\|e_{r}\right\|_{h^{n}}\right\} \\
& =\left\|s^{\prime}\right\|_{h^{\prime n}} \leq e^{n \epsilon}\left(\|l\|_{Y, h}\right)^{n},
\end{aligned}
$$

as required.

Theorem 4.2. We assume that $L$ is ample and $h$ is a semipositive continuous metric of $L^{\text {an }}$. Fix a closed subscheme $Y, l \in H^{0}\left(Y,\left.L\right|_{Y}\right)$ and $\in \in \mathbb{R}_{>0}$. Then there is a positive integer $n_{0}$ such that, for all $n \geq n_{0}$, we can find $s \in H^{0}\left(X, L^{\otimes n}\right)$ with

$$
\left.s\right|_{Y}=l^{\otimes n} \text { and }\|s\|_{h^{n}} \leq e^{n \epsilon}\left(\|l\|_{Y, h}\right)^{n} .
$$

Proof. Clearly we may assume that $l \neq 0$. Let us begin with the following claim:

Claim 4.2.1. For any $\epsilon^{\prime}>0$, there are a positive integer $N$ and $s_{N} \in H^{0}\left(X, L^{\otimes N}\right)$ such that

$$
\left.s_{N}\right|_{Y}=l^{\otimes N} \text { and }\left\|s_{N}\right\|_{h^{N}} \leq e^{N \epsilon^{\prime}}\left(\|l\|_{Y, h}\right)^{N} .
$$

Proof. By using Proposition 3.10, we can find a positive integer $a$ such that $L^{\otimes a}$ is very ample and

$$
|\cdot|_{h^{a}}(x) \leq|\cdot|_{h^{a}}^{\text {quot }}(x) \leq e^{a \epsilon^{\prime} / 2}|\cdot|_{h^{a}}(x)
$$

for all $x \in X^{\text {an }}$. We set $h^{\prime}=\left\{|\cdot|_{h^{a}}^{\text {quot }}(x)\right\}$. Then, the above inequalities means that

$$
|\cdot|_{h^{a}}(x) \leq|\cdot|_{h^{\prime}}(x) \leq e^{a \epsilon^{\prime} / 2}|\cdot|_{h^{a}}(x)
$$


for all $x \in X^{\text {an }}$. Further, by Theorem 4.1 there are a positive integer $b$ and $s_{a b} \in H^{0}\left(X, L^{\otimes a b}\right)$ such that $\left.s_{a b}\right|_{Y}=l^{\otimes a b}$ and

$$
\left\|s_{a b}\right\|_{h^{\prime b}} \leq e^{a b \epsilon^{\prime} / 2}\left(\left\|l^{\otimes a}\right\|_{Y, h^{\prime}}\right)^{b} .
$$

By (4.5),

$$
\left\|l^{\otimes a}\right\|_{Y, h^{\prime}} \leq e^{a \epsilon^{\prime} / 2}\left\|l^{\otimes a}\right\|_{Y, h^{a}}=e^{a \epsilon^{\prime} / 2}\left(\|l\|_{Y, h}\right)^{a} .
$$

Moreover, as $|\cdot|_{h^{a b}}(x) \leq|\cdot|_{h^{\prime b}}(x)$ by (4.5), we have $\left\|s_{a b}\right\|_{h^{a b}} \leq\left\|s_{a b}\right\|_{h^{\prime b}}$, so that

$$
\begin{aligned}
\left\|s_{a b}\right\|_{h^{a b}} & \leq\left\|s_{a b}\right\|_{h^{\prime b}} \leq e^{a b \epsilon^{\prime} / 2}\left(\left\|l^{\otimes a}\right\|_{Y, h^{\prime}}\right)^{b} \\
& \leq e^{a b \epsilon^{\prime} / 2}\left(e^{a \epsilon^{\prime} / 2}\left(\|l\|_{Y, h}\right)^{a}\right)^{b} \leq e^{a b \epsilon^{\prime}}\left(\|l\|_{Y, h}\right)^{a b} .
\end{aligned}
$$

Therefore, if we set $N=a b$, then we have the assertion of the claim.

Since $L$ is ample, by Corollary 1.2 , the above claim is actually equivalent to the assertion of the theorem. Thus the theorem is proved.

\section{Arithmetic NaKai-Moishezon Criterion Over a NUmber Field}

In this section, as an application of the extension property (cf. [16] and Theorem 4.2), we consider the arithmetic Nakai-Moishezon criterion over a number field under a weaker assumption (adelically normed vector space) than Zhang's paper [20].

5.1. Adelically normed vector space over a number field. Fix a number field $K$. Let $\mathcal{O}_{K}$ be the ring of integers in $K$. We set

$$
\left\{\begin{array}{l}
M_{K}^{\mathrm{fin}}:=\operatorname{Spec}\left(\mathcal{O}_{K}\right) \backslash\{(0)\}, \\
M_{K}^{\infty}:=K(\mathbb{C})(=\text { the set of all embeddings } K \hookrightarrow \mathbb{C}) .
\end{array}\right.
$$

Moreover, $M_{K}:=M_{K}^{\text {fin }} \cup M_{K}^{\infty}$. For $\mathfrak{p} \in M_{K}^{\text {fin }}$ and $\sigma \in M_{K}^{\infty}$, the absolute values $|\cdot|_{\mathfrak{p}}$ and $|\cdot|_{\sigma}$ of $K$ are defined by

$$
|x|_{\mathfrak{p}}:=\#\left(\mathcal{O}_{K} / \mathfrak{p}\right)^{-\operatorname{ord}_{\mathfrak{p}}(x)} \text { and }|x|_{\sigma}:=|\sigma(x)| \quad(x \in K),
$$

respectively. Further, for $\mathfrak{p} \in M_{K}^{\text {fin }}$, the completion of $K$ with respect to $|\cdot|_{\mathfrak{p}}$ is denoted by $K_{\mathfrak{p}}$. In addition, $K_{\sigma}$ and $K \hookrightarrow K_{\sigma}\left(\sigma \in M_{K}^{\infty}\right)$ are defined to be $\mathbb{C}$ and $\sigma$, respectively. By abuse of notation, for $v \in M_{K}$, the extension absolute of $|\cdot|_{v}$ to $K_{v}$ is also denoted by $|\cdot|_{v}$. In the case where $v=\sigma \in M_{K}^{\infty},|\cdot|_{\sigma}$ on $K_{\sigma}=\mathbb{C}$ is the usual absolute value. If $\mathfrak{p} \in M_{K}^{\text {fin }}$, the valuation rings of $\left(K,|\cdot|_{\mathfrak{p}}\right)$ and $\left(K_{\mathfrak{p}},|\cdot|_{\mathfrak{p}}\right)$ are denoted by $\mathcal{O}_{\mathfrak{p}}$ and $\widehat{\mathcal{O}}_{\mathfrak{p}}$, respectively. Note that $\mathcal{O}_{\mathfrak{p}}$ is the localization of $\mathcal{O}_{K}$ with respect to $\mathcal{O}_{K} \backslash \mathfrak{p}$, and $\widehat{\mathcal{O}}_{\mathfrak{p}}$ is the completion of the local ring $\mathcal{O}_{\mathfrak{p}}$.

Definition 5.1. Let $H$ be a finite-dimensional vector space over $K$. For $v \in M_{K}$, $H \otimes_{K} K_{v}$ is denoted by $H_{v}$. For each $v \in M_{K}$, let $\|\cdot\|_{v}$ be a norm of $H_{v}$ over $\left(K_{v},|\cdot|_{v}\right)$. In the case where $v \in M_{K}^{\text {fin }}$, the norm $\|\cdot\|_{v}$ is always assumed to be ultrametric. Moreover, we assume that the family $\left(\|\cdot\|_{\sigma}\right)_{\sigma \in M_{K}^{\infty}}$ is invariant under the complex conjugation, namely for any finite family of vectors $\left(s_{i}\right)_{i=1}^{n}$ in $H$ and vector $\left(\lambda_{i}\right)_{i=1}^{n}$ of complex numbers, one has

$$
\left\|\overline{\lambda_{1}} \otimes s_{1}+\cdots+\overline{\lambda_{n}} \otimes s_{n}\right\|_{\bar{\sigma}}=\left\|\lambda_{1} \otimes s_{1}+\cdots+\lambda_{n} \otimes s_{n}\right\|_{\sigma} .
$$


The family $\left\{\|\cdot\|_{v}\right\}_{v \in M_{K}}$ of norms is often denoted by $\|\cdot\|$. The pair $(H,\|\cdot\|)$ is called an adelically normed vector space over $K$ if, for any $x \in H,\|x\|_{\mathfrak{p}} \leq 1$ except finitely many $\mathfrak{p} \in M_{K}^{\text {fin }}$ (cf. [5, Definition 2.1] and [6, Definition 2.10]). We set

$$
\left\{\begin{array}{l}
(H,\|\cdot\|)_{\leq 1}^{\text {fin }}:=\left\{x \in H \mid\|x\|_{\mathfrak{p}} \leq 1 \text { for all } \mathfrak{p} \in M_{K}^{\mathrm{fin}}\right\}, \\
(H,\|\cdot\|)_{\leq 1}^{\mathfrak{p}}:=\left\{x \in H \mid\|x\|_{\mathfrak{p}} \leq 1\right\} .
\end{array}\right.
$$

Lemma 5.2. We assume that $(H,\|\cdot\|)$ is an adelically normed vector space over $K$.

(1) For $\mathfrak{p} \in M_{K}^{\mathrm{fin}},(H,\|\cdot\|)_{\leq 1}^{\mathfrak{p}}=(H,\|\cdot\|)_{\leq 1}^{\mathrm{fin}} \otimes_{\mathcal{O}_{K}} \mathcal{O}_{\mathfrak{p}}$.

(2) $(H,\|\cdot\|)_{\leq 1}^{\text {fin }}$ is a finitely generated $\mathcal{O}_{K}$-module and $(H,\|\cdot\|)_{\leq 1}^{\text {fin }} \otimes_{\mathcal{O}_{K}} K=H$. Moreover, $(H,\|\cdot\|)_{<1}^{\text {fin }} \otimes_{\mathbb{Z}} \mathbb{Q}=H$.

(3) Let $f: H \rightarrow H^{\prime}$ be a surjective homomorphism of finite-dimensional vector spaces over $K$. Let $\|\cdot\|_{v}^{\text {quot }}$ be the quotient norm of $H_{v}^{\prime}$ induced by the surjection $f_{v}: H_{v} \rightarrow H_{v}^{\prime}$ and the norm $\|\cdot\|_{v}$ on $H_{v}$. Then $\left(H^{\prime},\|\cdot\|^{\text {quot }}\right)$ is an adelically normed vector space over $\mathrm{K}$ and

$$
\begin{aligned}
& f\left((H,\|\cdot\|)_{\leq 1}^{\text {fin }}\right)=\left(H^{\prime},\|\cdot\|^{\text {quot }}\right)_{\leq 1}^{\text {fin }}, \\
& \text { where }\|\cdot\|{ }^{\text {quot }}=\left\{\|\cdot\|_{v}^{\text {quot }}\right\}_{v \in M_{K}} .
\end{aligned}
$$

Proof. (1) Obviously $(H,\|\cdot\|)_{\leq 1}^{\text {fin }} \otimes_{\mathcal{O}_{K}} \mathcal{O}_{\mathfrak{p}} \subseteq(H,\|\cdot\|)_{\leq 1}^{\mathfrak{p}}$. Conversely, we assume that $x \in H$ and $\|x\|_{\mathfrak{p}} \leq 1$. We set

$$
\left\{\mathfrak{q} \in M_{K}^{\text {fin }} \mid\|x\|_{\mathfrak{q}}>1\right\}=\left\{\mathfrak{q}_{1}, \ldots, \mathfrak{q}_{r}\right\} .
$$

By Lemma 5.3 as below, there is $\alpha \in K^{\times}$such that

$$
\operatorname{ord}_{\mathfrak{q}_{i}}(\alpha)>0(\forall i=1, \ldots, r) \text { and } \operatorname{ord}_{\mathfrak{q}}(\alpha)=0\left(\forall \mathfrak{q} \in M_{K}^{\text {fin }} \backslash\left\{\mathfrak{q}_{1}, \ldots, \mathfrak{q}_{r}\right\}\right) \text {. }
$$

We choose a positive integer $n$ such that $\left\|\alpha^{n} x\right\|_{\mathfrak{q}_{i}} \leq 1$ for all $i=1, \ldots, r$. Note that $\alpha^{n} \in \mathcal{O}_{\mathfrak{p}}^{\times}$and $\alpha^{n} x \in(H,\|\cdot\|)_{\leq 1}^{\text {fin }}$, so that $x=\alpha^{-n} \alpha^{n} x \in(H,\|\cdot\|)_{\leq 1}^{\text {fin }} \otimes_{\mathcal{O}_{K}} \mathcal{O}_{\mathfrak{p}}$.

(2) Since $(H,\|\cdot\|)_{\leq 1}^{\mathfrak{p}}$ is a finitely generated $\mathcal{O}_{\mathfrak{p}}$-module by Proposition 1.6, (1) implies that $(H,\|\cdot\|)_{\leq 1}^{\text {fin }} \otimes_{\mathcal{O}_{K}} \mathcal{O}_{\mathfrak{p}}$ is finitely generated for all $\mathfrak{p} \in M_{K}^{\text {fin }}$. Thus the first assertion follows.

For $x \in H$, by using Lemma 5.3, we can find $\beta \in \mathcal{O}_{K} \backslash\{0\}$ with $\beta x \in$ $(H,\|\cdot\|)_{\leq 1}$, which means that the second assertion holds.

Let $\gamma \in \mathcal{O}_{K} \backslash\{0\}$. Then there are $a_{1}, \ldots, a_{n} \in \mathbb{Z}$ such that

$$
\gamma^{n}+a_{1} \gamma^{n-1}+\cdots+a_{n}=0 .
$$

Clearly we may assume that $a_{n} \neq 0$. Thus, if we set

$$
\gamma^{\prime}=-\left(\gamma^{n-1}+a_{1} \gamma^{n-1}+\cdots+a_{n-1}\right),
$$

then $\gamma^{\prime} \in \mathcal{O}_{K}$ and $\gamma \gamma^{\prime}=a_{n}$. Note that $(H,\|\cdot\|)_{\leq 1}^{\text {fin }} \otimes_{\mathcal{O}_{K}} K$ and $(H,\|\cdot\|)_{\leq 1}^{\text {fin }} \otimes_{\mathbb{Z}} \mathbb{Q}$ are the localizations of $(H,\|\cdot\|)_{\leq 1}^{\text {fin }}$ with respect to $\mathcal{O}_{K} \backslash\{0\}$ and $\mathbb{Z} \backslash\{0\}$, respectively. Therefore the last assertion follows.

(3) The first assertion is obvious. Let us see that

$$
f\left((H,\|\cdot\|)_{\leq 1}^{\mathfrak{p}}\right)=\left(H^{\prime},\|\cdot\|^{\text {quot }}\right)_{\leq 1}^{\mathfrak{p}}
$$


for all $\mathfrak{p} \in M_{K}^{\text {fin }}$. Clearly one has $f\left((H,\|\cdot\|)_{\leq 1}^{\mathfrak{p}}\right) \subseteq\left(H^{\prime},\|\cdot\|^{\text {quot }}\right)_{\leq 1}^{\mathfrak{p}}$. The converse inclusion follows from Proposition 1.7. By using (1) together with the equation (5.1), we obtain

$$
f\left((H,\|\cdot\|)_{\leq 1}^{\text {fin }}\right) \otimes_{\mathcal{O}_{K}} \mathcal{O}_{\mathfrak{p}}=\left(H^{\prime},\|\cdot\|^{\text {quot }}\right)_{\leq 1}^{\text {fin }} \otimes_{\mathcal{O}_{K}} \mathcal{O}_{\mathfrak{p}} .
$$

Therefore (3) follows from [1, Proposition 3.8].

Lemma 5.3. Let $\Sigma$ be a finite subset of $M_{K}^{\mathrm{fin}}$. Then there is $\alpha \in K^{\times}$such that

$$
\operatorname{ord}_{\mathfrak{p}}(\alpha) \begin{cases}>0 & \text { if } \mathfrak{p} \in \Sigma \\ =0 & \text { if } \mathfrak{p} \in M_{K}^{\text {fin }} \backslash \Sigma .\end{cases}
$$

Proof. We set $\Sigma=\left\{\mathfrak{p}_{1}, \ldots, \mathfrak{p}_{e}\right\}$. As the class group of $K$ is finite, for each $i$, there are a positive integer $n_{i}$ and $\alpha_{i} \in \mathcal{O}_{K} \backslash\{0\}$ with $\mathfrak{p}_{i}^{n_{i}}=\alpha_{i} \mathcal{O}_{K}$. Thus, if we set $\alpha=\alpha_{1} \cdots \alpha_{e}$, then the assertion follows.

5.2. Estimation of $\lambda_{\mathrm{Q}}$ for a graded algebra. A normed $\mathbb{Z}$-module is a pair $(\mathscr{M},\|\cdot\|)$ of a finitely generated $\mathbb{Z}$-module $\mathscr{M}$ and a norm $\|\cdot\|$ of $\mathscr{M} \otimes_{\mathbb{Z}} \mathbb{R}$. We define $\lambda_{\mathbb{Q}}(\mathscr{M},\|\cdot\|)$ and $\lambda_{\mathbb{Z}}(\mathscr{M},\|\cdot\|)$ as follows. If $\mathscr{M}$ is a torsion module, then

$$
\lambda_{\mathrm{Q}}(\mathscr{M},\|\cdot\|)=\lambda_{\mathbb{Z}}(\mathscr{M},\|\cdot\|)=0 .
$$

Otherwise, let $\lambda_{\mathbb{Q}}(\mathscr{M},\|\cdot\|)$ (resp. $\left.\lambda_{\mathbb{Z}}(\mathscr{M},\|\cdot\|)\right)$ be the infimum of the set of nonnegative real numbers $\lambda$ such that we can find a Q-basis $e_{1}, \ldots, e_{r}$ of $\mathscr{M}_{\mathrm{Q}}:=$ $\mathscr{M} \otimes_{\mathbb{Z}} \mathbb{Q}$ which is contained in $\mathscr{M}$ (resp. a free basis of $\mathscr{M} / \mathscr{M}_{\text {tor }}$ ) with $\left\|e_{i}\right\| \leq \lambda$ for all $i=1, \ldots, r$. Note that

$$
\lambda_{\mathrm{Q}}(\mathscr{M},\|\cdot\|) \leq \lambda_{\mathbb{Z}}(\mathscr{M},\|\cdot\|) \leq \operatorname{rk}(\mathscr{M}) \lambda_{\mathrm{Q}}(\mathscr{M},\|\cdot\|)
$$

(cf. [14. Lemma 1.2]).

Let $R=\bigoplus_{n=0}^{\infty} R_{n}$ be a graded Q-algebra of finite type such that $R$ is an integral noetherian domain and $\operatorname{dim}_{\mathbb{Q}} R_{n}<\infty$ for all $n \geq 0$. Let $\mathscr{R}=\bigoplus_{n=0}^{\infty} \mathscr{R}_{n}$ be a graded subalgebra of $R$ such that $\mathscr{R}_{n}$ is a finitely generated $\mathbb{Z}$-module and $\mathscr{R}_{n} \otimes_{\mathbb{Z}} \mathbb{Q}=R_{n}$ for all $n \geq 0$. For each $n \geq 0$, let $\|\cdot\|_{n}$ be a norm of $R_{n} \otimes_{\mathbb{Q}} \mathbb{R}(=$ $\left.\mathscr{R}_{n} \otimes_{\mathbb{Z}} \mathbb{R}\right)$. We assume that

$$
(\mathscr{R},\|\cdot\|)=\bigoplus_{n=0}^{\infty}\left(\mathscr{R}_{n},\|\cdot\|_{n}\right)
$$

is a normed graded $\mathbb{Z}$-algebra, that is, for $a \in \mathscr{R}_{n}$ and $b \in \mathscr{R}_{n^{\prime}},\|a \cdot b\|_{n+n^{\prime}} \leq$ $\|a\|_{n} \cdot\|b\|_{n^{\prime}}$.

Let $X:=\operatorname{Proj}(R)$ and $Y$ a closed subvariety of $X$ over $\mathbb{Q}$, that is, $Y$ is a closed, reduced and irreducible subscheme of $X$ over $\mathbb{Q}$. Let $P=\bigoplus_{n=0}^{\infty} P_{n}$ be the corresponding homogeneous prime ideal of $R$ to $Y$. We set

$$
R_{Y, n}:=R_{n} / P_{n}, \mathscr{R}_{Y, n}:=\mathscr{R}_{n} / P_{n} \cap \mathscr{R}_{n}, R_{Y}:=\bigoplus_{n=0}^{\infty} R_{Y, n} \text { and } \mathscr{R}_{Y}:=\bigoplus_{n=0}^{\infty} \mathscr{R}_{Y, n} .
$$

Let $\|\cdot\|_{Y, n}^{\text {quot }}$ be the quotient norm of $R_{Y, n} \otimes_{\mathbb{Q}} \mathbb{R}$ induced by the surjective homomorphism $R_{n} \otimes_{\mathbb{Q}} \mathbb{R} \rightarrow R_{Y, n} \otimes_{\mathbb{Q}} \mathbb{R}$ and the norm $\|\cdot\|_{n}$ on $R_{n} \otimes_{\mathbb{Q}} \mathbb{R}$. Note that $\mathscr{R}_{Y, n} \otimes_{\mathbb{Z}} \mathbb{Q}=R_{Y, n}$ for all $n \geq 0$ and

$$
\left(\mathscr{R}_{Y},\|\cdot\|_{Y}^{\text {quot }}\right)=\bigoplus_{n=0}^{\infty}\left(\mathscr{R}_{Y, n},\|\cdot\|_{Y, n}^{\text {quot }}\right)
$$


is a normed graded $\mathbb{Z}$-algebra. Then we have the following:

Theorem 5.4. Let $\mathfrak{S}_{X}$ be the set of all subvarieties of $X$ and let $v: \mathfrak{S}_{X} \rightarrow \mathbb{R}_{>0}$ be a map. We assume that, for every $Y \in \mathfrak{S}_{X}$, there are a positive integer $n(Y)$ and $s_{Y} \in$ $\mathscr{R}_{Y, n(Y)} \backslash\{0\}$ with $\left\|s_{Y}\right\|_{Y, n(Y)}^{\text {quot }} \leq v(Y)^{n(Y)}$. Then there are a positive number $B$ and $a$ finite subset $S$ of $\mathfrak{S}_{X}$ such that

$$
\lambda_{\mathbb{Q}}\left(\mathscr{R}_{n},\|\cdot\|_{n}\right) \leq B n^{d(d+1) / 2}(\max \{v(Y) \mid Y \in S\})^{n}
$$

for all $n \geq 1$, where $d=\operatorname{dim} X$.

Proof. It is a generalization of [14. Theorem 3.1]. However, it can be proved in the similar way as [14, the proof of Theorem 3.1]. For reader's convenience, we give a sketch of the proof.

Step 1: For a positive integer $h$, we set

$$
R_{n}^{(h)}:=R_{h n}, \quad \mathscr{R}_{n}^{(h)}:=\mathscr{R}_{h n}, \quad R^{(h)}=\bigoplus_{n=0}^{\infty} R_{n}^{(h)} \quad \text { and } \quad \mathscr{R}^{(h)}=\bigoplus_{n=0}^{\infty} \mathscr{R}_{n}^{(h)} .
$$

By using [14, Lemma 2.2 and Lemma 2.4], we can see that if the theorem holds for $\mathscr{R}^{(h)}$ and $v^{h}$, then it holds for $\mathscr{R}$ and $v$. Therefore, by [3, Chapitre III, $\S 1$, Proposition 3], we may assume that $R$ is generated by $R_{1}$ over $R_{0}$ and $s:=s_{X} \in$ $\mathscr{R}_{1}$. Let $\mathscr{O}_{X}(1)$ be the tautological invertible sheaf of $X$ arising from $R_{1}$.

We prove this theorem by induction on $d$.

Step 2: In the case where $d=0, X=\operatorname{Spec}(K)$ for some number field $K$, so that $R_{n} \subseteq H^{0}\left(X, \mathscr{O}_{X}(n)\right) \cong K$. Therefore, $\operatorname{dim}_{\mathbb{Q}} R_{n} \leq[K: \mathbb{Q}]$ for all $n \geq 1$, and hence the assertion can be checked by the same arguments as in [14, Claim 3.1.2].

Step 3: We assume $d>0$. Let $I$ be the homogeneous ideal generated by $s:=$ $s_{X}$, that is, $I=R s$. By using the same ideas as in [12, Chapter I, Proposition 7.4], we can find a sequence

$$
I=I_{0} \subsetneq I_{1} \subsetneq \cdots \subsetneq I_{r}=R
$$

of homogeneous ideals of $R$ and non-zero homogeneous prime ideals $P_{1}, \ldots, P_{r}$ of $R$ such that $P_{i} \cdot I_{i} \subseteq I_{i-1}$ for $i=1, \ldots, r$.

Step 4: We set $\overline{\mathscr{R}}_{n}=\left(\mathscr{R}_{n},\|\cdot\|_{n}\right)$ and $\overline{\mathscr{I}}_{i, n}=\left(\mathscr{I}_{i, n},\|\cdot\|_{i, n}\right)$, where $\mathscr{I}_{i, n}:=\mathscr{R}_{n} \cap$ $I_{i, n}$ and $\|\cdot\|_{i, n}$ is the subnorm induced by $\|\cdot\|_{n}$ and $I_{i, n} \hookrightarrow R_{n}$. Here we consider the following sequence:

$$
\begin{aligned}
& \overline{\mathscr{R}}_{0} \quad \stackrel{\cdot s}{\longrightarrow} \quad \overline{\mathscr{I}}_{0,1} \quad \hookrightarrow \cdots \hookrightarrow \quad \overline{\mathscr{I}}_{i, 1} \quad \hookrightarrow \cdots \hookrightarrow \quad \overline{\mathscr{I}}_{r, 1}=\overline{\mathscr{R}}_{1} \\
& \begin{array}{cccccc}
\vdots & \vdots & \vdots & \vdots & \vdots & \vdots \\
\stackrel{s}{\longrightarrow} & \overline{\mathscr{I}}_{0, j} & \hookrightarrow \cdots \hookrightarrow & \overline{\mathscr{I}}_{i, j} & \hookrightarrow \cdots \hookrightarrow & \overline{\mathscr{I}}_{r, j}=\overline{\mathscr{R}}_{j}
\end{array} \\
& \stackrel{\cdot s}{\longrightarrow} \overline{\mathscr{I}}_{0, j+1} \quad \hookrightarrow \cdots \hookrightarrow \overline{\mathscr{I}}_{i, j+1} \quad \hookrightarrow \cdots \hookrightarrow \overline{\mathscr{I}}_{r, j+1}=\overline{\mathscr{R}}_{j+1} \\
& \begin{array}{cccccc}
\vdots & \vdots & \vdots & \vdots & \vdots & \vdots \\
\stackrel{\cdot s}{\longrightarrow} & \overline{\mathscr{I}}_{0, n} & \hookrightarrow \cdots \hookrightarrow & \overline{\mathscr{I}}_{i, n} & \hookrightarrow \cdots \hookrightarrow & \overline{\mathscr{I}}_{r, n}=\overline{\mathscr{R}}_{n}
\end{array}
\end{aligned}
$$

Let $\|\cdot\|_{i, n}^{\text {quot }}$ be the quotient norm of $I_{i, n} / I_{i-1, n}$ induced by $\|\cdot\|_{i, n}$ and $I_{i, n} \rightarrow I_{i, n} / I_{i-1, n}$. Note that $\mathscr{I}_{0, n} / \mathscr{R}_{n-1} S$ is a torsion module for all $n \geq 1$, so that, applying [14. 
Proposition 1.4] to the above sequence, we have

$$
\begin{aligned}
\lambda_{\mathbb{Q}}\left(\overline{\mathscr{R}}_{n}\right) \leq \sum_{j=1}^{n}\left(\sum_{i=1}^{r}\|s\|_{1}^{n-i} \lambda_{\mathbb{Q}}\left(\mathscr{I}_{i, j} / \mathscr{I}_{i-1, j}\|\cdot\|_{i, j}^{\text {quot }}\right)\right. & \left.\operatorname{dim}_{\mathbf{Q}}\left(I_{i, j} / I_{i-1, j}\right)\right) \\
& +\|s\|_{1}^{n} \lambda_{\mathbb{Q}}\left(\overline{\mathscr{R}}_{0}\right) \operatorname{dim}_{\mathbb{Q}} R_{0} .
\end{aligned}
$$

Step 5: Here we claim the following:

Claim 5.4.1. (1) If $P_{i} \in \operatorname{Proj}(R)$, then there are positive constants $B_{i}$ and $C_{i}$, and $a$ finite subset $S_{i}$ of $\mathfrak{S}_{X}$ such that

$$
\lambda_{\mathrm{Q}}\left(\mathscr{I}_{i, n} / \mathscr{I}_{i-1, n},\|\cdot\|_{i, n}^{\text {quot }}\right) \leq B_{i} n^{d(d-1) / 2}\left(\max \left\{v(Y) \mid Y \in S_{i}\right\}\right)^{n}
$$

and $\operatorname{dim}_{Q}\left(I_{i, n} / I_{i-1, n}\right) \leq C_{i} n^{d-1}$ for all $n \geq 1$.

(2) If $P_{i} \notin \operatorname{Proj}(R)$, then there is a positive integer $n_{i}$ such that $I_{i, n} / I_{i-1, n}=0$ for $n \geq n_{i}$. In particular, $\lambda_{\mathrm{Q}}\left(\mathscr{I}_{i, n} / \mathscr{I}_{i-1, n},\|\cdot\|_{i, n}^{\text {quot }}\right)=0$ and $\operatorname{dim}_{\mathrm{Q}}\left(I_{i, n} / I_{i-1, n}\right)=$ 0 for all $n \geq n_{i}$.

Proof. (1) follows from [14, Proposition 2.3] and the hypothesis of induction. In the case (2), $P_{i}=\bigoplus_{n=1}^{\infty} R_{n}$ because $R_{0}$ is a number field. As $I_{i} / I_{i-1}$ is a finitely generated $\left(R / P_{i}\right)$-module, we can find a positive integer $n_{i}$ such that $I_{i, n} / I_{i-1, n}=$ 0 for $n \geq n_{i}$.

Step 6: The assertion of the theorem follows from (5.3) by using (1) and (2) of Claim 5.4.1

5.3. Nakai-Moishezon's criterion. Let $X$ be a geometrically integral projective variety over a number field $K$. For a closed subvariety $Y$ of $X$ and $v \in M_{K}$, we set $Y_{v}:=Y \times_{\operatorname{Spec}(K)} \operatorname{Spec}\left(K_{v}\right)$. Let $L$ be an invertible sheaf on $X$. For $v \in M_{K}$, let $h_{v}$ be a continuous metric of $L_{v}^{\text {an }}$ on $X_{v}^{\text {an }}$, where $L_{v}:=L \otimes_{K} K_{v}$. Note that $X(\mathbb{C})$ is canonically identified with $\bigsqcup_{\sigma \in M_{K}^{\infty}} X_{\sigma}(\mathbb{C})$, so that $h_{\infty}:=\left\{h_{\sigma}\right\}_{\sigma \in M_{K}^{\infty}}$ yields a metric on $L_{\infty}$. We assume that $h_{\infty}$ is invariant by the complex conjugation map $F_{\infty}$ on $X(\mathbb{C})$. Moreover, for $s \in H^{0}\left(Y,\left.L\right|_{Y}\right)$, we set

$$
\|s\|_{Y_{v}, h_{v}}:=\sup \left\{|s|_{h_{v}}(x) \mid x \in Y_{v}^{\mathrm{an}}\right\}
$$

Theorem 5.5. We assume the following:

(a) For any $n \in \mathbb{Z}_{\geq 0},\left(H^{0}\left(X, L^{\otimes n}\right),\left\{\|\cdot\|_{X_{v}, h_{v}^{n}}\right\}_{v \in M_{K}}\right)$ is an adelically normed vector space over $K$.

(b) $\left.\bar{L}\right|_{Y}$ is big for all subvarieties $Y$ of $X$, that is, $\left.L\right|_{Y}$ is big on $Y$ and there are a positive integer $n$ and $s \in H^{0}\left(Y,\left.L\right|_{Y} ^{\otimes n}\right) \backslash\{0\}$ such that $\|s\|_{Y_{p}, h_{p}^{n}} \leq 1$ for all $\mathfrak{p} \in M_{K}^{\text {fin }}$ and $\|s\|_{Y_{\sigma}, h_{\sigma}^{n}}<1$ for all $\sigma \in M_{K}^{\infty}$.

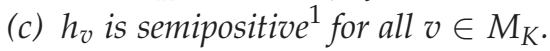

Then there are positive numbers $B$ and $v$ such that $v<1$ and

$$
\lambda_{\mathrm{Q}}\left(\left(H^{0}\left(X, L^{\otimes n}\right),\|\cdot\|_{h^{n}}\right)_{\leq 1}^{\text {fin }}, \max _{\sigma \in M_{K}^{\infty}}\left\{\|\cdot\|_{X_{\sigma}, h_{\sigma}^{n}}\right\}\right) \leq B n^{d(d+1) / 2} v^{n}
$$

\footnotetext{
${ }^{1}$ In the case where $v \in M_{K}^{\infty}$, the semipositivity of $h_{v}$ can be defined as the uniform limit of the quotient metrics as described in 3.3 This semipositivity coincides with the positivity of the first Chern current of $\left(L_{v}, h_{v}\right)$. For details, see [16].
} 
for all $n \geq 1$.

Proof. First note that $L$ is nef because $\left.L\right|_{C}$ is big for all curves $C$ on $X$. Moreover, as $\left.L\right|_{Y}$ is big on $Y$ and $L$ is nef, we have $\left(\left.L\right|_{Y} ^{\operatorname{dim} Y}\right)>0$. Therefore, $L$ is ample on $X$ by virtue of the Nakai-Moishezon criterion for projective algebraic varieties.

We set

$$
R_{n}:=H^{0}\left(X, L^{\otimes n}\right), \quad \mathscr{R}_{n}:=\left(R_{n},\|\cdot\|_{h^{n}}\right)_{\leq 1}^{\mathrm{fin}}, \quad\|\cdot\|_{n}:=\max _{\sigma \in M_{K}^{\infty}}\left\{\|\cdot\|_{X_{\sigma}, h_{\sigma}^{n}}\right\} .
$$

Note that $\mathscr{R}_{n}$ is a finitely generated $\mathbb{Z}$-module by (2) in Lemma 5.2 We use the same notation as in Section 5.2 Note that $X=\operatorname{Proj}(R)$ because $L$ is ample. Fix a closed subvariety $Y$. For $v \in M_{K}$, the norm $\|\cdot\|_{X_{v}, h_{v}^{n}}$ on $H^{0}\left(X_{v}, L_{v}^{\otimes n}\right)$ (resp. the norm $\|\cdot\|_{Y_{v}, h_{v}^{n}}$ on $\left.H^{0}\left(Y_{v},\left.L\right|_{Y_{v}} ^{\otimes n}\right)\right)$ is denoted by $\|\cdot\|_{X_{v}, n}$ (resp. $\left.\|\cdot\|_{Y_{v}, n}\right)$. Note that $\|\cdot\|_{n}=\max _{\sigma \in M_{K}^{\infty}}\left\{\|\cdot\|_{X_{\sigma}, n}\right\}$. Let $\|\cdot\|_{Y_{v}, n}^{\text {quot }}$ be the quotient norm of $R_{Y, n} \otimes_{K}$ $K_{v}$ induced by $\|\cdot\|_{X_{v}, n}$ and the surjective homomorphism $R_{n} \otimes_{K} K_{v} \rightarrow R_{Y, n} \otimes_{K}$ $K_{v}$. We also fix a positive integer $n_{0}$ such that, for all $n \geq n_{0}, H^{0}\left(X, L^{\otimes n}\right) \rightarrow$ $H^{0}\left(Y,\left.L\right|_{Y} ^{\otimes n}\right)$ is surjective.

By (3) in Lemma 5.2 and Theorem 5.4, it is sufficient to show that there are a positive integer $n(Y) \geq n_{0}$ and $s_{Y} \in H^{0}\left(Y,\left.L\right|_{Y} ^{\otimes n(Y)}\right) \backslash\{0\}$ such that $\|s\|_{Y_{p}, n(Y)}^{\text {quot }} \leq 1$ for all $\mathfrak{p} \in M_{K}^{\text {fin }}$ and $\|s\|_{Y_{\sigma, n(Y)}}^{\text {quot }}<1$ for all $\sigma \in M_{K}^{\infty}$.

As $\left.\bar{L}\right|_{Y}$ is big, there are $n_{1}>0$ and $s^{\prime} \in H^{0}\left(Y,\left.L\right|_{Y} ^{\otimes n_{1}}\right)$ such that $\left.\left\|s^{\prime}\right\|\right|_{Y_{\mathfrak{p}}, n_{1}} \leq 1$ for all $\mathfrak{p} \in M_{K}^{\text {fin }}$ and $\left\|s^{\prime}\right\|_{Y_{\sigma}, n_{1}}<1$ for all $\sigma \in M_{K}^{\infty}$. Since $H^{0}\left(X, L^{\otimes n_{0} n_{1}}\right) \rightarrow$ $H^{0}\left(Y,\left.L\right|_{Y} ^{\otimes n_{0} n_{1}}\right)$ is surjective, we can find $l^{\prime} \in H^{0}\left(X, L^{\otimes n_{0} n_{1}}\right)$ such that $\left.l^{\prime}\right|_{Y}=s^{\prime \otimes n_{0}}$, so that there are $\mathfrak{p}_{1}, \ldots, \mathfrak{p}_{e} \in M_{K}^{\text {fin }}$ such that $\left\|l^{\prime}\right\|_{X_{\mathfrak{p}}, n_{0} n_{1}} \leq 1$ for all $\mathfrak{p} \in M_{K}^{\text {fin }} \backslash$

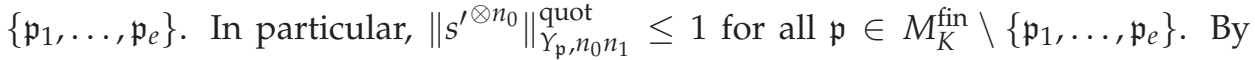
Lemma 5.3, we can choose $\beta \in \mathcal{O}_{K} \backslash\{0\}$ such that

$$
\operatorname{ord}_{v}(\beta) \begin{cases}>0 & \text { if } v \in\left\{\mathfrak{p}_{1}, \ldots, \mathfrak{p}_{e}\right\}, \\ =0 & \text { if } v \in M_{K} \backslash\left\{\mathfrak{p}_{1}, \ldots, \mathfrak{p}_{e}\right\} .\end{cases}
$$

Since $\|s\|_{Y_{\sigma}, n_{1}}<1$ for all $\sigma \in M_{K}^{\infty}$, we can find a positive integer $n_{2}$ such that

$$
\left(\max _{\sigma \in M_{K}^{\infty}}\left\{\|s\|_{Y_{\sigma}, n_{1}}\right\}\right)^{n_{0} n_{2}} \max \left\{|\sigma(\beta)| \mid \sigma \in M_{K}^{\infty}\right\}<1 .
$$

Claim 5.5.1. If we set $s=\beta s^{\prime}{ }^{\otimes n_{0} n_{2}}$, then s satisfies the following properties:

(i) $\|s\|_{Y_{\mathfrak{p}}, n_{2} n_{1} n_{0}}^{\text {quot }} \leq 1$ for all $\mathfrak{p} \in M_{K}^{\text {fin }} \backslash\left\{\mathfrak{p}_{1}, \ldots, \mathfrak{p}_{e}\right\}$.

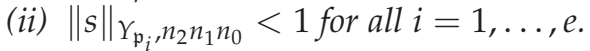

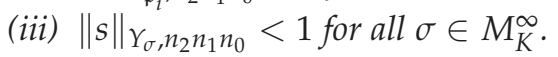

Proof. (i) is obvious. (iii) follows from (5.4). Let us consider (ii). As $\operatorname{ord}_{\mathfrak{p}_{i}}(\beta)>0$ and $\left\|s^{\prime}\right\|_{\mathfrak{p}_{i}, n_{1}} \leq 1$, we have

$$
\begin{aligned}
& \|s\|_{Y_{\mathfrak{p}_{i},}, n_{2} n_{1} n_{0}}=\#\left(\mathcal{O}_{K} / \mathfrak{p}_{i}\right)^{-\operatorname{ord}_{\mathfrak{p}_{i}}(\beta)}\left\|s^{\prime \otimes n_{0} n_{2}}\right\|_{Y_{\mathfrak{p}_{i}, n_{0} n_{1} n_{2}}} \\
& =\#\left(\mathcal{O}_{K} / \mathfrak{p}_{i}\right)^{-\operatorname{ord}_{\mathfrak{p}_{i}}(\beta)}\left(\left\|s^{\prime}\right\|_{{\mathfrak{p _ { i }}}_{i}, n_{1}}\right)^{n_{0} n_{2}}<1 \text {, }
\end{aligned}
$$

as required. 
Next let us see the following claim:

Claim 5.5.2. If $\|t\|_{Y_{v}, m}<1$ for $v \in M_{K}$ and $t \in H^{0}\left(Y_{v},\left.L_{v}\right|_{Y_{v}} ^{\otimes m}\right)$, then there is $m_{0}$ such that, for all $m^{\prime} \geq m_{0}$,

$$
\left\|t^{\otimes m^{\prime}}\right\|_{Y_{v}, m m^{\prime}}^{\text {quot }}<1 \text {. }
$$

Proof. Choose $\epsilon>0$ such that $e^{\epsilon}\|t\|_{Y_{v}, m}<1$. By virtue of the extension property (cf. [16] and Theorem 4.2), there is $m_{0}$ such that, for all $m^{\prime} \geq m_{0}$, we can find $t^{\prime} \in$ $H^{0}\left(X_{v}, L_{v}^{\otimes m m^{\prime}}\right)$ with $\left.t^{\prime}\right|_{Y_{v}}=t^{\otimes m^{\prime}}$ and $\left\|t^{\prime}\right\|_{X_{v}, m m^{\prime}} \leq e^{m^{\prime} \epsilon}\left(\|t\|_{Y_{v}, m}\right)^{m^{\prime}}$. In particular, $\left\|t^{\prime}\right\|_{X_{v}, m m^{\prime}}<1$, so that the assertion follows.

By the above claim, for each $i=1, \ldots, e$ and $\sigma \in M_{K}^{\infty}$, there is a positive integer $n_{3}$ such that

$$
\left\|s^{\otimes n_{3}}\right\|_{Y_{\mathfrak{p}_{i}, n_{3} n_{2} n_{1} n_{0}}^{\text {quot }}}^{\text {qu }}<1 \text { and }\left\|s^{\otimes n_{3}}\right\|_{Y_{\sigma}, n_{3} n_{2} n_{1} n_{0}}^{\text {quot }}<1 \text {. }
$$

We set $n(Y):=n_{3} n_{2} n_{1} n_{0}$ and $s_{Y}:=s^{\otimes n_{3}}$, then $\left\|s_{Y}\right\|_{Y_{p}, n(Y)}^{\text {quot }} \leq 1$ for all $\mathfrak{p} \in M_{K}^{\text {fin }}$ and $\left\|s_{Y}\right\|_{Y_{\sigma, n(Y)}}^{\text {quot }}<1$ for all $\sigma \in M_{K}^{\infty}$.

Corollary 5.6. We assume (a), (b) and (c) in Theorem 5.5 Let $(N, g)$ be a pair of an invertible sheaf $N$ on $X$ and a family $g=\left\{g_{v}\right\}_{v \in M_{K}}$ of continuous metrics $g_{v}$ of $N_{v}^{\text {an }}$ on $X_{v}^{\text {an }}$. We assume that $g_{\infty}:=\left\{g_{\sigma}\right\}_{\sigma \in M_{K}^{\infty}}$ is compatible with respect to $F_{\infty}$ and

$$
\left(H^{0}\left(X, L^{\otimes n} \otimes N\right),\left\{\|\cdot\|_{X_{v}, h_{v}^{n} g_{v}}\right\}_{v \in M_{K}}\right)
$$

is an adelically normed vector space over $K$ for all $n \geq 0$. Then there is a positive integer $n_{0}$ such that, for $n \geq n_{0},\left(H^{0}\left(X, L^{\otimes n} \otimes N\right),\|\cdot\|_{h^{n} g}\right)_{\leq 1}^{\text {fin }}$ has a free basis $\left(\omega_{1}, \ldots, \omega_{r_{n}}\right)$ over $\mathbb{Z}$ with $\left\|\omega_{i}\right\|_{h_{\sigma}^{n} g_{\sigma}}<1$ for all $i=1, \ldots, r_{n}$ and $\sigma \in M_{K}^{\infty}$, where $r_{n}$ is the rank of $H^{0}\left(X, L^{\otimes n} \otimes N\right)$ over $\mathbf{Q}$.

Proof. We use the same notation in the proof of Theorem5.5 Moreover, we set

$$
\left\{\begin{array}{l}
A_{n}:=H^{0}\left(X, L^{\otimes n} \otimes N\right), \\
\mathscr{A}_{n}:=\left(H^{0}\left(X, L^{\otimes n} \otimes N\right),\|\cdot\|_{h^{n} g}\right)_{\leq 1}^{\text {fin }}, \quad\|\cdot\|_{n}^{\prime}:=\max _{\sigma \in M_{K}^{\infty}}\left\{\|\cdot\|_{X_{\sigma}, h_{\sigma}^{n} g_{\sigma}}\right\}_{\sigma \in M_{K}^{\infty},} \\
A:=\bigoplus_{n=0}^{\infty} A_{n}, \\
\left(\mathscr{A},\|\cdot\|^{\prime}\right):=\bigoplus_{n=0}^{\infty}\left(\mathscr{A}_{n},\|\cdot\|_{n}^{\prime}\right) .
\end{array}\right.
$$

Note that $\left(\mathscr{A},\|\cdot\|^{\prime}\right)$ is a normed graded $(\mathscr{R},\|\cdot\|)$-module (cf. [14, Section 2]). Further $A$ is a finitely generated over $R$ because $L$ is ample. Therefore, by Theorem 5.5 together with [14, Lemma 2.2], there is a positive number $B^{\prime}$ such that $\lambda_{\mathrm{Q}}\left(\mathscr{A}_{n},\|\cdot\|_{n}^{\prime}\right) \leq B^{\prime} n^{d(d+1) / 2} v^{n}$ for all $n \geq 1$, so that, by (5.2),

$$
\lambda_{\mathbb{Z}}\left(\mathscr{A}_{n},\|\cdot\|_{n}^{\prime}\right) \leq \operatorname{dim}_{\mathbb{Q}} H^{0}\left(X, L^{\otimes n} \otimes N\right) \cdot B^{\prime} n^{d(d+1) / 2} v^{n}
$$

for all $n \geq 1$. Thus we can find a positive integer $n_{0}$ such that $\lambda_{\mathbb{Z}}\left(\mathscr{A}_{n},\|\cdot\|_{n}^{\prime}\right)<1$ for $n \geq n_{0}$, as required. 


\section{REFERENCES}

[1] M. F. Atiyah and I. G. Macdonald, Introduction to Commutative Algebra, Addison-Wesley (1969).

[2] V. G. Berkovich, Spectral theory and analytic geometry over non-Archimedean fields, Mathematical surveys and monographs, No. 33, American Mathematical Society, Providence, RI, 1990.

[3] N. Bourbaki, Éléments de Mathématique, Algèbre commutative, Chapitres 1 à 4, Masson, Paris, 1985.

[4] N. Bourbaki, Éléments de Mathématique, Espaces vectoriel topologique, Chapitres 1 à 5, Masson, Paris, 1981.

[5] S. Boucksom and H. Chen, Okounkov bodies of filtered linear series, Compositio Math. 147 (2011), 1205-1229.

[6] J. I. Burgos Gil, A. Moriwaki, P. Philippon and M. Sombra, Arithmetic positivity on toric varieties, to appear in Journal of Algebraic Geometry, (see also arXiv:1210.7692 1 [math.AG]).

[7] A. Chambert-Loir and A. Ducros, Formes différentielles réelles et courants sur les espaces de Berkovich, arXiv:1204.6277

[8] J.-P. Demailly, On the Ohsawa-Takegoshi-Manivel $L^{2}$ extension theorem, in Complex Analysis and Geometry (Paris, 1997), Progress in Mathematics 188, 47-82.

[9] W. Gubler, Local heights of subvarieties over non-Archimedean fields, Journal für die Reine und Angewandte Mathematik, 498 (1998), 61-113.

[10] W. Gubler, A guide to tropicalizations, in Algebraic and Combinatorial Aspects of Tropical Geometry, Contemporary Mathematics, Vol. 589, American Mathematical Society, Providence, RI, 2013, pp. $125-189$.

[11] W. Gubler and K. Künnemann, Positivity properties of metrics and delta-forms, arXiv:1509.09079

[12] R. Hartshorne, Algebraic geometry, Graduate Texts in Mathematics, No. 52. Springer, New York, (1977).

[13] A. Moriwaki, Estimation of arithmetic linear series, Kyoto Journal of Mathematics 50 (Memorial issue of Professor Nagata) (2010), 685-725.

[14] A. Moriwaki, Free basis consisting of strictly small sections, International Mathematics Research Notices (2011), 1245-1267.

[15] A. Moriwaki, Adelic divisors on arithmetic varieties, to appear in Memoirs of the American Mathematical Society, see also preprint arXiv:1302.1922 [math.AG]).

[16] A. Moriwaki, Semiample invertible sheaves with semipositive continuous hermitian metrics, Algebra \& Number Theory 9 (2015), 503-509.

[17] C. Perez-Garcia and W. H. Schikhof, Locally convex spaces over non-Archimedean valued fields, Cambdridge Studies in Advanced Mathematics, vol.119, Cambridge University Press, 2010.

[18] H. Randriambololona, Métriques de sous-quotient et théorème de Hilbert-Samuel arithmétique pour les faisceaux cohérents, Journal für die Reine und Angewandte Mathematik 590 (2006), 67-88.

[19] G. Tian, On a set of polarized Kähler metrics on algebraic manifolds, Journal of Differential Geometry 32 (1990), no.1, 99-130.

[20] S. Zhang, Positive line bundles on arithmetic varieties, Journal of the American Mathematical Society 8 (1995), 187-221.

E-mail address: huayi.chen@ujf-grenoble.fr

Institut Fourier, Université Grenoble Alpes, 100 RUe des Mathématiques, 38402 SaintMartin-d'Hères Cedex

E-mail address: moriwaki@math.kyoto-u.ac.jp

Department of Mathematics, Faculty of Science, Kyoto University, Kyoto, 606-8502, Japan 